Chapter 6

\title{
Interactive Maps on Variant Phase Spaces \\ - From Measurements - Micro Ensembles to Ensemble Matrices on Statistical Mechanics of Particle Models
}

Jeffrey Zheng, Christian Zheng and Tosiyasu Kunii

Additional information is available at the end of the chapter

http://dx.doi.org/10.5772/51635

\section{Introduction}

\subsection{Fundamental models of cellular automata and phase space}

\subsubsection{White and Black Box Models}

Input, output and functions are fundamental elements of the wider applications of dynamic systems [3, 5, 21] such applications include: mathematics, probability, physics, statistics, classical logic, and cellular automata.

For a pair of $N$ bit vectors $X, Y \in B_{2}^{N}$ with states, for a given $0-1$ function $f$, the pair of $0-1$ vectors are linked by an equation where the function may be expressed by $Y=f(X)$ thus:

$$
\text { Input } X \rightarrow \begin{gathered}
- \text { White Box - } \\
\text { Given function } f
\end{gathered} \rightarrow \text { Output } Y \text {. }
$$

This is called a white box model $[3,15,28]$. Using the white box model, a pair $(X, Y)$ can be explicitly calculated by a function $f$.

If there is no explicit expression for a unknown function $U$, a pair of vectors $(X, Y)$ could be collected for their correspondences on the pair of input-output relationships. Equation $Y=U(X)$ is still satisfied. This is called a black box model. i.e. A pair of $(X, Y)$ can be measured by a unknown function $U$, or expressed as 


$$
\text { Input } X \rightarrow \begin{gathered}
- \text { Black Box - } \\
\text { Unknow function } U
\end{gathered} \rightarrow \text { Output } Y \text {. }
$$

In science and engineering [13, 28, 29], a black box is a device, system, or object which can be viewed solely in terms of its input, output and transfer characteristics without any knowledge of its internal works.

From a cellular automata viewpoint, the black box approach is useful in describing a situation where both input and output are in the form of two bit vectors for an unknown function of a digital system.

\subsubsection{Characteristic Point and Phase Space}

In mathematics and physics [3, 14, 18, 20, 21, 29], the concept of a phase space as introduced by W. Gibbs in 1901 is a space in which all possible states of a system are represented. Here, each possible state of the system corresponds to one unique point in the phase space. For cellular automata, the phase space usually consists of all possible values of pairs of input and output vectors in multiple dimensions.

For either a known function $f$ or for an unknown function $U$, when the states of $X, Y$ reside in the same finite region, it is entirely feasible in principle to undertake an exhaustive procedure to list all pairs of $\{(X, Y)\}$. For a given $N$ bit vector $X$, the vector generates a point with a unique spatial position to indicate the characteristics of the function and by listing all such possible points, a phase space for the function is established.

\subsection{Historical review on phase spaces of statistical mechanics}

Top-down and bottom-up are two distinct strategies of intelligent processing and knowledge ordering used in humanistic and scientific theories $[4,13,15,25,28]$. In practice, they can be seen as alternative styles of thinking and problem solving. Top-down may be taken to mean an approach based on an analysis or decomposition to identify key components within a global target that has been identified for study and from which there may be constructed a hierarchy of local features. Bottom-up may be taken to describe a process of synthesis via integration working from local features towards a global target.

\subsubsection{Bottom-up Approaches}

Isaac Newton (1642-1727) and Gottfried Wilhelm Leibniz (1646-1716) gave calculus to the world of mathematics during the decade 1670-1680. This established a systematic methodology for the efficient analysis of local variables in order to reveal global features.

Joseph-Louis Lagrange (1736-1813) took the conservation of energy as the foundation for his system of mechanics where he combined the principle of virtual velocities with the principle of least action. Along these lines, W. R. Hamilton (1805-65) established his approach to dynamics in 1834-1835 with the first description of functions on phase space with pairs of conjugate parameters, together with position and momentum. J. Liouville (1809-82) proposed a theorem on the conservation of volume in phase space in 1838. C. G. Jacobi 
(1804-51) recognized that Liouville's works could be used to describe mechanical systems and so placed Liouville's mathematical theorem into a mechanical context. Pücker working in Germany and Cayley and Sylvester in the UK, extended projective geometry beyond the ordinary three dimensions in the 1840s and Grassmann developed an $n$ dimensional vector space in 1844 .

Riemannn's work in 1868 developed the geometric properties of multi-dimensional manifolds. This was followed by further developments in the 1870 s by E. Betti, F. Klein, and $\mathrm{C}$. Jordan then more recently by [23, 24, 31, 33].

As it was Lagrange who took the first steps, a bottom-up approach is now often described as a Lagrange expression. Hamiltonian dynamics is a typical representative under this expression as it is founded on a pair of conjugate parameters [31,33].

\subsubsection{Top-down Approaches}

Robert Boyle (1627-91) developed new physio-mechanical experiments. Boyle's law states that at a constant temperature, the volume of a fixed mass of gas is inversely proportional to its pressure using a set of measures characterizing the global properties of a gas. Anders Celsius (1701-1744) proposed a thermometer scale calibrated to the freezing point and boiling point of water. Benjamin Thompson (later known as Count Rumford) (1753-1814) explored cannon barrel-boring experiments and demonstrated the conversion of work into heat via friction in the absence of any additional weight of the object due to such heating being detected. Leonhard Euler (1707-1783) developed a Kinetic Heat Theory based on his description of a calculus of variations to introduce the concept of moving axes in astronomy. Daniel Bernoulli (1700-1782) and Pierre-Simon Laplace (1749-1827) refined Newton's work to represent gas properties through repulsive interactions. Jean Baptiste Joseph Fourier (1768-1830) developed an understanding of the conduction of heat to represent a periodic function as a Fourier series. Poisson (1781-1840) further developed the theories of heat using Fourier series. Thomas Young (1773-1829) expressed the modern formulation of energy, mathematically associated with $m v^{2}$. Sadi Carnot (1796-1832) introduced the concept of ideal gas cycle analysis. William Thomson (later known as Lord Kelvin) (1824-1907) developed a wave theory of heat in homogeneous solid bodies. James Prescott Joule (1818-1889) established the relationship between heat and mechanical work through a series of experiments. John James Waterston (1811-1883) explored a kinetic theory of gases and mean free path. Von Helmholtz (1821-94) further developed the principle of conservation of energy extending Carnot's principle of kinetic energy into a mathematical formulation. Rudolf Clausius (1822-88) explored an expression of the second law for which the only function is the transfer of heat to propose the function $d Q / T$ to compare heat flows with heat conversions using Carnot's techniques to derive entropy and show the two laws of thermodynamics were the equivalent of the older caloric theory. Gustav Robert Kirchhoff (1824-1887) derived from the second law of thermodynamics that objects cannot be distinguished by thermal radiation at a uniform temperature to formulate a black body $[23,24,31,33]$.

Leonhard Euler (1707-1783) provided key methodologies in this direction with a top-down approach known as a Euler expression. A Fourier series of a periodic function is a typical representative under this expression founded on a periodic function composed of a set of simple harmonic components [31,33]. 


\subsubsection{Formal Expression of Phase Space in Statistical Mechanics}

Following methodologies established by Hamilton, Lagrange, and Euler, [26] and L. Boltzmann (1844-1906) went on to lay the foundations of statistical mechanics from 1871. They introduced the term phase to describe the analogy they saw between the physical trajectories of particles in two dimensional space and Lissajous figures expressed as interactive maps. When two harmonic frequencies exist as rational fractions, period 4 circular patterns occur. However, when the frequency ratio is irrational, the system trajectories visit all points on the plane bounded by the signal amplitude. J. C. Maxwell (1831-79) adopted Boltzmann's expression of phase to describe the state of systems in 1879. William Thomson (Lord Kelvin) was the first to use the word demon for Maxwell's Thermodynamics concept in 1874. H. Poincarè (1854-1912) in 1885 took a geometric approach to visualize a saddle point where stable and unstable trajectories intersected in phase space. Various mapping techniques are relevant to such explorations. These include Poincarè sections (maps), fixed-point classifications, and the conservation of phase space as an integrated invariant. Influenced by the work of Maxwell and Boltzmann together with other wider contributions, J. W. Gibbs (1939-1903) proposed his Elementary Principles in Statistical Mechanics in 1902 to describe a phase as represented by a point of $2 n$ dimensions.

From a terminological viewpoint, Gibbs brought us such expressions as statistical mechanics, micro ensemble, canonical ensemble, and grand ensemble. He facilitated the establishment of a hierarchy in Statistical Mechanics. However, Gibbs did not use the term phase space. The first formal expression of the term phase space appeared in the context of ergodic theory in a 1913 publication by A. Rosenthal and M. Plancherel [26, 31, 33].

From 1919 to 1922, Sir Charles Galton Darwin (1887-1962) worked with Sir Ralph Howard Fowler (1889-1944) on statistical mechanics and established the Darwin-Fowler method.

\subsubsection{Key Properties in Statistical Mechanics}

$[23,24,31,33]$ noted the usefulness of listing key properties in classical statistical mechanics. A typical comparison is presented below in Table 1.

In general, both Maxwell-Boltzmann and Gibbs follow black-box models without involving explicit local functions. However, the Darwin-Fowler method uses a complex function to describe its unit cell so making it a white box model. Both Maxwell-Boltzmann and Darwin-Fowler schemes use Lagrange expressions to calculate cell unit and to form their fundamentals using a bottom-up strategy. Meanwhile, Gibbs applies Euler expressions for analysis using a top-down strategy without involving explicit cell units.

Table 1 uses abbreviations as follows: TM for Time Measurement, PSM for Phase Space Measurement, PS for Phase Space, EPV for Equal Phase Volume, MPD for Most Probable Distribution, MCE for Micro Canonical Ensemble, CE for Canonical Ensemble, and GCE for Grand Canonical Ensemble.

\subsubsection{Common Interpretations of Quantum Mechanics}

Quantum mechanics is a modern legacy with its roots in classical statistical mechanics [11, 12, 17]. Meanwhile, Bose-Einstein, Fermi-Dirac statistics, and Planck's quantum are deeply connected with the statistical mechanics of Boltzmann and Gibbs [17, 19]. 


\begin{tabular}{|c|c|c|c|}
\hline $\begin{array}{l}\text { Key } \\
\text { Issue }\end{array}$ & $\begin{array}{l}\text { Maxwell-Boltzmann } \\
\text { Most probable theory }\end{array}$ & $\begin{array}{l}\text { Darwin-Fowler } \\
\text { Average Theory }\end{array}$ & $\begin{array}{c}\text { Gibbs } \\
\text { Ensemble Theory }\end{array}$ \\
\hline Assumption & $\begin{array}{c}\text { Ergodic Average: } \\
\text { TM = PSM }\end{array}$ & $\begin{array}{c}\text { Ergodic Average: } \\
\text { TM = PSM }\end{array}$ & $\begin{array}{c}\text { Equality of PS: } \\
\text { EPV in same probability }\end{array}$ \\
\hline $\begin{array}{c}\text { Phase Space } \\
\text { N }\end{array}$ & $\begin{array}{c}\text { State } \\
\text { combination }\end{array}$ & $\begin{array}{c}\text { State } \\
\text { combination }\end{array}$ & $\begin{array}{l}\text { Density functions } \\
\text { Liouville equation }\end{array}$ \\
\hline $\begin{array}{l}\text { Cell Unit } \\
\mathrm{n}\end{array}$ & $\begin{array}{l}\text { Local cell in } \mathrm{n} \text { particles } \\
\text { Stirling Approximation }\end{array}$ & $\begin{array}{l}\text { Complex function } \\
\text { non restriction to } \mathrm{n}\end{array}$ & $\begin{array}{l}\text { Ensemble based } \\
\text { non-cell required }\end{array}$ \\
\hline $\begin{array}{c}\text { Balanced } \\
\text { States }\end{array}$ & $\begin{array}{c}\text { MPD } \\
\text { with maximal entropy }\end{array}$ & $\begin{array}{c}\text { MPD } \\
\text { with maximal entropy }\end{array}$ & $\begin{array}{c}\text { MPD } \\
\text { with maximal entropy }\end{array}$ \\
\hline Expression & Lagrange & Lagrange & Euler \\
\hline Interaction & No & No & Yes \\
\hline $\begin{array}{l}\text { Prefer } \\
\text { System }\end{array}$ & $\begin{array}{l}\text { Isolated system } \\
\text { for }\{\mathrm{MCE}, \mathrm{CE}\} \\
\text { not for GCE }\end{array}$ & $\begin{array}{l}\text { Isolated system } \\
\text { for }\{\mathrm{MCE}, \mathrm{CE}\} \\
\text { not for GCE }\end{array}$ & $\begin{array}{l}\text { MCE: isolated system; } \\
\text { CE : closed system; } \\
\text { GCE : open system }\end{array}$ \\
\hline Model & $\begin{array}{c}\text { Black-box } \\
\text { non function } \\
\text { Bottom-up }\end{array}$ & $\begin{array}{c}\text { White-box } \\
\text { explicit function } \\
\text { Bottom-up }\end{array}$ & $\begin{array}{l}\text { Black-box } \\
\text { non function } \\
\text { Top-down }\end{array}$ \\
\hline
\end{tabular}

Table 1. Key Methods in Statistical Mechanics

In the context of the pursuit of an interpretation of quantum mechanics, the state vector or wave function has been widely discussed as a model for describing the individual components of a system (e.g. an electron).

The most comprehensive descriptions of an individual physical system are to be found in the various versions of the Copenhagen interpretation [8], or in subsequent versions incorporating minor modifications as in the hidden variable interpretations $[19,31]$.

An interpretation according to the state vector based not on an individual system but on an ensemble of identically prepared systems is known as a statistical ensemble interpretation or more briefly just as a statistical interpretation $[19,31]$.

The two alternative strategies of top-down and bottom-up strongly influence the direction of various explorations in the field of quantum mechanism. The Lagrange expression emphasizes single particles in a bottom-up strategy. In contrast, the Euler expression emphasizes complex objects treated as ensembles in a top-down strategy.

From as early as the turn of the 20th century when Plank started his quantum revolution, various interpretations of quantum behaviors have been explored. Following the Heisenberg matrix approach and the Schrödinger wave function equation, the intellectually absorbing anomalies of quantum mechanics have been linked to intrinsic behaviors associated with particle and wave duality.

To address the various paradoxes encountered in the development of quantum mechanics during the course of 20th century, a number of different interpretations may be listed $[19,31]$.

Probabilistic interpretation Max Born 1926 [19]]

Copenhagen interpretation N. Bohr and Heisenberg 1927 [8]

Double-solution interpretation de Broglie 1927, 1953 [9] 
de Broglie-Bohm Theory de Broglie 1927, David Bohm 1952 [9]

Standard interpretation von Neumann 1932 Wigner, Wheeler [32]

Quantum Logic G. Birkhoff and von Neumann 1936 [19]

Ensemble interpretation D. Blokhintsev 1949 [6]

Many-world interpretation H. Everett 1957 [19]

Time-symmetric theory Y. Aharonov 1964

Stochastic interpretation E.Nelson 1966

Many-minds interpretation H. Zeh 1970 [34]

Consistent histories R. Griffiths 1984

Objective collapse theories Ghirardi-Rimini-Weber 1986

Transitional interpretation J. Cramer 1986

Rational interpretation C. Rovelli 1994

In general, the seven key interpretations (Copenhagen, Double-solution, de Broglie-Bohm, Standard, Ensemble, Many-world, and Stochastic) of the first four decades of the 20th century can be separated as follows into the following two general categories $[9,31]$ :

1) Lagrange Expression: comprising the Copenhagen Interpretation (N. Bohr and Heisenberg), the Double-solution (de Broglie), the Standard Interpretation (von Neumann), the Many-world interpretation (H. Everett), and the de Broglie-Bohm Theory (de Broglie \& David Bohm)

2) Euler Expression: comprising the Double-solution (de Broglie), the Ensemble interpretation (D. Blokhintsev), and the Stochastic interpretation (E. Nelson)

In general, a Lagrange expression is preferred for representing a single quanta while a Euler expression can better describe certain group activities. It is interesting to note that de Broglie's Double-solution with a special interpretation can to be involved in both cases $[9,31]$.

According to Einstein's criteria for quantum mechanics [10], an interpretation of quantum mechanics can be characterized by its treatment of:

- Realism

- Completeness

- Local realism

- Determinism

Here, an interpretation is taken to mean a correspondence between the elements of the mathematical formalism $\mathbf{M}$ and the elements of an interpreting structure $\mathbf{I}$, where:

The Mathematical formalism $\mathbf{M}$ consists of the Hilbert space machinery of ket-vectors, self-adjoint operations on the space of ket-vectors, unitary time dependence of the ket-vectors and measurement operations: and ... 
The interpreting structure I includes states, transitions between states, measurement operations and possible information about spatial extension of these elements.

Applying Einstein's criteria to this set of interpretations, the ensemble interpretation (statistical interpretation) is a minimalist interpretation. It claims to make the fewest assumptions associated with the standard mathematics. The most notable supporter of such statistical interpretation was Einstein himself $[19,22,31]$.

\subsubsection{Statistical Interpretation of Quantum Mechanics}

At the 1927 Solvay Congress, Einstein proposed a statistical interpretation in order to avoid conceptual difficulties if the reduction of a wave packet led to the association of wave functions with individual systems. He hoped that someday a complete theory of microphysics would become available to establish a conceptual base as a (preferred) alternative to modern quantum mechanics $[9,31]$.

In 1932, von Neumann established mathematical foundations for quantum mechanics as a standard interpretation on Hilbert space to provide a proof rejecting any hidden variable approach [32].

Influenced by K.V. Nikolskii and V.A. Fock, D. I. Blokhintsev developed a statistical interpretation in the 1940s. He expressed the view that modern quantum mechanics is not a theory of micro-processes but rather a means of studying their properties by the application of statistical ensembles. Menawhile, the approach taken in the publication was borrowed from classical macro physics [6, 19, 22].

Landé's 1951 book sought to reconcile the contradictions between the two classical concepts of the particle and the wave by providing something equivalent to the descriptions of physical phenomena in either terms. He emphasized that in diffraction experiments, particles exhibit both maximum and minimum intensities of diffraction through a perfectly normal mechanical process that can be described in terms of a wave explanation. Using transition probability, these experimentally-determinable transition probabilities can be shown to map a matrix [19].

\subsubsection{Main weaknesses in key interpretations}

Compared to continuous approaches, Heisenberg's matrix offers several advances in handling the case of a single particle. In July 1926, the first question Heisenberg asked Schrödinger was, "Can you use your continuous wave equation to explain black body radiation or quantum effects in photoelectric actions?"

Due to the inherent differences between the two strategies it is difficult to find a direct answer to the question under the Copenhagen interpretation, "Is the Schrödinger equation a single particle description or an equation for a group of particles?" [16, 19, 31].

Through statistical interpretation is a minimalist interpretation, it too is not a complete interpretation. During the development of statistical interpretation there were various debates between Blokhintsev and Heisenberg during the 1940s [19, 22].

Heisenberg questioned as self contradictory, Blokhintsev's basic contention that quantum mechanics eliminates the observer and becomes objectively significant due to the fact that the 
wave function does not describe the state of a particle but rather identifies that the particle belongs to a particular ensemble. In this, Heisenberg argued that in order to assign a particle to a particular ensemble, some knowledge of the particle is required on the part of the observer [19], p445.

The main weakness of Blokhintsev's ensemble interpretation is that though its mathematical formula can express wave distributions well it fails to describe particle structure properly. This is a common weakness of similar mathematical constructions based on periodic components of a Fourier series [19, 27, 31].

Similar to difficulties faced by the Schrödinger equation, ensemble construction is suitable for wave representations but is weak in particle description. On the other hand, the Copenhagen interpretation is preferred for a single particle but comes with inherent limitations of expression with respect to wave behaviours which require further reliance on Born's probabilistic interpretation of the wave-function.

\subsubsection{Other Challenges on Statistical Mechanics}

Statistical mechanics presents us with several fundemental difficulties [20, 23, 24, 31, 33]:

Ergodic property: a time sequence average over a large set of local measurements be replaced by space (phase)-average

Analytic apparatus the construction of asymptotic formulas.

Computational Efficiency: use of modern computing power in tackling complexity.

Discrete via continuous: relationships between irregular discrete systems and regular continuous systems.

Logic foundation: solid logic foundation for statistical mechanics.

\subsection{Chapter organization}

In this chapter, variant construction comprising variant logic, variant measurement and variant phase space is explored with a view to addressing the main challenges and difficulties associated with statistical interpretations and statistical mechanics. The focus is on a unified model to illustrate a path leading from local measurements to global matrices on phase space via variant construction.

This chapter is organized into 12 sections addressing the following:

1. general introduction (above)

2. system architecture

3. creating micro ensemble

4. canonical ensemble and interactive maps

5. global ensemble and interactive map matrices

6. representation models

7. symbolic representation on selected cases 
8. sample results

9. analysis of visual distributions

10. global symmetry properties

11. main results

12. conclusions

\section{System architecture}

In this section, system architecture and its core components are discussed with the use of diagrams.

\subsection{Architecture}

The three components of a Variant Phase Space System are the Creating Micro Ensemble (CME), the Canonical Ensemble (CEIM) together with the Interactive Map and the Global Ensemble Matrix (GEM) as shown in Figure 1. The architecture is shown in Figure 1(a) with the key modules of the three core components being shown in Figures 1(b) through 1(d) respectively.

In the first part of the system, a micro ensemble and its eight projections are created for a given vector and function by the CME component. Next, in order to exhaust all possible $2^{N}$ vectors, a CE and eight IMs are established by the CEIM component. Then, in order to exhaust all possible $2^{2^{n}}$ functions, a CE matrix and eight IM matrices are generated by the GEM component.

With eight parameters in an input group, there are four parameters in the intermediate group and two parameters in the output group.

The three groups of parameters may be listed as follows.

\section{Input group:}

$N$ an integer indicates a $0-1$ vector with $N$ elements

$n$ an integer indicates $n$ variables for a function

$X$ a $0-1$ vector with $N$ elements, $X \in B_{2}^{N}$

$\forall X$ exhaustive set of all states of $N$ bit vectors with $2^{N}$ elements

$J$ a function with $n$ variables, $J \in B_{2}^{2^{n}}$

$\forall J$ exhaustive set of all functions of $n$ variables with $2^{2^{n}}$ elements

$S M$ a selection on a pair of measurements

FC A given configuration for variant logic functions: a $2^{2^{n-1}} \times 2^{2^{n-1}}$ matrix

\section{Intermediate group:}

$M E(J, X)$ a micro ensemble under either multiple or conditional probability measurements $I P(J, X)$ a set of eight interactive projections related to $M E(J, X)$

$C E(J)$ a canonical ensemble for an $N$ bit vector under an $n$ variable function $J$

$I M(J)$ a set of eight interactive maps associated with one $C E(J)$ 


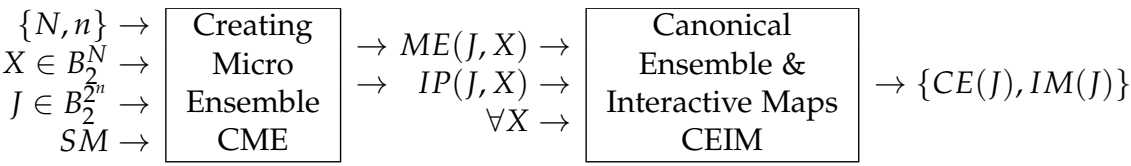

$$
\begin{aligned}
& \begin{aligned}
\{C E(J), I M(J)\} & \rightarrow \text { Global } \\
\forall J & \rightarrow \text { Ensemble } \\
\text { FC } & \rightarrow \text { Matrices } \\
\text { GEM } & \rightarrow \text { CEM }
\end{aligned}
\end{aligned}
$$

(a) Architecture

\begin{tabular}{|c|c|c|c|}
\hline$\{N, n\} \rightarrow$ & Variant & & Probability \\
\hline $\begin{array}{l}X \in B_{2}^{N} \rightarrow \\
J \in B_{2}^{2^{n}} \rightarrow\end{array}$ & $\begin{array}{c}\text { Measures } \\
\text { VM }\end{array}$ & $\rightarrow V M(J, X) \rightarrow$ & $\begin{array}{c}\text { Measurements } \\
\text { PM }\end{array}$ \\
\hline
\end{tabular}

$$
\begin{array}{r}
P M(J, X) \rightarrow \\
S M \rightarrow \begin{array}{c}
\text { Micro } \\
\text { Ensemble } \\
\mathrm{ME}
\end{array} \rightarrow \begin{array}{c}
\text { Interactive } \\
\text { Projection } \\
\mathrm{IP}
\end{array} \rightarrow \operatorname{ME}(J, X) \\
\rightarrow I P(J, X)
\end{array}
$$

(b) CME Creating Micro Ensemble Component

$$
\begin{array}{rl|c|}
M E(J, X) & \rightarrow & \text { Canonical } \\
I P(J, X) & \rightarrow & \text { Ensemble } \\
\forall X & \rightarrow & \text { CE }
\end{array} \rightarrow \begin{gathered}
\text { Interactive } \\
\text { Maps } \\
\text { IM }
\end{gathered} \rightarrow\{C E(J), I M(J)\}
$$

(c) CEIM Canonical Ensemble and Interactive Map Component

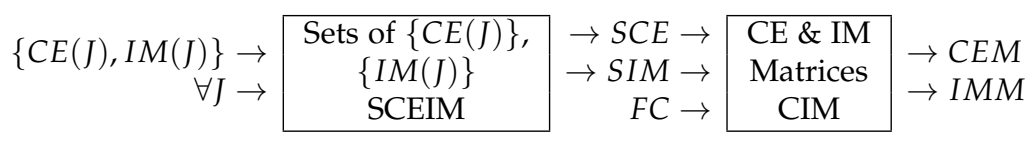

(d) GEM Global Ensemble Matrix Component

Figure 1. (a-d) Variant Phase Space System; (a) Architecture; (b) CME Creating Micro Ensemble; (c) CEIM Canonical Ensemble and Interactive Map; (d) GEM Global Ensemble Matrix

\section{Output group:}

CEM one CE matrix under FC condition

IMM a set of eight IM matrices under FC condition

\subsection{CME creating micro ensemble}

The CME component as shown in Figure 1(b) is composed of four modules: VM Variant Measures, PM Probability Measurements, ME Micro Ensemble and IP Interactive Projection. Five distinct parameters are shown as input signals $\{N, n, X, J, S M\}$ and two groups of vector measurements are performed as a group of output signals $\{M E(J, X), I P(J, X)\}$ respectively. The various parameter can be described as follows: 


\section{Input:}

$N$ an integer indicating a $0-1$ vector with $N$ elements

$n$ an integer indicating $n$ variables for a function

$X$ a $0-1$ vector with $N$ elements, $X \in B_{2}^{N}$

$J$ a function with $n$ variables, $J \in B_{2}^{2^{n}}$

$S M$ a selection on a pair of measurements

\section{Output:}

$M E(J, X)$ a micro ensemble under either multiple or conditional probability measurements $I P(J, X)$ a set of eight interactive projections under the SM condition

A point in variant phase space can be determined under a set of conditions. A set of relevant projections can be associated with an interactive environment. The operation of this module transfers each set of input parameters to one micro ensemble signal and its distinct interactive projections subject to certain restrictions.

\subsection{CEIM canonical ensemble and interactive map}

The CEIM component as shown in Figure 1(c) is composed of two modules: CE Canonical Ensemble and IP Interactive Projection. This component inputs three groups of parameters $\{M E(J, X), I P(J, X), \forall X\}$ from the CME component and outputs two sets of canonical ensembles together with its interactive maps $\{C E(J), I M(J)\}$ as distinct distributions under certain environments. One additional input and two output parameters are described as follows:

\section{Adding Input:}

$\forall X$ exhaustive set of all states of $N$ bit vectors with $2^{N}$ elements

\section{Output:}

$C E(J)$ a canonical ensemble for an $N$ bit vector under an $n$ variable function $J$

$I M(J)$ a set of eight interactive maps associated with $C E(J)$

The CEIM component collects all possible micro ensembles for a given function to form a canonical ensemble on variant phase space. Meanwhile, different interactive maps associated with this CE can be calculated to output as a set of $I M(J)$ as distinct distributions under certain environment.

\subsection{GIM global ensemble and interactive map matrix}

The GIM component as shown in Figure 1(d) is composed of two modules: one for the SCEIM Set of Cannonical Ensembles together with Interactive Maps, and the other for the CIM CE $\mathcal{E}$ IM Matrices.

Two outputs $\{C E(J), I M(J)\}$ from CEIM are taken as inputs, while another two parameters $\{\forall J, F C\}$ and two outputs can be described as follows: 


\section{Adding Input:}

$\forall J$ exhaustive set of all functions of $n$ variables with $2^{2^{n}}$ elements

FC a given configuration for variant logic functions: a $2^{2^{n-1}} \times 2^{2^{n-1}}$ matrix

\section{Output:}

CEM a CE matrix under FC condition

$I M M$ a set of IM matrices under FC condition

The SCEIM module processes an exhaustive operation on all possible values of function $J$ to generate sets of $\{\{C E(J)\},\{I M(J)\}\}_{\forall J}$ as the output. The CIM module further organizes the data to arrange each set as a $2^{2^{n-1}} \times 2^{2^{n-1}}$ matrix with $2^{2^{n}}$ elements and with the specific arrangement determined by $F C$ condition.

After two exhaustive processes through CEIM and GIM activities, a CE matrix and the relevant IM Matrices are generated. Each matrix contains $2^{2^{n}}$ elements as distributions. Further symmetry properties can be identified from each specific configuration.

Since specific components and modules are relevant to the detail of the complex mechanisms, further explanations on each component are presented in Sections 3 through 5.

\section{Creating micro ensemble}

The first part of the system is the CME component composed of four modules: VM Variant Measures, PM Probability Measurements, ME Micro Ensemble and IP Interactive Projection respectively.

It is necessary to clearly describe these four modules in order to understand the measurement properties of variant construction [35]-[44]. Relevant information and supporting materials on fundamental levels of variant construction are briefly descried in Section 3.1 and the four modules are investigated in Sections 3.2 through 3.5.

\subsection{Initial preparation on variant measurements}

The variant measurement construction is based on $n$-variable logic functions and $N$ bit vectors taken as input and output results $[40,43,44]$.

\subsubsection{Two sets of states}

For n-variables where $x=x_{n-1} \ldots x_{i} \ldots x_{0}, 0 \leq i<n, x_{i} \in\{0,1\}=B_{2}$, let a position $j$ be the selected variable $0 \leq j<n, x_{j}$ be the selected variable. Let output variable $y$ and $n$-variable function $f, y=f(x), y \in B_{2}, x \in B_{2}^{n}$. For all states of $x$, a set $S(n)$ composed of the $2^{n}$ states can be divided into two sets: $S_{0}(n)$ and $S_{1}(n)$.

$$
\left\{\begin{array}{c}
S_{0}(n)=\left\{x \mid x_{j}=0, \forall x \in B_{2}^{n}\right\} \\
S_{1}(n)=\left\{x \mid x_{j}=1, \forall x \in B_{2}^{n}\right\} \\
S(n)=\left\{S_{0}(n), S_{1}(n)\right\}
\end{array}\right.
$$




\subsubsection{Four variant functions}

For a given logic function $f$, input and output pair relationships define four variant logic functions $\left\{f_{\perp}, f_{+}, f_{-}, f_{\top}\right\}$.

$$
\left\{\begin{array}{l}
f_{\perp}(x)=\left\{f(x) \mid x \in S_{0}(n), y=0\right\} \\
f_{+}(x)=\left\{f(x) \mid x \in S_{0}(n), y=1\right\} \\
f_{-}(x)=\left\{f(x) \mid x \in S_{1}(n), y=0\right\} \\
f_{\top}(x)=\left\{f(x) \mid x \in S_{1}(n), y=1\right\}
\end{array}\right.
$$

\subsubsection{Two polarized functions}

Considering two standard logic canonical expressions: AND-OR form is selected from $\left\{f_{+}(x), f_{\top}(x)\right\}$ as $y=1$ items, and OR-AND form is selected from $\left\{f_{-}(x), f_{\perp}(x)\right\}$ as $y=0$ items. Considering $\left\{f_{\top}(x), f_{\perp}(x)\right\}, x_{j}=y$ items, they are invariant themselves.

To select $\left\{f_{+}(x), f_{-}(x)\right\}, x_{j} \neq y$ in forming a variant logic expression. Let $f(x)=\left\langle f_{+}|x| f_{-}\right\rangle$ be the variant logic expression. Any logic function can be expressed as a variant logic form. In $\left\langle f_{+}|x| f_{-}\right\rangle$structure, $f_{+}$selected 1 items in $S_{0}(n)$ as same as the AND-OR standard expression, and $f_{-}$selected 0 items in $S_{1}(n)$ as same as OR-AND expression.

\subsection{4. $n=2$ representation}

For a convenient understanding of the variant representation, 2-variable logic structures are illustrated in Table 2 for its 16 functions in four variant functions as follows.

Let $x^{v}=\left\{\begin{array}{l}\perp, x=0, y=0 ; \\ +, x=0, y=1 ; \\ -, x=1, y=0 ; \\ \top, x=1, y=1\end{array}\right.$ and $x^{\delta}=\left\{\begin{array}{l}x, \delta=1 ; \\ \bar{x}, \delta=0 .\end{array}\right.$

For a pair of $\left\{f_{+}, f_{-}\right\}$functions selected from the structure, relevant representations are illustrated in Table 3 to show the variant capacity on the full expression of all logic functions.

Checking two functions $f=3$ and $f=6$ respectively.

$\left\{f=3:=\{1,0\}, f_{+}=11:=\langle 0 \mid \varnothing\rangle, f_{-}=2:=\langle\varnothing \mid 3\rangle\right\}$;

$\left\{f=6:=\{2,1\}, f_{+}=14:=\langle 2 \mid \varnothing\rangle, f_{-}=2:=\langle\varnothing \mid 3\rangle\right\}$.

\subsubsection{Variant measure functions}

Let $\Delta$ be the variant measure function [1,35]-[42]

$$
\begin{aligned}
\Delta & =\left\langle\Delta_{\perp}, \Delta_{+}, \Delta_{-}, \Delta_{\top}\right\rangle \\
\Delta J(x) & =\left\langle\Delta_{\perp} J(x), \Delta_{+} J(x), \Delta_{-} J(x), \Delta_{\top} J(x)\right\rangle \\
\Delta_{\alpha} J(x) & =\left\{\begin{array}{l}
\left.1, J(x) \in J_{\alpha}(x), \alpha \in\{\perp,+,-,\}\right\} \\
0, \text { others }
\end{array}\right.
\end{aligned}
$$

For any given $n$-variable state there is one position in $\Delta J(x)$ to be 1 and other 3 positions are 0 . 


\begin{tabular}{c|c|cccc|cccc|cccc}
\hline$f$ & $f \in$ & 3 & 2 & 1 & 0 & $3^{v}$ & $2^{v}$ & $1^{v}$ & $0^{v}$ & $f_{\perp} \in$ & $f_{+} \in$ & $f_{-} \in$ & $f_{\top} \in$ \\
No. & $S(n)$ & 11 & 10 & 01 & 00 & $11^{v}$ & $10^{v}$ & $01^{v}$ & $00^{v}$ & $S_{0}(n)$ & $S_{0}(n)$ & $S_{1}(n)$ & $S_{1}(n)$ \\
\hline 0 & $\{\varnothing\}$ & 0 & 0 & 0 & 0 & - & $\perp$ & - & $\perp$ & $\{2,0\}$ & $\{\varnothing\}$ & $\{3,1\}$ & $\{\varnothing\}$ \\
1 & $\{0\}$ & 0 & 0 & 0 & 1 & - & $\perp$ & - & + & $\{2\}$ & $\{0\}$ & $\{3,1\}$ & $\{\varnothing\}$ \\
2 & $\{1\}$ & 0 & 0 & 1 & 0 & - & $\perp$ & $\top$ & $\perp$ & $\{2.0\}$ & $\{\varnothing\}$ & $\{3\}$ & $\{1\}$ \\
3 & $\{1,0\}$ & 0 & 0 & 1 & 1 & - & $\perp$ & $\top$ & + & $\{2\}$ & $\{0\}$ & $\{3\}$ & $\{1\}$ \\
4 & $\{2\}$ & 0 & 1 & 0 & 0 & - & + & - & $\perp$ & $\{0\}$ & $\{2\}$ & $\{3,1\}$ & $\{\varnothing\}$ \\
5 & $\{2,0\}$ & 0 & 1 & 0 & 1 & - & + & - & + & $\{\varnothing\}$ & $\{2,0\}$ & $\{3,1\}$ & $\{\varnothing\}$ \\
6 & $\{2,1\}$ & 0 & 1 & 1 & 0 & - & + & $\top$ & $\perp$ & $\{0\}$ & $\{2\}$ & $\{3\}$ & $\{1\}$ \\
7 & $\{2,1,0\}$ & 0 & 1 & 1 & 1 & - & + & $\top$ & + & $\{\varnothing\}$ & $\{2,0\}$ & $\{3\}$ & $\{1\}$ \\
8 & $\{3\}$ & 1 & 0 & 0 & 0 & $\top$ & $\perp$ & - & $\perp$ & $\{2,0\}$ & $\{\varnothing\}$ & $\{1\}$ & $\{3\}$ \\
9 & $\{3,0\}$ & 1 & 0 & 0 & 1 & $\top$ & $\perp$ & - & + & $\{2\}$ & $\{0\}$ & $\{1\}$ & $\{3\}$ \\
10 & $\{3,1\}$ & 1 & 0 & 1 & 0 & $\top$ & $\perp$ & $\top$ & $\perp$ & $\{2,0\}$ & $\{\varnothing\}$ & $\{\varnothing\}$ & $\{3,1\}$ \\
11 & $\{3,1,0\}$ & 1 & 0 & 1 & 1 & $\top$ & $\perp$ & $\top$ & + & $\{2\}$ & $\{0\}$ & $\{\varnothing\}$ & $\{3,1\}$ \\
12 & $\{3,2\}$ & 1 & 1 & 0 & 0 & $\top$ & + & - & $\perp$ & $\{0\}$ & $\{2\}$ & $\{1\}$ & $\{3\}$ \\
13 & $\{3,2,0\}$ & 1 & 1 & 0 & 1 & $\top$ & + & - & + & $\{\varnothing\}$ & $\{2,0\}$ & $\{1\}$ & $\{3\}$ \\
14 & $\{3,2,1\}$ & 1 & 1 & 1 & 0 & $\top$ & + & $\top$ & $\perp$ & $\{0\}$ & $\{2\}$ & $\{\varnothing\}$ & $\{3,1\}$ \\
15 & $\{3,2,1,0\}$ & 1 & 1 & 1 & 1 & $\top$ & + & $\top$ & + & $\{\varnothing\}$ & $\{2,0\}$ & $\{\varnothing\}$ & $\{3,1\}$ \\
\hline
\end{tabular}

Table 2. Four Variant Functions in 2-variable logic

\begin{tabular}{c|c|cccc|r|rrrr|l}
\hline$f$ & $f \in$ & 3 & 2 & 1 & 0 & $f_{+} \in$ & $3^{0}$ & $2^{1}$ & $1^{0}$ & $0^{1}$ & $f-\epsilon$ \\
No. & $S(n)$ & 11 & 10 & 01 & 0 & $S_{0}(n)$ & $11^{0}$ & $10^{1}$ & $01^{0}$ & $00^{1}$ & $S_{1}(n)$ \\
\hline 0 & $\{\varnothing\}$ & 0 & 0 & 0 & 0 & $\langle\varnothing|$ & 1 & 0 & 1 & 0 & $|3,1\rangle$ \\
1 & $\{0\}$ & 0 & 0 & 0 & 1 & $\langle 0|$ & 1 & 0 & 1 & 1 & $|3,1\rangle$ \\
2 & $\{1\}$ & 0 & 0 & 1 & 0 & $\langle\varnothing|$ & 1 & 0 & 0 & 0 & $|3\rangle$ \\
3 & $\{1,0\}$ & 0 & 0 & 1 & 1 & $\langle 0|$ & 1 & 0 & 0 & 1 & $|3\rangle$ \\
4 & $\{2\}$ & 0 & 1 & 0 & 0 & $\langle 2|$ & 1 & 1 & 1 & 0 & $|3,1\rangle$ \\
5 & $\{2,0\}$ & 0 & 1 & 0 & 1 & $\langle 2,0|$ & 1 & 1 & 1 & 1 & $|3,1\rangle$ \\
6 & $\{2,1\}$ & 0 & 1 & 1 & 0 & $\langle 2|$ & 1 & 1 & 0 & 0 & $|3\rangle$ \\
7 & $\{2,1,0\}$ & 0 & 1 & 1 & 1 & $\langle 2,0|$ & 1 & 1 & 0 & 1 & $|3\rangle$ \\
8 & $\{3\}$ & 1 & 0 & 0 & 0 & $\langle\varnothing|$ & 0 & 0 & 1 & 0 & $|1\rangle$ \\
9 & $\{3,0\}$ & 1 & 0 & 0 & 1 & $\langle 0|$ & 0 & 0 & 1 & 1 & $|1\rangle$ \\
10 & $\{3,1\}$ & 1 & 0 & 1 & 0 & $\langle\varnothing|$ & 0 & 0 & 0 & 0 & $|\varnothing\rangle$ \\
11 & $\{3,1,0\}$ & 1 & 0 & 1 & 1 & $\langle 0|$ & 0 & 0 & 0 & 1 & $|\varnothing\rangle$ \\
12 & $\{3,2\}$ & 1 & 1 & 0 & 0 & $\langle 2|$ & 0 & 1 & 1 & 0 & $|1\rangle$ \\
13 & $\{3,2,0\}$ & 1 & 1 & 0 & 1 & $\langle 2,0|$ & 0 & 1 & 1 & 1 & $|1\rangle$ \\
14 & $\{3,2,1\}$ & 1 & 1 & 1 & 0 & $\langle 2|$ & 0 & 1 & 0 & 0 & $|\varnothing\rangle$ \\
15 & $\{3,2,1,0\}$ & 1 & 1 & 1 & 1 & $\langle 2,0|$ & 0 & 1 & 0 & 1 & $|\varnothing\rangle$ \\
\hline \multicolumn{10}{l}{}
\end{tabular}

Table 3. A pair of selected functions and their full expression 


\subsubsection{Variant measures on vector}

For any $N$ bit $0-1$ vector $X, X=X_{N-1} \ldots X_{j} \ldots X_{0}, 0 \leq j<N, X_{j} \in B_{2}, X \in B_{2}^{N}$ under $n$-variable function $J, n$ bit 0-1 output vector $Y, Y=J(X)=\left\langle J_{+}|X| J_{-}\right\rangle, Y=Y_{N-1} \ldots Y_{j} \ldots Y_{0}, 0 \leq j<$ $N, Y_{j} \in B_{2}, Y \in B_{2}^{N}$. For the $j$-th position $x^{j}=\left[\ldots X_{j} \ldots\right] \in B_{2}^{n}$ to form $Y_{j}=J\left(x^{j}\right)=\left\langle J_{+}\left|x^{j}\right| J_{-}\right\rangle$. Let $N$ bit positions be cyclic linked. Variant measures of $J(X)$ can be decomposed as a quaternion

$$
\Delta(X: Y)=\Delta J(X)=\sum_{j=0}^{N-1} \Delta J\left(x^{j}\right)=\left\langle N_{\perp}, N_{+}, N_{-}, N_{\top}\right\rangle
$$

$\left\langle N_{\perp}, N_{+}, N_{-}, N_{\top}\right\rangle, N=N_{\perp}+N_{+}+N_{-}+N_{\top}$.

\subsubsection{Example}

E.g. $N=12$, given $J, Y=J(X)$.

$$
\begin{aligned}
& X=1 \quad 0 \begin{array}{llllllllll}
X & 1 & 1 & 0 & 1 & 1 & 1 & 0 & 0 & 1
\end{array} \\
& Y=0 \begin{array}{llllllllllll}
0 & 0 & 1 & 0 & 1 & 0 & 1 & 0 & 1 & 1 & 0 & 0
\end{array} \\
& \Delta(X: Y)=-\perp \top-\top \perp \top-\top+\perp-
\end{aligned}
$$

$\Delta J(X)=\left\langle N_{\perp}, N_{+}, N_{-}, N_{\top}\right\rangle=\langle 3,1,4,4\rangle, N=12$

Input and output pairs are 0-1 variables for only four combinations. For any given function $J$, the quantitative relationship of $\{\perp,+,-, \top\}$ is directly derived from the input/output sequences. Four meta measures are determined.

\subsubsection{Basic Properties of Variant Logic}

For given $n$ 0-1 variables, a given function $J$ and an $N$ bit vector $X$, the following corollaries can be described [35]-[44].

Corollary 3.1: For $n$ 0-1 variables, the state set contains a total of $2^{n}$ states.

Corollary 3.2: For $n$ 0-1 variables, the function set contains a total of $2^{2^{n}}$ functions.

Corollary 3.3: For an $N$ bit vector $X$, the phase space is composed of a total of $2^{N}$ vectors.

Corollary 3.4: A logic function $f$ can be partitioned as four variant functions as $f=$ $\left(f_{\perp}, f_{+}, f_{-}, f_{\top}\right)$ respectively.

Corollary 3.5: For a given vector $X$ and a given function $J$, a measure vector of four meta measures for variant measures can be determined as a quaternion $\Delta J(X)=$ $\left\langle N_{\perp}, N_{+}, N_{-}, N_{\top}\right\rangle$.

\subsection{VM variant measures}

Using defined variant functions, it is possible to describe the VM module in Fig. 1(b) as follows. 
Under variant construction, $N$ bits of $0-1$ vector $X$ under a function $J$ produce seven Meta measures composed of a measure vector $V M(J, X)$.

$$
\begin{aligned}
(X: J(X)) & \rightarrow\left(N_{\perp}, N_{+}, N_{-}, N_{\top}\right), \\
N_{0} & =N_{\perp}+N_{+}, \\
N_{1} & =N_{-}+N_{\top}, \\
N & =N_{0}+N_{1} .
\end{aligned}
$$

From a measuring viewpoint, there are seven measures identified in this set of parameters. They can be expressed in three levels.

$$
N_{\perp}{ }^{N_{0}} N_{+} \quad N_{-}{ }^{N_{1}} N_{\top}
$$

In the current system, the output of the VM module is expressed as $\operatorname{VM}(J, X)=$ $\left\{N_{\perp}, N_{+}, N_{-}, N_{\top}, N_{0}, N_{1}, N\right\}$.

\subsection{PM probability measurements}

Measures of $\operatorname{VM}(J, X)$ are input as numeric vectors into the PM module. Using variant quaternion and other three core measures, local measurements of probability signals are calculated as eight meta measurements in two groups by following the given equations. For any $N$ bit 0-1 vector $X$, function $J$, under $\Delta$ measurement: $\Delta J(X)=\left\langle N_{\perp}, N_{+}, N_{-}, N_{\top}\right\rangle$, $N_{0}=N_{\perp}+N_{+}, N_{1}=N_{-}+N_{\top}, N=N_{0}+N_{1}$

The first group of probability signal vectors $\rho$ and $\left\{\rho_{0}, \rho_{1}\right\}$ are defined by

$$
\left\{\begin{array}{l}
\rho=\frac{\Delta J(X)}{N}=\left(\rho_{\perp}, \rho_{+}, \rho_{-}, \rho_{\top},\right) \\
\rho_{\alpha}=\frac{N_{\alpha}}{N}, \alpha \in\{\perp,+,-, \top\} \\
\rho_{0}=N_{0} / N \\
\rho_{1}=N_{1} / N .
\end{array}\right.
$$

The second group of probability signal vectors $\tilde{\rho}$ and $\left\{\tilde{\rho}_{0}, \tilde{\rho}_{1}\right\}$ is defined by

$$
\left\{\begin{aligned}
\tilde{\rho} & =\frac{\Delta J(X)}{N_{0} \mid N_{1}}=\left(\tilde{\rho}_{\perp}, \tilde{\rho}_{+}, \tilde{\rho}_{-}, \tilde{\rho}_{\top}\right), \\
\tilde{\rho}_{\perp} & =\frac{N_{\perp}}{N_{0}} \\
\tilde{\rho}_{+} & =\frac{N_{+}}{N_{0}} \\
\tilde{\rho}_{-} & =\frac{N_{-}}{N_{1}} \\
\tilde{\rho}_{\top} & =\frac{N_{T}}{N_{1}} \\
\tilde{\rho}_{0} & =N_{0} / N, \\
\tilde{\rho}_{1} & =N_{1} / N .
\end{aligned}\right.
$$

The two groups of probability measurements are key components in variant measurement. The first group corresponds to multiple probability measurements and the second group 
corresponds to conditional probability measurements. In this Chapter, only two quaternion measurements are used in order to focus attention on the simplest interactive combinations without further measurements of $\left\{\rho_{0}, \rho_{1}\right\}$ and $\left\{\tilde{\rho}_{0}, \tilde{\rho}_{1}\right\}$ involved.

Under such condition, the output signals of the PM module can be expressed as a pair of probability vectors in quaternion forms $P M(J, X)=\{\rho, \tilde{\rho}\}$.

\subsection{ME micro ensemble}

The ME module has two inputs. $P M(J, X)$ provides probability measurement vectors to provide the basis of the measurement. The input parameter $S M$ indicates Selected Measurements from $P M(J, X)$.

In this paper, two cases for a pair of measurement selections are restricted to permit an investigation of possible configurations of interactive distributions in their variant phase spaces under simple conditions.

Case A: $\left(p_{i}, p_{j}\right)$ or $\left(p_{+}, p_{-}\right) \in P \subset \rho$ with two measurements from $\rho$;

Case B: $\left(\tilde{p}_{i}, \tilde{p}_{j}\right)$ or $\left(\tilde{p}_{+}, \tilde{p}_{-}\right) \in \tilde{P} \subset \tilde{\rho}$ with two measurements from $\tilde{\rho}$.

Under these conditions, each $\left(p_{i}(J, X), p_{j}(J, X)\right)$ or $\left(\tilde{p}_{i}(J, X), \tilde{p}_{j}(J, X)\right)$ determines a fixed position on variant phase space as a Micro Ensemble. The output of the ME module can be expressed as $M E(J, X)=\left(p_{i}(J, X), p_{j}(J, X)\right) \mid\left(\tilde{p}_{i}(J, X), \tilde{p}_{j}(J, X)\right)$ under a given function $J$, an $N$ bit vector $X$ and $S M$ conditions.

\subsubsection{Variant Phase Space}

Since each ME must be located on a certain position in a square area on variant phase space, it is convenient to show the restrictions and specific properties according to the following propositions.

Proposition 3.1: In the Case A condition, a total of six configurations can be identified in different $P$ selections. For each configuration, its pair of probability measurements can be restricted in a triangle area of a $[0,1]^{2}$ region.

Proof: Any selection of two elements $\left(p_{i}(J, X), p_{j}(J, X)\right)$ from $P$, it satisfies $0 \leq p_{i}(J, X)+$ $p_{j}(J, X) \leq 1$, there are six distinct selections.

Proposition 3.2: In the Case B condition, a total of six configurations can be identified into two groups in different $\tilde{P}$ selections, four configurations in the first group are restricted in a square area and two configurations of the second group are restricted on a diagonal line.

Proof: Since two equations in $\tilde{\rho}$ quaternion are in the conditions of $\tilde{\rho_{\perp}}+\tilde{\rho_{+}}=\tilde{\rho_{-}}+\tilde{\rho_{\bar{T}}}=1$. For the first group, two selected components can satisfy $0 \leq \tilde{p}_{i}, \tilde{p}_{j} \leq 1$, four distinct selections are identified in a $[0,1]^{2}$ restricted region. For the second group, two selected components can satisfy $0 \leq \tilde{p}_{i}, \tilde{p}_{j} \leq 1$ and $\tilde{p}_{i}+\tilde{p}_{j}=1$, only two distinct selections are identified on a diagonal line distributed in a $[0,1]^{2}$ region.

Under this arrangement, all measurements are relevant to variant construction. Now, let this type of phase spaces be Variant Phase Space VPS. For an $N$ bit vector $X$, a pair of probability measurements determines a micro ensemble to be a specific position in VPS. 
In order to distinguish between the two types of VPS, let us name a subset of VPS under Case A conditions as a Multiple Phase Space MPS while we name a subset of VPS under Case B conditions as a Conditional Phase Space CPS. Samples of canonical ensembles of the six combinations under a given SM condition under a function in both MPS \& CPS are shown in Figure 2(I,II) respectively.

\subsection{IP interactive projections}

Using a micro ensemble $M E(J, X)$, different projections can be identified in an IP module under various interactive conditions. Based on the input micro ensemble for each Case, two groups of eight interactive projections can be distinguished by symmetry/anti-symmetry and synchronous/asynchronous conditions.

\subsubsection{Synchronous and Asynchronous Operations}

Each $M E(J, X)$ is a pair of probability measurements and it is essential to establish corresponding rules to place their interactive projection in the same probability region i.e. $[0,1]$ segment.

We can distinguish between Synchronous and Asynchronous time-related operations.

Under a synchronous operation $\{+,-, \times, /$,$\} , only one merged measurement is located in$ $[0,1]$ region to express one activity from a ME.

However, under an asynchronous operation $\oplus$, two input measurements $p_{+} \neq p_{-}$, generate an output result as a vector that has two positions of $p_{+}$and $p_{-}$with a weighted value 1 on each position; when $p=p_{+}=p_{-}$there is a weighted value of 2 on the position $p$.

Under asynchronous operations, merged results may be distinguished by their position or overlap each other with a cumulative weight value of 2 . However, under synchronous operations, two measurements are merged as a unit weight to shift interactive measurements to one position in the $[0,1]$ region.

From an integrative viewpoint, the two types of operations may be considered capable of either merging two particles (asynchronous) on two positions or integrating two waves (synchronous) on a position.

\subsubsection{Case A: Multiple Probability Interactive Projections}

For each $M E(J, P)=\left(p_{i}(J, X), p_{j}(J, X)\right)$ has a position on a unit square $[0,1]^{2}$.

Let $P=\left\{p_{+}, p_{-}\right\}$(or $\left\{p_{x}, p_{y}\right\}$ ) locate a pair of measurements, the IP module projects two measurements and its weight into four conditions in different symmetric properties to form two groups of eight weight vectors as interactive projections.

Using $P=\left\{p_{+}, p_{-}\right\}$, a pair of measurement vectors $\{u, v\}$ are formulated: 
(a)

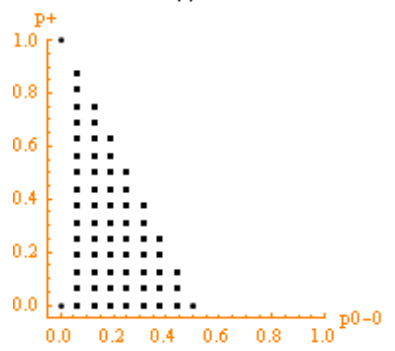

(d)

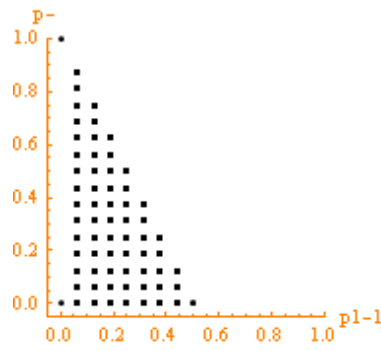

(a)

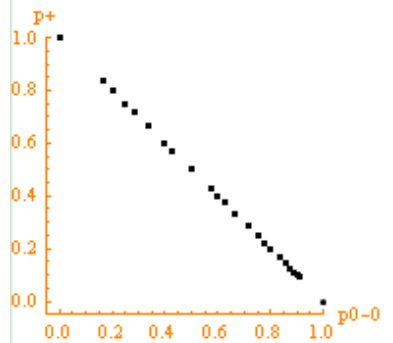

(d)

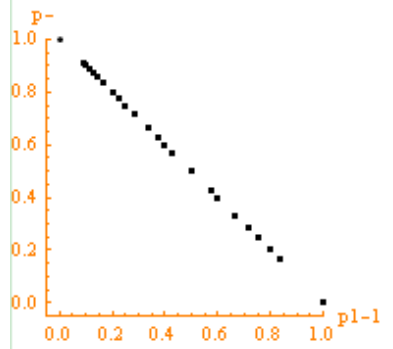

(b)

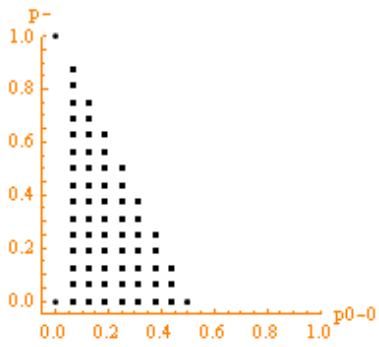

(e)

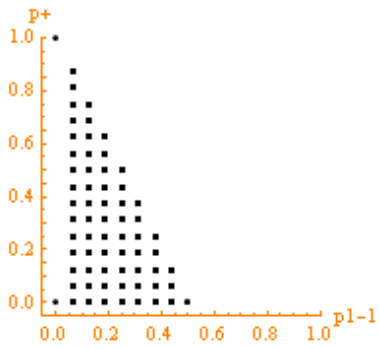

(I) Type A

(b)

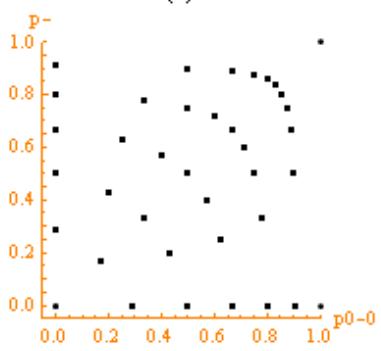

(e)

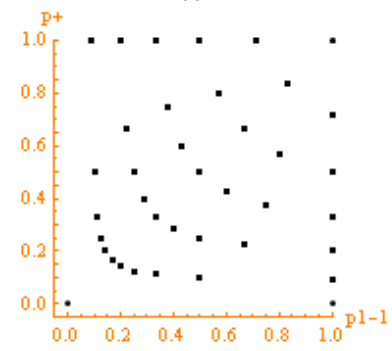

(II) Type B (c)

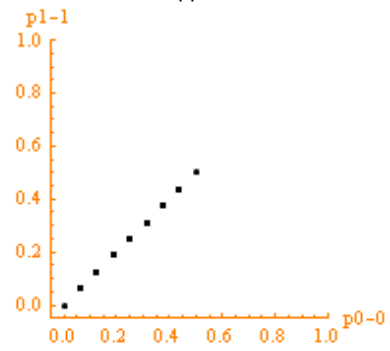

(f)

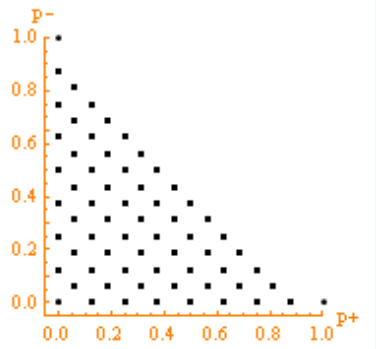

(c)

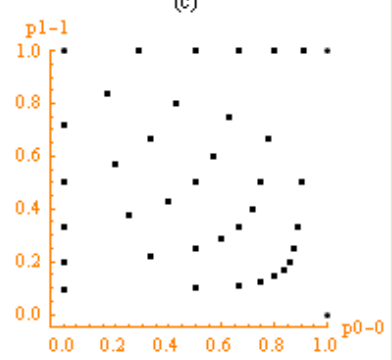

(f)

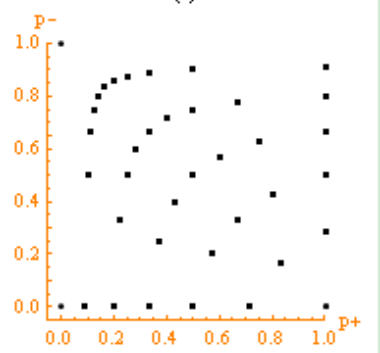
Six combinations in MPS; (II) Type B:(a-f) Six combinations in CPS 


$$
\begin{gathered}
\left\{\begin{array}{l}
u=\left(u_{+}, u_{-}, u_{0}, u_{1}\right)=\left\{u_{\beta}\right\} \\
v=\left(v_{+}, v_{-}, v_{0}, v_{1}\right)=\left\{v_{\beta}\right\} \\
\beta \in\{+,-, 0,1\}
\end{array}\right. \\
\left\{\begin{array}{l}
u_{+}=p_{+} \\
u_{-}=p_{-} \\
u_{0}=u_{+} \oplus u_{-} \\
u_{1}=u_{+}+u_{-} \\
v_{+}=\frac{1+p_{+}}{2} \\
v_{-}=\frac{1-p_{-}}{2} \\
v_{0}=v_{+} \oplus v_{-} \\
v_{1}=v_{+}+v_{-}-0.5
\end{array}\right.
\end{gathered}
$$

where $0 \leq u_{\beta}, v_{\beta} \leq 1, \beta \in\{+,-, 0,1\}, \oplus$ : Asynchronous addition, + : Synchronous addition.

\subsubsection{Case B: Conditional Probability Interactive Projections}

For each $\operatorname{ME}(J, \tilde{P})=\left(\tilde{p}_{i}(J, X), \tilde{p}_{j}(J, X)\right)$ we can note that it has a position on a unit square $[0,1]^{2}$.

Let $\tilde{P}=\left\{\tilde{p}_{+}, \tilde{p}_{-}\right\}$locate a pair of measurements, the IP module projects two measurements and its weight into four conditions in different symmetric properties to form two groups of eight weights as interactive projections.

Using $\tilde{P}=\left\{\tilde{p}_{+}, \tilde{p}_{-}\right\}$, a pair of vectors $\{\tilde{u}, \tilde{v}\}$ are formulated:

$$
\left\{\begin{array}{c}
\tilde{u}=\left(\tilde{u}_{+}, \tilde{u}_{-}, \tilde{u}_{0}, \tilde{u}_{1}\right)=\left\{\tilde{u}_{\beta}\right\} \\
\tilde{v}=\left(\tilde{v}_{+}, \tilde{v}_{-}, \tilde{v}_{0}, \tilde{v}_{1}\right)=\left\{\tilde{v}_{\beta}\right\} \\
\beta \in\{+,-, 0,1\}
\end{array}\right.
$$

For the four projections in a square area, the following equations are required.

$$
\left\{\begin{array}{l}
\tilde{u}_{+}=\tilde{p}_{+} \\
\tilde{u}_{-}=\tilde{p}_{-} \\
\tilde{u}_{0}=\tilde{u}_{+} \oplus \tilde{u}_{-} \\
\tilde{u}_{1}=\frac{\tilde{u}_{+}+\tilde{u}_{-}}{1+\tilde{p}_{+}} \\
\tilde{v}_{+}=\frac{1+\tilde{p}_{+}}{2} \\
\tilde{v}_{-}=\frac{1-\tilde{p}_{-}}{2} \\
\tilde{v}_{0}=\tilde{v}_{+} \oplus \tilde{v}_{-} \\
\tilde{v}_{1}=\tilde{v}_{+}+\tilde{v}_{-}-0.5
\end{array}\right.
$$


For the two projections in a diagonal line, the following equations are satisfied.

$$
\left\{\begin{aligned}
\tilde{u}_{+} & =\tilde{p}_{i} \\
\tilde{u}_{-} & =\tilde{p}_{j} \\
\tilde{u}_{0} & =\tilde{u}_{+} \oplus \tilde{u}_{-} \\
\tilde{u}_{1} & =\tilde{u}_{+}+\tilde{u}_{-} \\
\tilde{v}_{+} & =\frac{1+\tilde{p}_{i}}{2} \\
\tilde{v}_{-} & =\frac{1-\tilde{p}_{j}}{2} \\
\tilde{v}_{0} & =\tilde{v}_{+} \oplus \tilde{v}_{-} \\
\tilde{v}_{1} & =\tilde{v}_{+}+\tilde{v}_{-}-0.5
\end{aligned}\right.
$$

where $0 \leq \tilde{u}_{\beta}, \tilde{v}_{\beta} \leq 1, \beta \in\{+,-, 0,1\}, \oplus$ : Asynchronous addition, + : Synchronous addition.

\subsubsection{Key Properties in IP Module}

Under Symmetry/Anti-symmetry and Synchronous/Asynchronous conditions, one ME corresponds to eight interactive projections to express their selected characteristics.

Proposition 3.3: Two types of distinguished projections can be identified under symmetry/anti-symmetry conditions for each ME.

Proof: For two projection vectors $\{u, v\}$, we can note that $u$ represents a symmetry condition and $v$ represents an anti-symmetry condition.

Proposition 3.4: Synchronous and Asynchronous conditions lead to significant different output results.

Proof: In a synchronous operation, only one unit weight is output as $\left\{u_{1}, v_{1}\right\}$. However, in an asynchronous operation, two positions may be seen to have a combined weight $\left\{u_{0}, v_{0}\right\}$.

Proposition 3.5: Other projections are simple ones corresponding to relevant measurement projections.

Proof: Other output results are $\left\{u_{+}, u_{-}, v_{+}, v_{-}\right\}$, each parameter is only dependent on one measurement to get a similar distribution from a projection viewpoint. There is no real interactive activity involved.

Proposition 3.6: If two probability measurements are required to satisfy $p_{i}+p_{j} \leq 1$, then their symmetry interactive projection result is equal to $p_{i}+p_{j}$.

Proof: Merged result is in the $[0,1]$ region.

Proposition 3.7: If two probability measurements independently have $0 \leq p_{i}, p_{j} \leq 1$, then their symmetry interactive projection is $\left(p_{i}+p_{j}\right) / 2$.

Proof: Under this condition, merged results are in the $[0,1]$ region.

Proposition 3.8 From a selected ME, eight interactive projections can be formulated.

Proof: By Propositions 3.3-3.7. 
Under different conditions, one pair of probability measurements can be interactively projected into eight distinct results. However, from a variant viewpoint, it is not sufficient for a serious analysis to use only a single set of measurements from a ME, further extensions are required.

To distinguish among different measurements in interactive projections, four types of measurements are defined as Left, Right, D-P and $D-W$, where $\left\{u_{+}, v_{+}, \tilde{u}_{+}, \tilde{v}_{+}\right\}$are Left measurements, $\left\{u_{-}, v_{-}, \tilde{u}_{-}, \tilde{v}_{-}\right\}$are Right measurements, $\left\{u_{0}, v_{0}, \tilde{u}_{0}, \tilde{v}_{0}\right\}$ are $D-P$ measurements and $\left\{u_{1}, v_{1}, \tilde{u}_{1}, \tilde{v}_{1}\right\}$ are $D-W$ measurements respectively.

\section{CEIM canonical ensemble and interactive maps}

It is a basic step to generate a micro ensemble and eight interactive projections on variant phase space. For a given function $J$, it is necessary to determine the specific positions of all possible vectors of $\forall X$ to form a canonical ensemble on variant phase space.

The CEIM component is composed of two modules: the CE Canonical Ensemble and the IM Interactive Map.

The CE module collects all possible MEs into a canonical ensemble. In addition, the IM module makes relevant interactive projections via IP's outputs to generate a list of interactive distributions in the relevant maps as output results.

\subsection{CE canonical ensemble}

In the CE module, all the MEs are collected to form a CE in variant phase space according to the following equations.

For a function $J$ and all $2^{N}$ vectors of $\forall X$, let $C E L(J, X)$ be a point of $C E L(J)$ on a plane lattice

$$
\begin{gathered}
C E L(J, X)=\left\{\begin{array}{l}
T, M E(J, X)=P \mid \tilde{P} \\
F, \text { Otherwise }
\end{array}\right. \\
C E L(J)=\bigcup_{\forall X} C E L(J, X)
\end{gathered}
$$

Applying the equation for $C E L(J)$, a canonical lattice $C E L$ can be established to indicate a specific distribution from a logic viewpoint.

Since different $C E L(J, X)$ may have the same position, let $C E(J, X)$ be a point of $C E(J)$ in a canonical ensemble

$$
\begin{gathered}
C E(J, X)=\left\{\begin{array}{l}
1, C E L(J, X)=T \\
0, \text { Otherwise }
\end{array}\right. \\
C E(J)=\sum_{\forall X} C E(J, X)
\end{gathered}
$$


Using equation $C E(J)$, a canonical ensemble of variant phase space is produced. Each non-zero position has a numeric weight as a value to indicate numbers of MEs collected in a position.

\subsubsection{Key Properties in CE}

Proposition 4.1: Under Case A conditions, $O\left(N^{2} / 2\right)$ points may be identified on a $C E L(J)$ lattice.

Proof: For each probability measurement, $N+1$ values may be distinguished; points are located in a triangular area and a total of $(N+1) N / 2$ points may be distinguished.

Proposition 4.2: Under Case B conditions, $O(N)-O\left(N^{2}\right)$ points may be distinguished on a $C E L(J)$ lattice.

Proof: For each probability measurement, $N+1$ values may be distinguished; points are located in a square area and $(N+1)^{2}$ points may be distinguished for four square distributions and $N+1$ points may be distinguished for two diagonal line distributions.

Proposition 4.3: In Case A or Case B, values of all possible points of $C E(J)$ collected are equal to $2^{N}$.

Proof: This is the total number of vectors that may be distinguished for $\forall X$.

Proposition 4.4: For a given $S M$ condition, $C E(J)$ is a statistical canonical ensemble on variant phase space.

Proof: For a given SM condition, a $C E(J)$ distribution is independent of special sequences of collection. Its detailed configuration is relevant to $\{n, N\}$ and $S M$ respectively. All valid positions can be statistically generated.

Under this organization, each $C E(J)$ has a fixed plane lattice with a distinct distribution. This invariant property is useful for our further explorations.

\subsection{IM interactive map}

In an IM module, all possible IP projections of either $\{u, v\}$ or $\{\tilde{u}, \tilde{v}\}$ are collected. Each projection corresponds to a specific IM distribution.

The IM module provides a statistical means to accumulate all possible vectors of $N$ bits for a selected signal and generate a histogram. Eight signals correspond to eight histograms respectively. Among these, four histograms exhibit properties of symmetry and another four histograms exhibit properties of anti-symmetry.

\subsubsection{Statistical distributions}

For a function $J$, all measurement signals are collected from the IP and the relevant histogram represents a complete statistical distribution as an IP map.

Using $u$ and $v$ signals, each $u_{\beta}$ or $v_{\beta}$ determines a fixed position in the relevant histogram to make vector $X$ on a position. After completing $2^{N}$ data sequences, eight 
symmetry/anti-symmetry histograms of $\left\{H\left(u_{\beta} \mid J\right)\right\},\left\{H\left(v_{\beta} \mid J\right)\right\} \mid\left\{H\left(\tilde{u}_{\beta} \mid J\right)\right\},\left\{H\left(\tilde{v}_{\beta} \mid J\right)\right\}$ are generated.

Under the multiple probability condition, $\beta \in\{+,-, 0,1\}$

$$
\left\{\begin{array}{l}
H\left(u_{\beta} \mid J\right)=\sum_{\forall X \in B_{2}^{N}} H\left(u_{\beta} \mid J(X)\right) \\
H\left(v_{\beta} \mid J\right)=\sum_{\forall X \in B_{2}^{N}} H\left(v_{\beta} \mid J(X)\right), J \in B_{2}^{2^{n}}
\end{array}\right.
$$

Under the conditional probability condition, $\beta \in\{+,-, 0,1\}$

$$
\left\{\begin{array}{l}
H\left(\tilde{u}_{\beta} \mid J\right)=\sum_{\forall X \in B_{2}^{N}} H\left(\tilde{u}_{\beta} \mid J(X)\right) \\
H\left(\tilde{v}_{\beta} \mid J\right)=\sum_{\forall X \in B_{2}^{N}} H\left(\tilde{v}_{\beta} \mid J(X)\right), J \in B_{2}^{2^{n}}
\end{array}\right.
$$

\subsubsection{Normalized probability histograms}

Let $|H(.)$.$| denote the total number in the histogram H(.$.$) , a normalized probability$ histogram $\left(P_{H}(.).\right)$ can be expressed as

$$
\left\{\begin{array}{l}
P_{H}\left(u_{\beta} \mid J\right)=\frac{H\left(u_{\beta} \mid J\right)}{\left|H\left(u_{\beta} \mid J\right)\right|} \\
P_{H}\left(v_{\beta} \mid J\right)=\frac{H\left(v_{\beta} \mid J\right)}{\left|H\left(v_{\beta} \mid J\right)\right|}, J \in B_{2}^{2^{n}} \\
P_{H}\left(\tilde{u}_{\beta} \mid J\right)=\frac{H\left(\tilde{u}_{\beta} \mid J\right)}{\left|H\left(\tilde{u}_{\beta} \mid J\right)\right|} \\
P_{H}\left(\tilde{v}_{\beta} \mid J\right)=\frac{H\left(\tilde{v}_{\beta} \mid J\right)}{\left|H\left(\tilde{v}_{\beta} \mid J\right)\right|}, J \in B_{2}^{2^{n}}
\end{array}\right.
$$

Here, all interactive maps are also restricted in $[0,1]^{2}$ areas respectively.

Distributions are dependant on the data set as a whole and are not sensitive to varying under special sequences. Under this condition, when the data set has been exhaustively listed, then the same distributions are always linked to the given signal set.

Let $I M(J)=\left\{P_{H}(u \mid J), P_{H}(v \mid J)\right\}$ or $\left\{P_{H}(\tilde{u} \mid J), P_{H}(\tilde{v} \mid J)\right\}$ be the output results of an IM module. Then the eight histogram distributions provide invariant spectrums to represent properties among different interactive conditions.

Using such descriptions, the output results of the CEIM component are $\{C E(J), I M(J)\}$.

From a given function, a set of histograms can be generated as a group of eight probability histograms in variant phase space. Two groups of sixteen histograms are required. Sample cases are shown in Figures 3-4(I-II). 


\section{CEL $J=3$ in MPS Eight Interactive Maps}

(I) Representative patterns of Histograms for function $J$ (a-d) symmetric cases; (e-h) antisymmetric cases

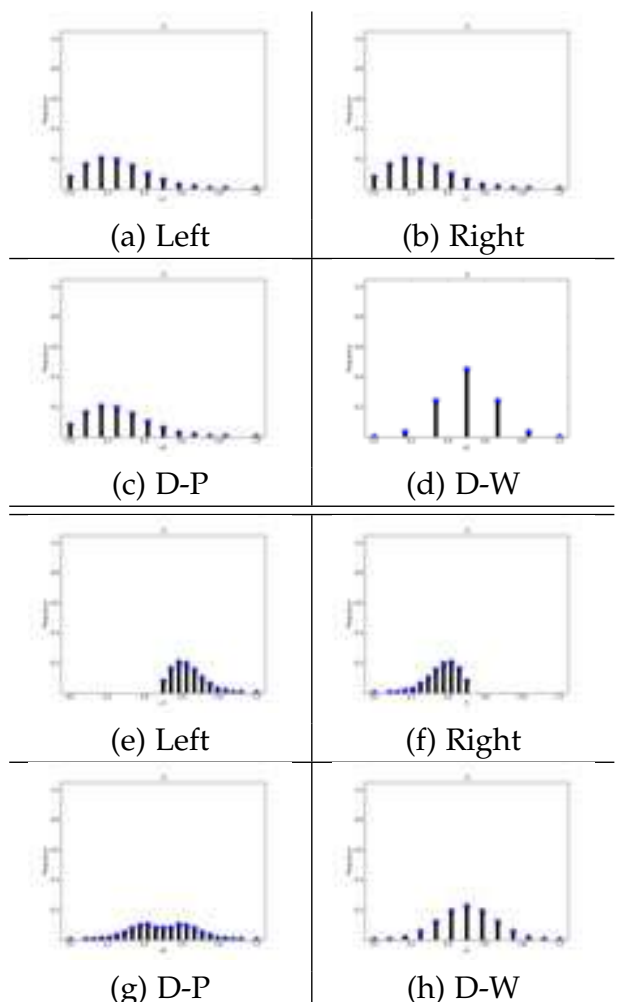

(II) $N=\{12\}, n=2, J=3$ Two groups of results in eight histograms

Figure 3. (I-II) $N=\{12\}, n=2, J=3$ Simulation results; (I) Representative Patterns for $P_{H}\left(u_{+} \mid J\right)=P_{H}\left(u_{-} \mid J\right)$ and $P_{H}\left(v_{+} \mid J\right)=P_{H}\left(1-v_{-} \mid J\right)$ conditions; (II) $N=\{12\}, n=2, J=3$ Two groups of eight interactive histograms on MPS 


\section{$P_{H}\left(\tilde{u}_{+} \mid J\right) \mid P_{H}\left(\tilde{u}_{-} \mid J\right)$

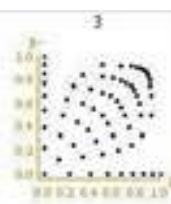 \\ (a) Left (b) Right \\ \begin{tabular}{l|l}
\hline$P_{H}\left(\tilde{u}_{0} \mid J\right)$ & $P_{H}\left(\tilde{u}_{1} \mid J\right)$
\end{tabular}

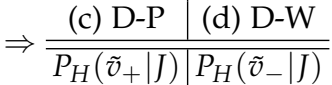 \\ \begin{tabular}{c|c} 
(e) Left & (f) Right \\
\hline$P_{H}\left(\tilde{v}_{0} \mid J\right)$ & $P_{H}\left(\tilde{v}_{1} \mid J\right)$
\end{tabular} \\ (g) D-P (h) D-W \\ CEL $J=3$ in CPS Eight Interactive Maps}

(I) Representative patterns of Histograms for function $J$ (a-d) symmetric cases; (e-h) antisymmetric cases

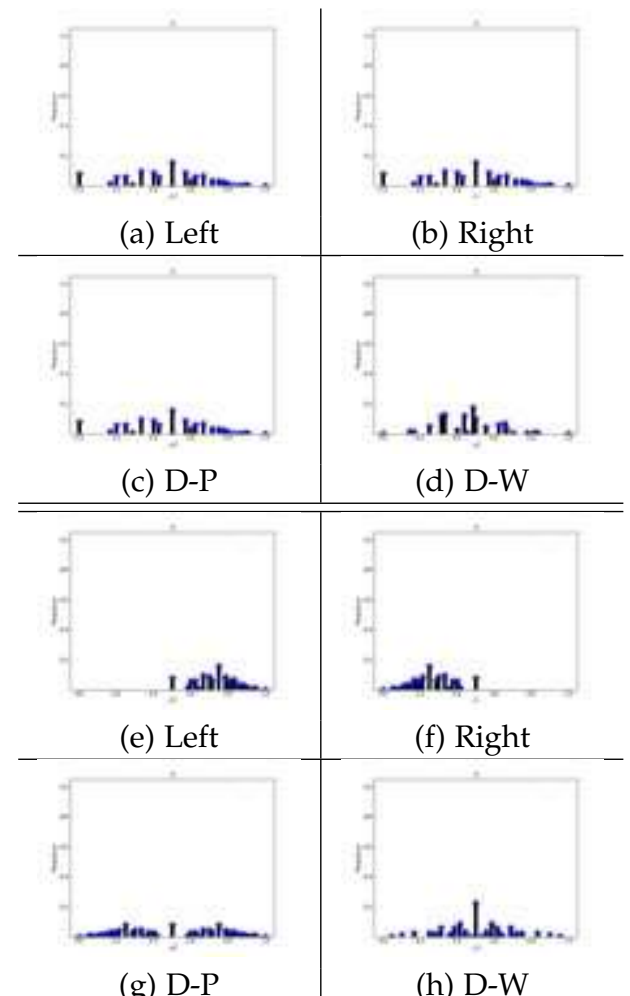

(II) $N=\{12\}, n=2, J=3$ Two groups of results in eight histograms

Figure 4. (I-II) $N=\{12\},, n=2, J=3$ Simulation results; (I) Representative Patterns for $P_{H}\left(\tilde{u}_{+} \mid J\right)=P_{H}\left(\tilde{u}_{-} \mid J\right)$ and $P_{H}\left(\tilde{v}_{+} \mid J\right)=P_{H}\left(1-\tilde{v}_{-} \mid J\right)$ conditions; (II) $N=\{12\}, n=2, J=3$ Two groups of eight interactive histograms on CPS 


\section{GEIM global ensemble and interactive map matrices}

The GEIM component is composed of two modules: SCEIM Sets of CE\&IM, and CIM CE\&IM Matrices.

$\{C E(J), I M(J)\}$ and $\forall J$ are put in the SCEIM module to generate sets of CEs and IMs on each given function exhaustively. All generated CEs and IMs are organized by the CIM module under the FC condition in which a special variant coding scheme is applied for a global configuration of output matrices.

\subsection{SCEIM sets of canonical ensembles and interactive maps}

The SCEIM module produces $\{S C E, S I M\}$ composed of all possible CE and IM sets of $\forall J$ under exhaustive conditions.

$$
\begin{aligned}
& S C E=\left\{\forall J, C E(J) \mid J \in B_{2}^{2^{n}}\right\} \\
& S I M=\left\{\forall J,\left\{P_{H}(u \mid J), P_{H}(v \mid J)\right\} \text { or }\left\{P_{H}(\tilde{u} \mid J), P_{H}(\tilde{v} \mid J)\right\} \mid J \in B_{2}^{2^{n}}\right\}
\end{aligned}
$$

Meanwhile, the SCE and the SIM provide output results to the CIM module.

\subsection{CIM canonical ensemble and interactive map matrices}

In addition to using $\{S C E, S I M\}$ as inputs, the $F C$ also inputs a code scheme to determine a detailed configuration for each matrix.

\subsubsection{Global Matrix Representations}

In the CIM module, $\{S C E, S I M\}$ inputs have nine sets of CEs and IMs. Each set is composed of $2^{2^{n}}$ elements and each element is a histogram or a plane lattice. The CIM module arranges all $2^{2^{n}}$ elements generated as a matrix by a given FC code scheme.

\subsubsection{The Matrix and Its elements}

For a given FC scheme, let $F C(J)=\left\langle J^{1} \mid J^{0}\right\rangle$, each element

$$
\left\{\begin{array}{l}
M_{\left\langle J^{1} \mid J^{0}\right\rangle}\left(u_{\beta} \mid J\right)=P_{H}\left(u_{\beta} \mid J\right) \\
M_{\left\langle J^{1} \mid J^{0}\right\rangle}\left(v_{\beta} \mid J\right)=P_{H}\left(v_{\beta} \mid J\right) \\
J \in B_{2}^{2^{n}} ; J^{1}, J^{0} \in B_{2}^{2^{n-1}}
\end{array}\right.
$$

\subsubsection{Representative patterns of matrices}

Four cases of FC codes are selected for illustrations in this Chapter. Further discussion on the details of variant coding scheme has been previously published in [40,44].

For example, four sample cases are shown in Figure 5 under relevant conditions. 


\begin{tabular}{|c|c|c|c|}
\hline 0 & 1 & 2 & 3 \\
\hline 4 & 5 & 6 & 7 \\
\hline 8 & 9 & 10 & 11 \\
\hline 12 & 13 & 14 & 15 \\
\hline
\end{tabular}

(a) SL code

\begin{tabular}{|c|c|c|c|}
\hline 0 & 8 & 1 & 9 \\
\hline 2 & 10 & 3 & 11 \\
\hline 4 & 12 & 5 & 13 \\
\hline 6 & 14 & 7 & 15 \\
\hline
\end{tabular}

(b) W code

\begin{tabular}{|c|c|c|c|}
\hline 0 & 2 & 1 & 3 \\
\hline 4 & 6 & 5 & 7 \\
\hline 8 & 10 & 9 & 11 \\
\hline 12 & 14 & 13 & 15 \\
\hline
\end{tabular}

(c) F code

\begin{tabular}{|c|c|c|c|}
\hline 0 & 4 & 1 & 5 \\
\hline 2 & 6 & 3 & 7 \\
\hline 8 & 12 & 9 & 13 \\
\hline 10 & 14 & 11 & 15 \\
\hline
\end{tabular}

(d) $\mathrm{C}$ code

Figure 5. (a-c) Four Cases of Matrix configurations for $n=2$ on FC (a) Case 1. SL code (b) Case 2. W code (c) Case 3. F code (d) Case 4. $C$ code

Case 1: $F C=\{n=2, P=(3210)\}$ a SL code;

Case 2: $F C=\{n=2, P=(2103)\}$ a $W$ code;

Case 3: $F C=\{n=2, P=(3201)\}$ a F code;

Case 4: $F C=\{n=2, P=(3102)\}$ a $C$ code.

Under each condition, each FC code is a special configuration to make sixteen elements arranged as a $4 \times 4$ matrix.

For the matrices in this chapter, four configurations are applied to construct sample matrices with elements arranged for illustration purposes.

\section{Representation model}

Figure 6 presents a graphical summary of the above. Further representations are offered in Figure 7 to show the main steps in creating a CE in the MPS or CPS and IMs relevant to global CEM and IMM procedures.

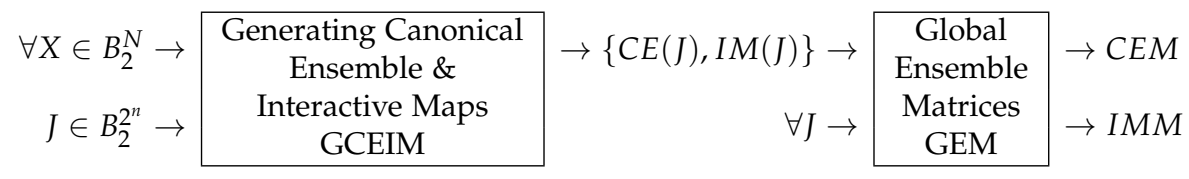

Figure 6. Diagrammatical Representation of VPS Model

\section{Symbolic representations on selected cases}

Using a representational model, for a given condition, there are two sets of CEM in both MPS and CPS. Each set contains a CEM and eight IMMs. Since each matrix contains $2^{2^{n}}$ elements, the existence of so many possible configurations adds to the difficulties in reaching a satisfactory understanding of the data sets. In this section, symbolic representations are applied to show more clearly the essential symmetric properties of various matrices. Using variant logic, the following equations can be established for an $n=2$ condition to apply $(a, b, c, d)=(10,8,2,0)$ and $(\tilde{a}, \tilde{b}, \tilde{c}, \tilde{d})=(10,14,11,15)$ for each meta function. 

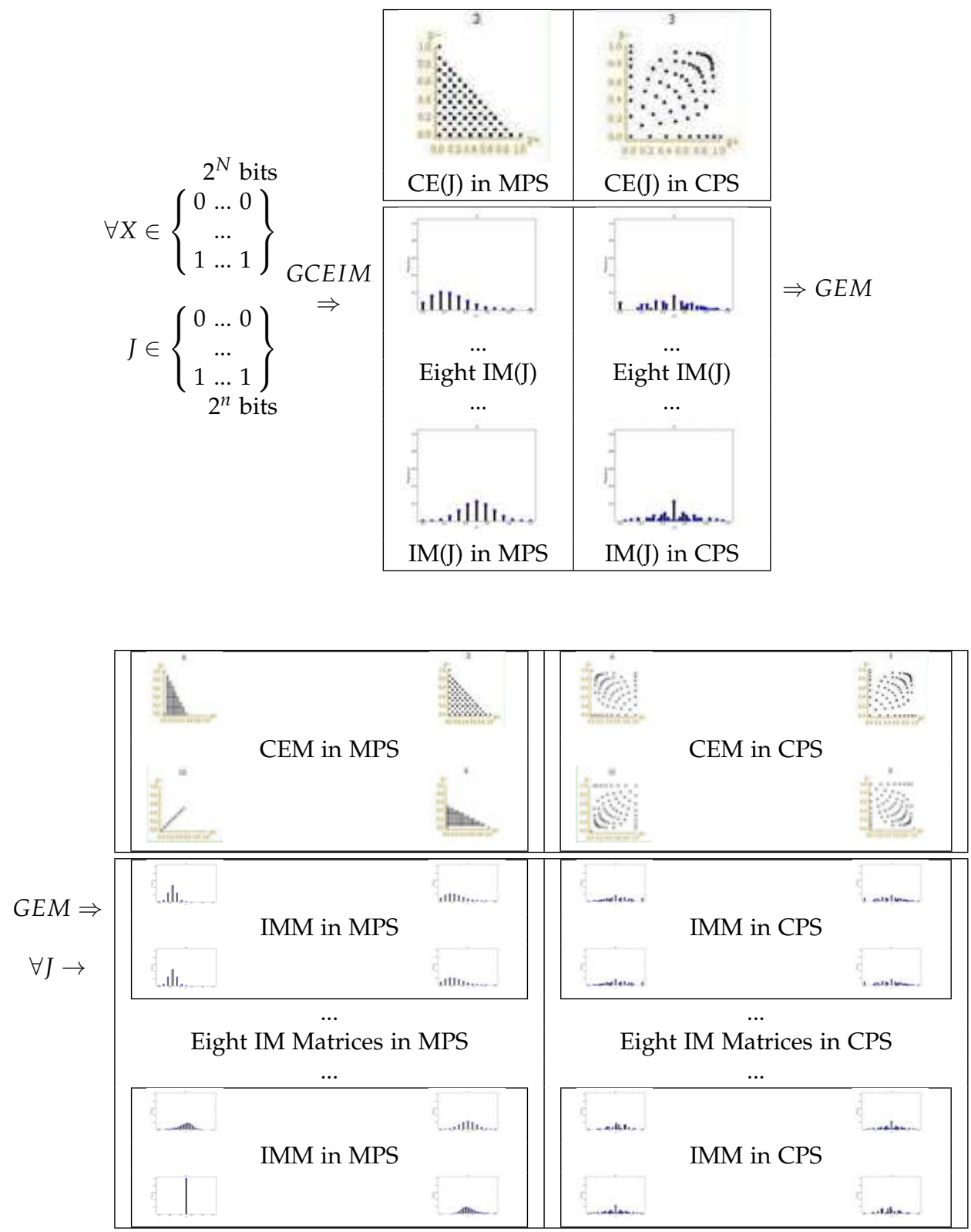

Figure 7. Illustrations of Representative Model of the VPS for GCEIM and GEM results 


$$
\left\{\begin{aligned}
0 & =\langle 0 \mid 10\rangle=\langle d \mid \tilde{a}\rangle=d ; & 1 & =\langle 0 \mid 11\rangle=\langle d \mid \tilde{c}\rangle ; \\
2 & =\langle 2 \mid 10\rangle=\langle c \mid \tilde{a}\rangle=c ; & 3 & =\langle 2 \mid 11\rangle=\langle c \mid \tilde{c}\rangle ; \\
4 & =\langle 0 \mid 14\rangle=\langle d \mid \tilde{b}\rangle ; & 5 & =\langle 0 \mid 15\rangle=\langle d \mid \tilde{d}\rangle ; \\
6 & =\langle 2 \mid 14\rangle=\langle c \mid \tilde{b}\rangle ; & 7 & =\langle 2 \mid 15\rangle=\langle c \mid \tilde{d}\rangle ; \\
8 & =\langle 8 \mid 10\rangle=\langle b \mid \tilde{a}\rangle=b ; & 9 & =\langle 8 \mid 11\rangle=\langle b \mid \tilde{c}\rangle ; \\
10 & =\langle 10 \mid 10\rangle=\langle a \mid \tilde{a}\rangle=a=\tilde{a} ; & 11 & =\langle 10 \mid 11\rangle=\langle a \mid \tilde{c}\rangle=\tilde{c} ; \\
12 & =\langle 8 \mid 14\rangle=\langle b \mid \tilde{b}\rangle ; & 13 & =\langle 8 \mid 15\rangle=\langle b \mid \tilde{d}\rangle ; \\
14 & =\langle 10 \mid 14\rangle=\langle a \mid \tilde{b}\rangle=\tilde{b} ; & 15 & =\langle 10 \mid 15\rangle=\langle a \mid \tilde{d}\rangle=\tilde{d} .
\end{aligned}\right.
$$

Using this symbolic style, four cases of configurations and their polarized decompositions are represented as follows.

Case 1: SL code $P=(3210)$

$$
\begin{aligned}
& \left(\begin{array}{cccc}
0 & 1 & 2 & 3 \\
4 & 5 & 6 & 7 \\
8 & 9 & 10 & 11 \\
12 & 13 & 14 & 15
\end{array}\right)=\left\langle\left(\begin{array}{cccc}
0 & 0 & 2 & 2 \\
0 & 0 & 2 & 2 \\
8 & 8 & 10 & 10 \\
8 & 8 & 10 & 10
\end{array}\right) \mid\left(\begin{array}{llll}
10 & 11 & 10 & 11 \\
14 & 15 & 14 & 15 \\
10 & 11 & 10 & 11 \\
14 & 15 & 14 & 15
\end{array}\right)\right\rangle \\
& =\left\langle\left(\begin{array}{llll}
d & d & c & c \\
d & d & c & c \\
b & b & a & a \\
b & b & a & a
\end{array}\right) \mid\left(\begin{array}{cccc}
\tilde{a} & \tilde{c} & \tilde{a} & \tilde{c} \\
\tilde{b} & \tilde{d} & \tilde{b} & \tilde{d} \\
\tilde{a} & \tilde{c} & \tilde{a} & \tilde{c} \\
\tilde{b} & \tilde{d} & \tilde{b} & \tilde{d}
\end{array}\right)\right\rangle
\end{aligned}
$$

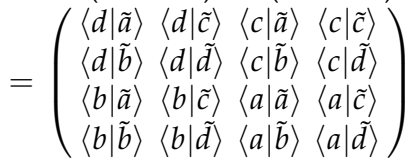

$$
\begin{aligned}
& =\langle 2 \times 2 \text { Block }| \text { Cross }\rangle \text {; }
\end{aligned}
$$

Case 2: $\mathrm{W}$ code $P=(2103)$

$$
\begin{aligned}
& \left(\begin{array}{cccc}
0 & 8 & 1 & 9 \\
2 & 10 & 3 & 11 \\
4 & 12 & 5 & 13 \\
6 & 14 & 7 & 15
\end{array}\right)=\left\langle\left(\begin{array}{cccc}
0 & 8 & 0 & 8 \\
2 & 10 & 2 & 10 \\
0 & 8 & 0 & 8 \\
2 & 10 & 2 & 10
\end{array}\right) \mid\left(\begin{array}{llll}
10 & 10 & 11 & 11 \\
10 & 10 & 11 & 11 \\
14 & 14 & 15 & 15 \\
14 & 14 & 15 & 15
\end{array}\right)\right\rangle \\
& =\left\langle\left(\begin{array}{llll}
d & b & d & b \\
c & a & c & a \\
d & b & d & b \\
c & a & c & a
\end{array}\right) \mid\left(\begin{array}{llll}
\tilde{a} & \tilde{a} & \tilde{c} & \tilde{c} \\
\tilde{b} & \tilde{b} & \tilde{d} & \tilde{d} \\
\tilde{a} & \tilde{a} & \tilde{c} & \tilde{c} \\
\tilde{b} & \tilde{b} & \tilde{d} & \tilde{d}
\end{array}\right)\right\rangle \\
& =\left(\begin{array}{ccc}
\langle d \mid \tilde{a}\rangle & \langle b \mid \tilde{a}\rangle\langle d \mid \tilde{c}\rangle\langle b \mid \tilde{c}\rangle \\
\langle c \mid \tilde{b}\rangle\langle a \mid \tilde{b}\rangle\langle c \mid \tilde{d}\rangle\langle a \mid \tilde{d}\rangle \\
\langle d \mid \tilde{a}\rangle\langle b \mid \tilde{a}\rangle\langle d \mid \tilde{c}\rangle\langle b \mid \tilde{c}\rangle \\
\langle c \mid \tilde{b}\rangle\langle a \mid \tilde{b}\rangle\langle c \mid \tilde{d}\rangle\langle a \mid \tilde{d}\rangle
\end{array}\right) \\
& =\langle\text { Cross }| 2 \times 2 \text { Block }\rangle \text {; }
\end{aligned}
$$


Case 3: F code $P=(3201)$

$$
\begin{aligned}
& \left(\begin{array}{cccc}
0 & 2 & 1 & 3 \\
4 & 6 & 5 & 7 \\
8 & 10 & 9 & 11 \\
12 & 14 & 13 & 15
\end{array}\right)=\left\langle\left(\begin{array}{cccc}
0 & 2 & 0 & 2 \\
0 & 2 & 0 & 2 \\
8 & 10 & 8 & 10 \\
8 & 10 & 8 & 10
\end{array}\right) \mid\left(\begin{array}{llll}
10 & 10 & 11 & 11 \\
14 & 14 & 15 & 15 \\
10 & 10 & 11 & 11 \\
14 & 14 & 15 & 15
\end{array}\right)\right\rangle \\
& =\left\langle\left(\begin{array}{llll}
d & c & d & c \\
d & c & d & c \\
b & a & b & a \\
b & a & b & a
\end{array}\right) \mid\left(\begin{array}{llll}
\tilde{a} & \tilde{a} & \tilde{c} & \tilde{c} \\
\tilde{b} & \tilde{b} & \tilde{d} & \tilde{d} \\
\tilde{a} & \tilde{a} & \tilde{c} & \tilde{c} \\
\tilde{b} & \tilde{b} & \tilde{d} & \tilde{d}
\end{array}\right)\right\rangle \\
& =\left(\begin{array}{cccc}
\langle d \mid \tilde{a}\rangle & \langle c \mid \tilde{a}\rangle & \langle d \mid \tilde{c}\rangle & \langle c \mid \tilde{c}\rangle \\
\langle d \mid \tilde{b}\rangle & \langle c \mid \tilde{b}\rangle & \langle d \mid \tilde{d}\rangle & \langle c \mid \tilde{d}\rangle \\
\langle b \mid \tilde{a}\rangle & \langle a \mid \tilde{a}\rangle & \langle b \mid \tilde{c}\rangle & \langle a \mid \tilde{c}\rangle \\
\langle b \mid \tilde{b}\rangle & \langle a \mid \tilde{b}\rangle & \langle b \mid \tilde{d}\rangle & \langle a \mid \tilde{d}\rangle
\end{array}\right) \\
& =\langle\mathrm{V}-2 \mathrm{Run} \mid \mathrm{H}-2 \mathrm{Run}\rangle \text {; }
\end{aligned}
$$

Case 4: $C$ code $P=(3102)$

$$
\begin{aligned}
& \left(\begin{array}{cccc}
0 & 4 & 1 & 5 \\
2 & 6 & 3 & 7 \\
8 & 12 & 9 & 13 \\
10 & 14 & 11 & 15
\end{array}\right)=\left\langle\left(\begin{array}{cccc}
0 & 0 & 0 & 0 \\
2 & 2 & 2 & 2 \\
8 & 8 & 8 & 8 \\
10 & 10 & 10 & 10
\end{array}\right) \mid\left(\begin{array}{llll}
10 & 14 & 11 & 15 \\
10 & 14 & 11 & 15 \\
10 & 14 & 11 & 15 \\
10 & 14 & 11 & 15
\end{array}\right)\right\rangle \\
& =\left\langle\left(\begin{array}{llll}
d & d & d & d \\
c & c & c & c \\
b & b & b & b \\
a & a & a & a
\end{array}\right) \mid\left(\begin{array}{llll}
\tilde{a} & \tilde{b} & \tilde{c} & \tilde{d} \\
\tilde{a} & \tilde{b} & \tilde{c} & \tilde{d} \\
\tilde{a} & \tilde{b} & \tilde{c} & \tilde{d} \\
\tilde{a} & \tilde{b} & \tilde{c} & \tilde{d}
\end{array}\right)\right\rangle
\end{aligned}
$$

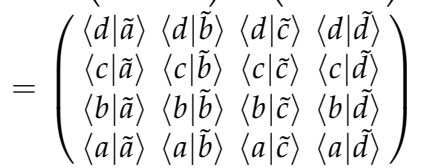

$$
\begin{aligned}
& =\langle\mathrm{H}-4 \text { Run } \mid \mathrm{V}-4 \mathrm{Run}\rangle \text {. }
\end{aligned}
$$

Six pairs $\{0: 15,1: 7,2: 11,4: 13,6: 9,8: 14\}$ of distributions may have symmetry properties

$$
\begin{aligned}
& 0: 15=\langle d \mid \tilde{a}\rangle:\langle a \mid \tilde{d}\rangle ; \\
& 1: 7=\langle d \mid \tilde{c}\rangle:\langle c \mid \tilde{d}\rangle ; \\
& 2: 11=\langle c \mid \tilde{a}\rangle:\langle a \mid \tilde{c}\rangle ; \\
& 4: 13=\langle d \mid \tilde{b}\rangle:\langle b \mid \tilde{d}\rangle ; \\
& 6: 9=\langle c \mid \tilde{b}\rangle:\langle b \mid \tilde{c}\rangle ; \\
& 8: 14=\langle b \mid \tilde{a}\rangle:\langle a \mid \tilde{b}\rangle .
\end{aligned}
$$


Six pairs $\{1: 8,2: 4,3: 12,5: 10,7: 14,11: 13\}$ of distributions may have anti-symmetry properties

$$
\begin{aligned}
1: 8 & =\langle d \mid \tilde{c}\rangle:\langle b \mid \tilde{a}\rangle ; \\
2: 4 & =\langle c \mid \tilde{a}\rangle:\langle d \mid \tilde{b}\rangle ; \\
3: 12 & =\langle c \mid \tilde{c}\rangle:\langle b \mid \tilde{b}\rangle ; \\
5: 10 & =\langle d \mid \tilde{d}\rangle:\langle a \mid \tilde{a}\rangle ; \\
7: 14 & =\langle c \mid \tilde{d}\rangle:\langle a \mid \tilde{b}\rangle ; \\
11: 13 & =\langle a \mid \tilde{c}\rangle:\langle b \mid \tilde{d}\rangle .
\end{aligned}
$$

Two pairs $\{3: 12,5: 10\}$ of distributions may have self-conjugate properties with both symmetry and anti-symmetry properties.

$$
\begin{aligned}
& 3: 12=\langle c \mid \tilde{c}\rangle:\langle b \mid \tilde{b}\rangle \\
& 5: 10=\langle d \mid \tilde{d}\rangle:\langle a \mid \tilde{a}\rangle .
\end{aligned}
$$

Four pairs $\{0: 15,3: 12,5: 10,6: 9\}$ of distributions may have special properties.

$$
\begin{aligned}
& 0: 15=\langle d \mid \tilde{a}\rangle:\langle a \mid \tilde{d}\rangle \\
& 3: 12=\langle c \mid \tilde{c}\rangle:\langle b \mid \tilde{b}\rangle ; \\
& 5: 10=\langle d \mid \tilde{d}\rangle:\langle a \mid \tilde{a}\rangle ; \\
& 6: 9=\langle c \mid \tilde{b}\rangle:\langle b \mid \tilde{c}\rangle .
\end{aligned}
$$

Regions of Measurements in MPS can be illustrated as

$$
\operatorname{MPS}:\left(\begin{array}{ccc}
0 & & \\
& \ldots & \\
& \cdots & \\
& \ldots & \\
10 & & 15
\end{array}\right) \Rightarrow\left(\begin{array}{cccc}
{[1,0]} & (-,-) & \ldots & {[1 / 2,1 / 2]} \\
(-, 0) & \ldots & & \ldots \\
\ldots & & \ldots & (-,-) \\
(0,0) & \ldots & (0,-) & {[0,1]}
\end{array}\right)
$$

Regions of Measurements in CPS can be illustrated as

$$
\text { CPS : }\left(\begin{array}{cccc}
0 & & & 5 \\
& \ldots & \\
& \ldots & \\
10 & & & 15
\end{array}\right) \Rightarrow\left(\begin{array}{cccc}
{[1,0]} & (1,-) & \ldots & {[1,1]} \\
(-, 0) & \ldots & & \ldots \\
\ldots & & \ldots & (-, 1) \\
(0,0) & \ldots & (0,-) & {[0,1]}
\end{array}\right)
$$

\section{Sample results}

\subsection{CEM groups}

Using $n=2$ configurations, relevant CEMs on either MPS or CPS are shown in Figure 8(a-h). 


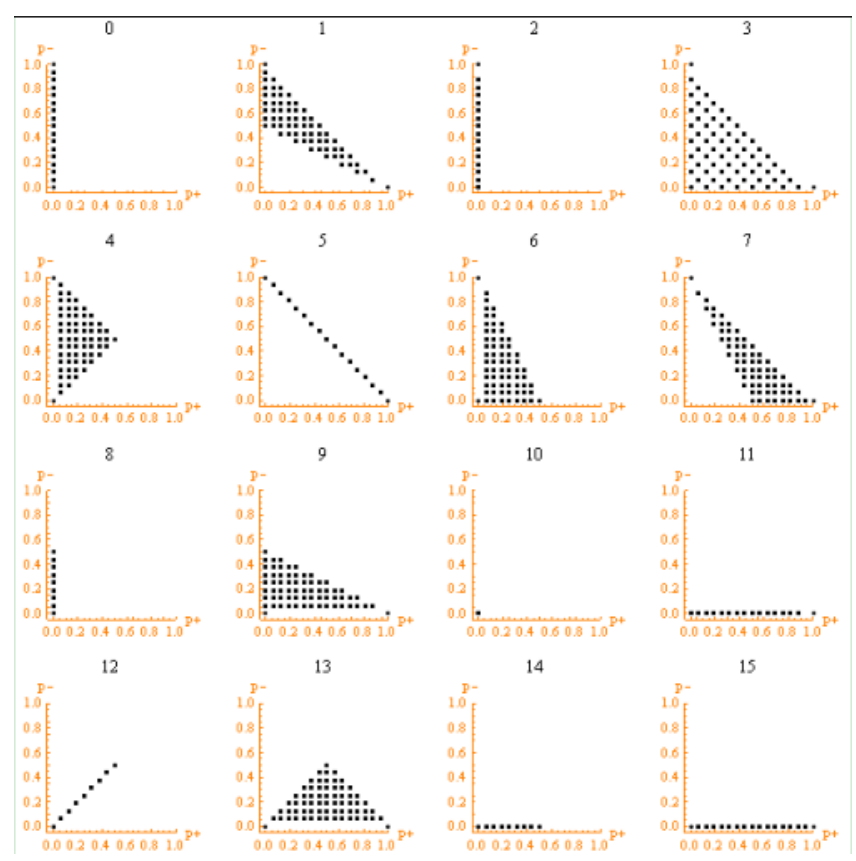

(a) SL in MPS
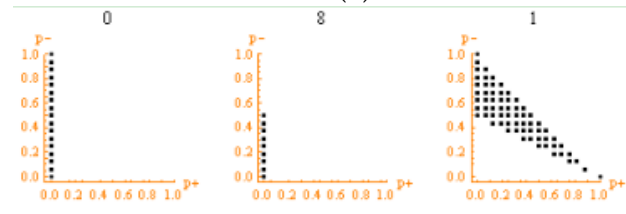

9
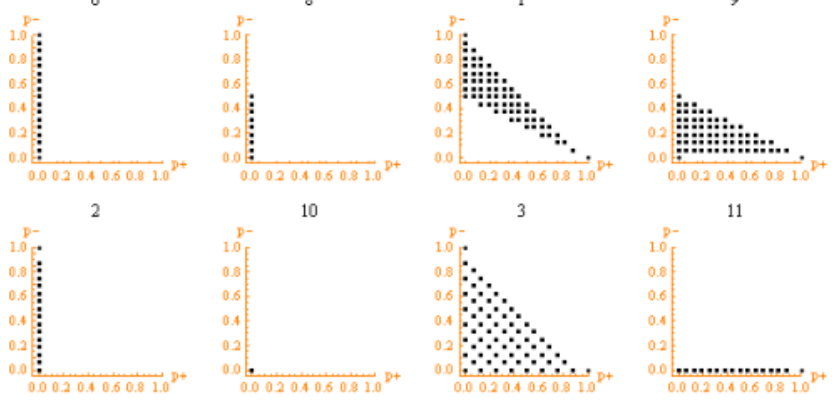

12
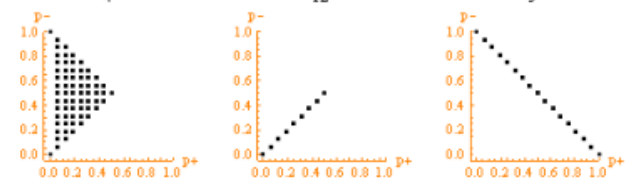

11

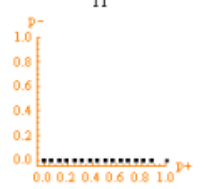

13
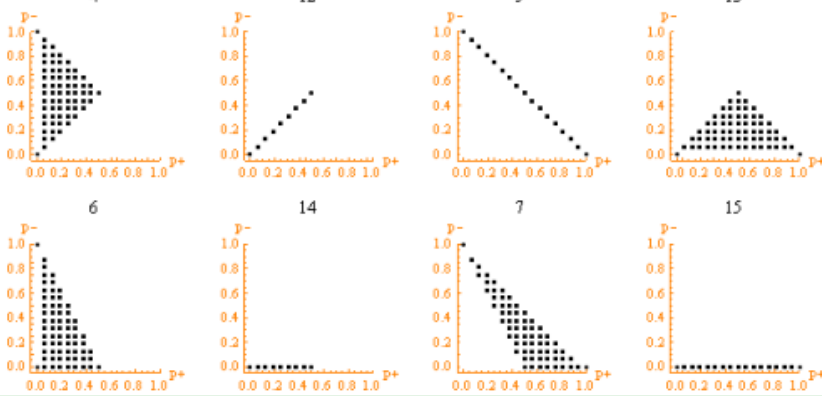

14

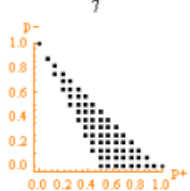

15
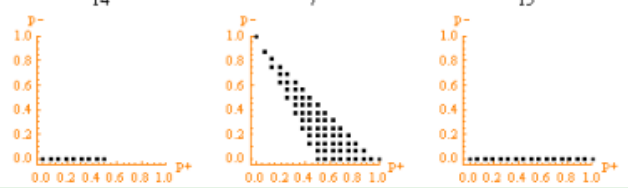

(b) W in MPS 

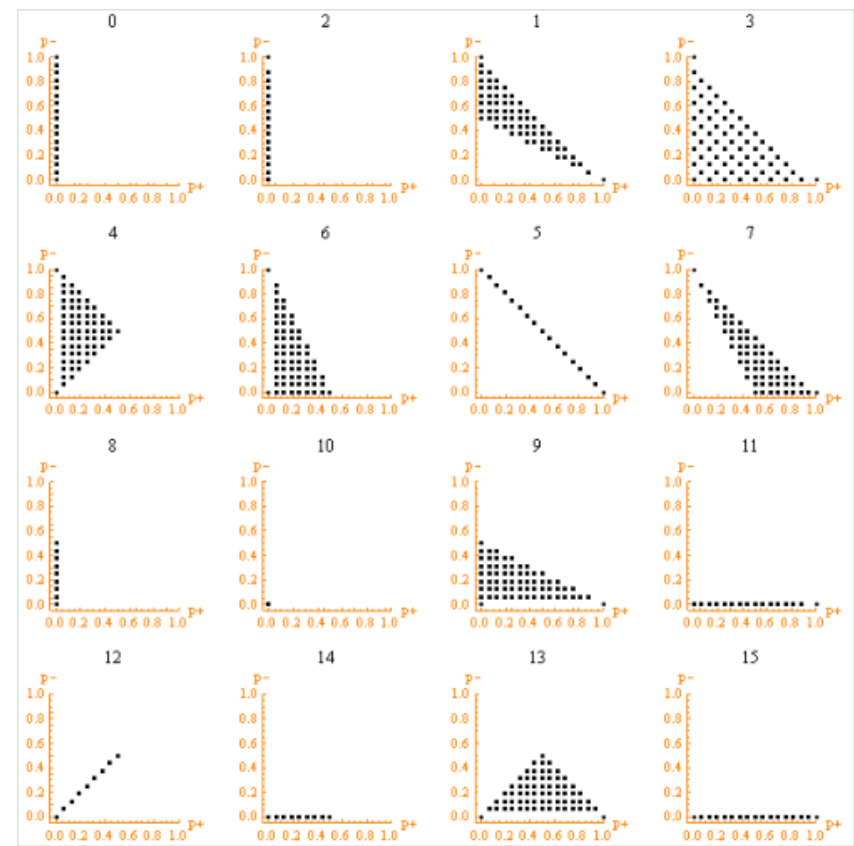

10
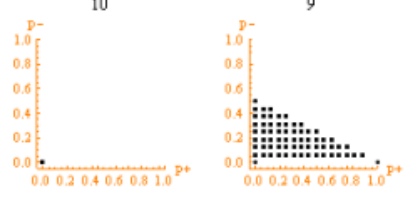

11

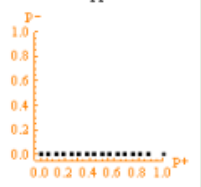

13

15
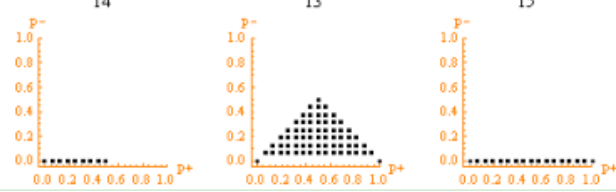

(c) F in MPS
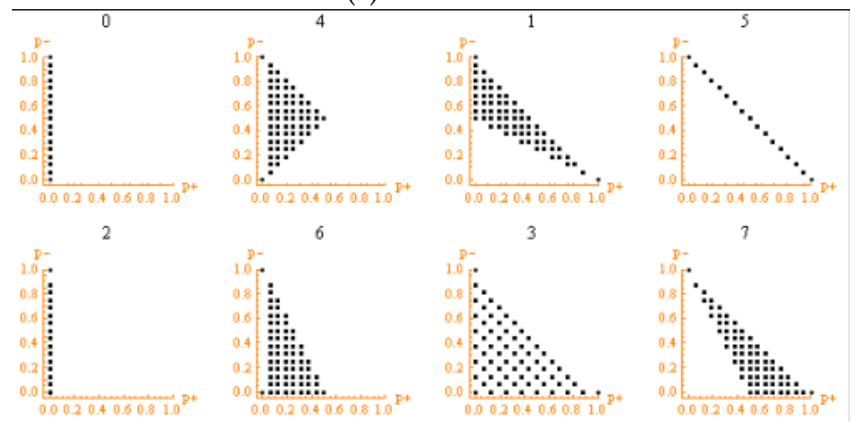

12
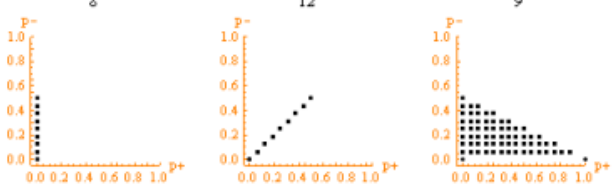

13
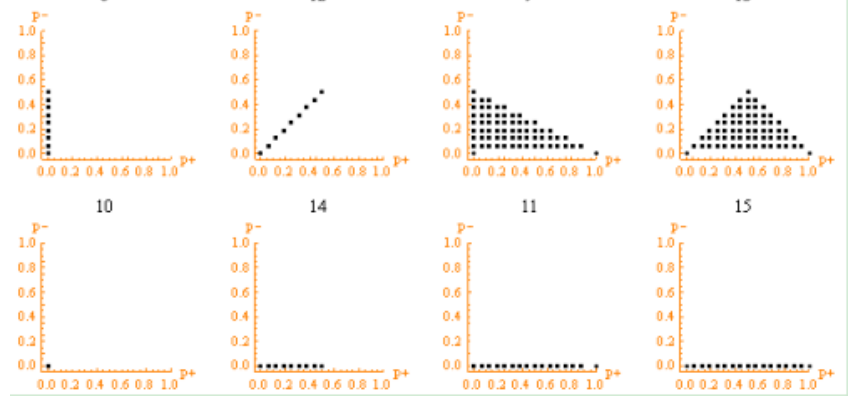

(d) C in MPS 

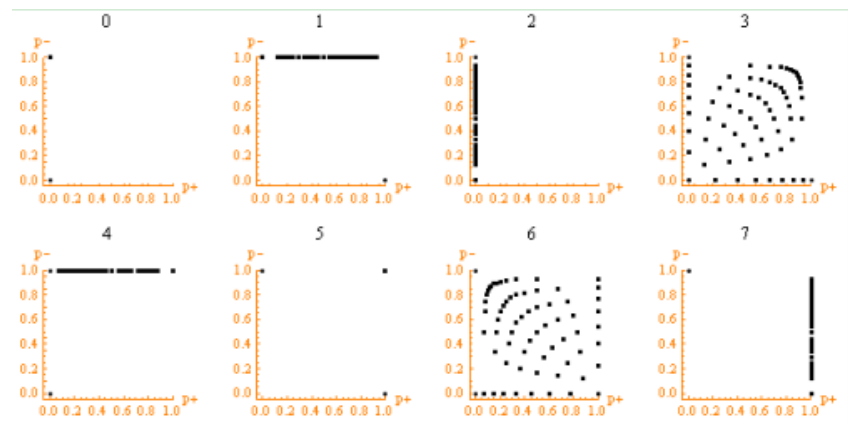

9

10

11
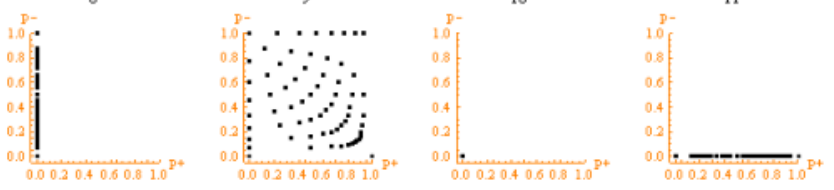

12

13

14
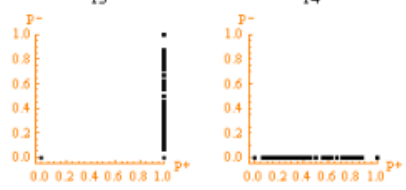

15

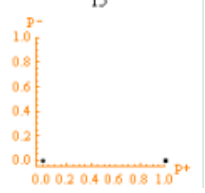

(e) SL in CPS
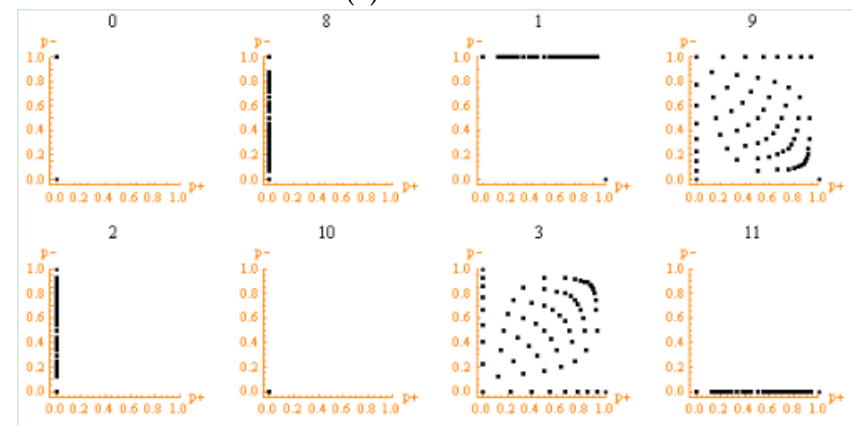

10

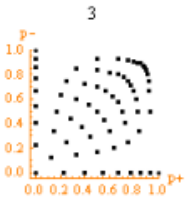

11
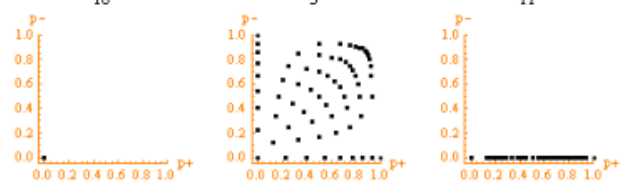

12

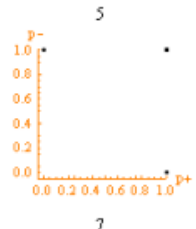

13
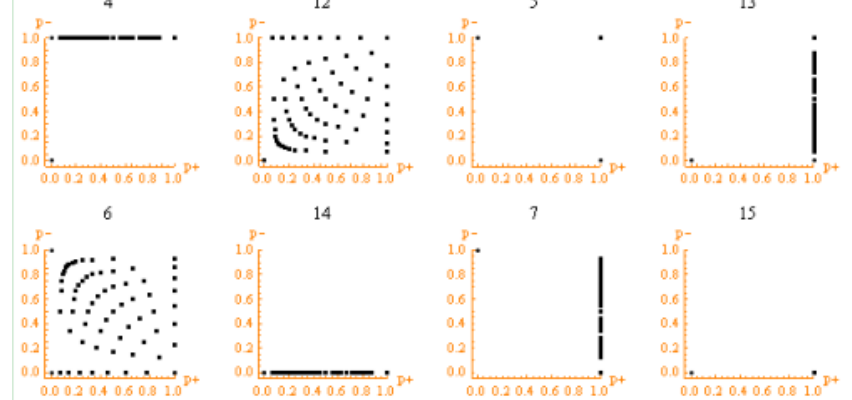

(f) $\mathrm{W}$ in CPS 


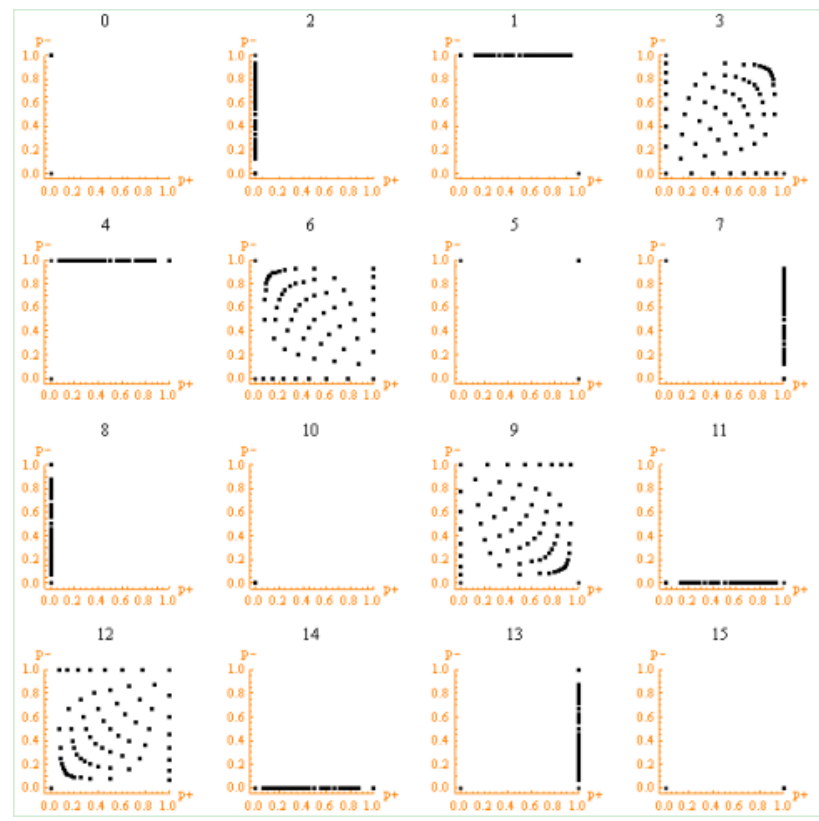

(g) F in CPS

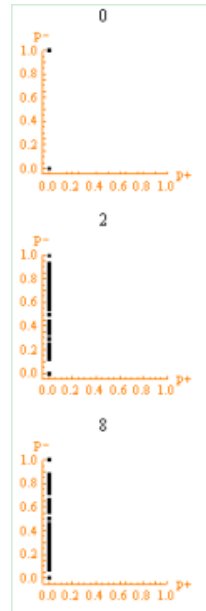

10

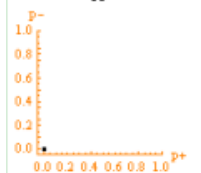

4
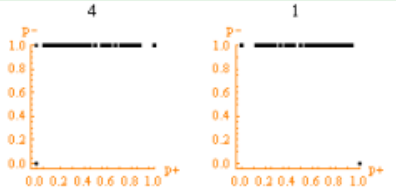

3

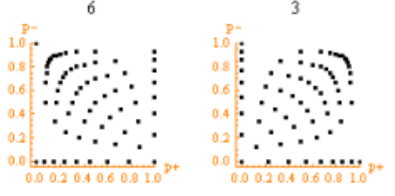

12

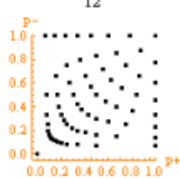

14

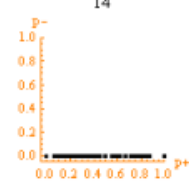

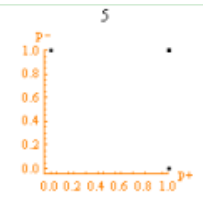

7

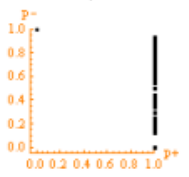

13
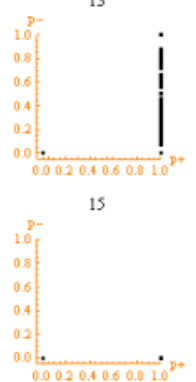

(h) $\mathrm{C}$ in CPS

15

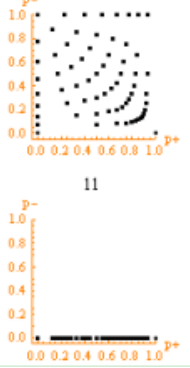

$0.00020 .40 .60 .810^{8}$

Figure 8. (a-f) Matrices of Plane Lattices of VPS for MPS and CPS in $\{S L, W, F, C\}$ codes, (a-d) MPS, (e-h) CPS; (a,e) SL code, $(b, f)$ W code, $(c, g)$ F code, (d,h) C code. 


\subsection{IMM groups}

IM Matrices under MPS are shown in Figures 9(M1-M32) and IM Matrices under CPS are shown in Figures 10(C1-C32).

\section{Analysis of visual distributions}

\subsection{VPS organization}

Two groups of matrices are shown in Figure 8. The four matrices shown as 8 (a-d) illustrate MPS conditions and the four matrices shown as $8(\mathrm{e}-\mathrm{h})$ illustrate CPS conditions. Considering various CEs exhibiting conjugate symmetry properties, such arrangements may be noted to have similar distributions along the diagonal and anti-diagonal directions so that it is possible to find a pair of CEs with each CE pair-matched by a geometric transformation to another $\mathrm{CE}$ through either rotation or reflection.

\subsubsection{MPS Structures}

The four CE matrices in the MPS group as shown in Figure 8(a-d) can be analyzed as follows.

\section{SL in MPS}

For the matrix in Figure 8(a) showing SL in MPS, 16 CEs are arranged in linear order from $0-15$ in the $4 \times 4$ matrix. Only two pairs of $\{0: 15,6: 9\}$ CEs have conjugate symmetry properties.

\section{$\mathrm{W}$ in MPS}

For the matrix in Figure 8(b) showing W in MPS, 16 CEs are not arranged in linear order in $\{0, \ldots, 15\}$ in the $4 \times 4$ matrix. Only two pairs of $\{0: 15,6: 9\}$ CEs have conjugate symmetry properties.

\section{$F$ in MPS}

However, for the matrix in Figure 8(c) showing F in MPS, 16 CEs exhibit more conjugate pairs in the $4 \times 4$ matrix. Here, six pairs of CEs $\{0: 15,1: 7,2: 11,4: 13,6: 9,8: 14\}$ show conjugate symmetry properties.

\section{$\mathrm{C}$ in MPS}

Also, for the matrix in Figure 8(d) showing $C$ in MPS, we find the same number of conjugate pairs as with the F condition. Moreover, not only do six pairs of CEs \{0:15, 1:7, 2:11, 4:13, $6: 9,8: 14\}$ exhibit conjugate symmetry properties, but also four CEs $\{10,8,2,0\}$ are polarized on the vertical as per the left column and four CEs $\{10,14,11,15\}$ are polarized on the horizontal direction as per the bottom row. In addition, nine CEs showing interactive properties are evident top-right in a $3 \times 3$ matrix.

\subsubsection{CPS Structures}

The four CE matrices shown in Figure 8(e-h) in the CPS group can be analyzed as follows.

\section{SL in CPS}


For the matrix in Figure 8(e) showing SL in CPS, 16 CEs are arranged in linear order from $0-15$ as a $4 \times 4$ matrix. Four pairs of CEs $\{0: 15,3: 12,5: 10,6: 9\}$ have conjugate symmetry properties.

\section{$W$ in CPS}

For the matrix in Figure 8(f) showing W in CPS, 16 CEs are not arranged in linear order. However, four pairs of $C E s\{0: 15,3: 12,5: 10,6: 9\}$ have conjugate symmetry properties.

\section{$F$ in CPS}

For the matrix in Figure 8(g) showing F in CPS, we can recognize two CE groups where each group has six pairs of CEs with conjugate symmetry properties $\{0: 15,1: 7,2: 11,4: 13,6: 9,8: 14\}$ and $\{2: 4,1: 8,3: 12,5: 10,7: 14,11: 13\}$.

\section{$\mathrm{C}$ in CPS}

For the matrix in Figure 8(h) showing C in CPS, 12 CEs (out of 16) show conjugate pairing. This is the same number of conjugate pairs as is evident with the F condition in CPS. Also, if we look at polarization, the matrix for C in CPS is very different from the other coding matrices. It has significant polarized properties connecting the outer elements of the matrix. Here, four CEs $\{10,8,2,0\}$ are polarized on the vertical as per the left column, and another four CEs $\{5,7,13,15\}$ as per the right column. Also, four CEs $\{0,4,1,8\}$ are polarized on the horizontal as per the top-row and another four CEs $\{10,14,11,15\}$ as per the bottom-row. Four more CEs $\{3,6,9,12\}$ exhibit interactive properties in a $2 \times 2$ central grid. In all, five distinct regions can be identified as significant. It is interesting to note such remarkable symmetry illustrating interactions between and among these meta functions.

\subsection{IMM organization}

From one matrix in VPS, eight matrices corresponding to the two vectors $\{u, v\}=$ $\left\{\left(u_{+}, u_{-}, u_{0}, u_{1}\right),\left(v_{+}, v_{-}, v_{0}, v_{1}\right)\right\}$ can be generated showing interactive properties under symmetry/anti-symmetry, and synchronous/asynchronous conditions respectively. A total of 64 matrices are shown in two groups in Figures 9 (M1-M32) for MPS and in Figures 10 (C1-C32) for CPS, respectively.

\subsubsection{MPS Structures}

\section{SL group in MPS}

For the SL group in Figure 9 (M1-M8), the two matrix vectors $\{u, v\}=$ $\left\{\left(u_{+}, u_{-}, u_{0}, u_{1}\right),\left(v_{+}, v_{-}, v_{0}, v_{1}\right)\right\}$ are best considered separately.

M1-M4: Let us first consider elements M1-M4 where the four matrices of $\left(u_{+}, u_{-}, u_{0}, u_{1}\right)$ are in a symmetry group,

In $u_{+}$matrix M1, elements in the columns and rows are arranged in what may be described as a periodic crossing structure.

In $u_{-}$matrix M2, four elements with the same IMs are arranged in a $2 \times 2$ block with four distinct distributions being observed. 
In $u_{0}$ matrix $\mathrm{M} 3$, each element shows simple additions from elements in $u_{+}$and $u_{-}$ respectively. It it interesting to note that only two pairs of positions $\{0: 15,6: 9\}$ are similarly distributed in the relevant MPS matrix.

However, in $u_{1}$ matrix M4, significant symmetry properties can be observed. Four pairs $\{0: 15,3: 12,5: 10,6: 9\}$ have symmetry or anti-symmetry properties that are different from the $u_{0}$ condition.

M4-M8: Let us now consider elements M5-M8 where the four matrices of $\left(v_{+}, v_{-}, v_{0}, v_{1}\right)$ are in an anti-symmetry group,

In $v_{+}$matrix M5, elements in the columns and rows are arranged as periodic crossing structures.

In $v_{-}$matrix M6, four elements with the same IMs are arranged in a $2 \times 2$ block with four distinct distributions observed.

In $v_{0}$ matrix $\mathrm{M} 7$, each element shows simple additions from elements in $v_{+}$and $v_{-}$ respectively. Only two pairs of positions $\{0: 15,6: 9\}$ are similarly distributed in the relevant MPS matrix.

In $v_{1}$ matrix M8, significant symmetry properties can be observed. Two pairs $\{0: 15,6: 9\}$ have anti-symmetry properties that are the same as the $v_{0}$ condition.

\section{W group in MPS}

For the $\mathrm{W}$ group in Figure 9 (M9-M16), the two matrix vectors $\{u, v\}=$ $\left\{\left(u_{+}, u_{-}, u_{0}, u_{1}\right),\left(v_{+}, v_{-}, v_{0}, v_{1}\right)\right\}$ are best considered separately.

M9-M12: Let us now consider elements M9-M12 where the four matrices $\left(u_{+}, u_{-}, u_{0}, u_{1}\right)$ are in a symmetry group,

In $u_{+}$matrix M9, four elements with the same IMs are arranged in a $2 \times 2$ block with four distinct distributions observed.

In $u_{-}$matrix M10, elements in the columns and rows are arranged as a periodic crossing structure.

In $u_{0}$ matrix M11, each element shows simple additions with elements in $u_{+}$and $u_{-}$ respectively. It it interesting to note that only two pairs of positions $\{0: 15,6: 9\}$ are similarly distributed in the relevant MPS matrix.

However, in $u_{1}$ matrix M12, significant symmetry properties can be observed. Four pairs $\{0: 15,3: 12,5: 10,6: 9\}$ have symmetry or anti-symmetry properties that are different from the $u_{0}$ condition.

M13-M16: Let us now consider elements M13-M16 where the four matrices $\left(v_{+}, v, v_{0}, v_{1}\right)$ are in an anti-symmetry group,

In $v_{+}$matrix M13, four elements with the same IMs are arranged in a $2 \times 2$ block with four distinct distributions observed.

In $v_{-}$matrix M14, elements in the columns and rows are arranged as a periodic crossing structure. 
In $v_{0}$ matrix M15, each element shows simple additions with elements in $v_{+}$and $v_{-}$ respectively. Only two pairs of positions $\{0: 15,6: 9\}$ are similarly distributed in the relevant MPS matrix.

In $v_{1}$ matrix M16, significant symmetry properties can be observed. Two pairs $\{0: 15,6: 9\}$ have anti-symmetry properties the same as under the $v_{0}$ condition.

\section{F group in MPS}

For the $\mathrm{F}$ group in Figure 9 (M17-M24), the two matrix vectors $\{u, v\}=$ $\left\{\left(u_{+}, u_{-}, u_{0}, u_{1}\right),\left(v_{+}, v_{-}, v_{0}, v_{1}\right)\right\}$ are best considered separately.

M17-M20: Let us now consider elements M17-M20 where the four matrices $\left(u_{+}, u_{-}, u_{0}, u_{1}\right)$ are in a symmetry group,

In $u_{+}$matrix M17, the horizontal elements are arranged in $\mathrm{H}-2 \mathrm{R}$ patterns and vertical elements are in a periodic crossing structure.

In $u_{-}$matrix M18, vertical elements are arranged in $\mathrm{V}-2 \mathrm{R}$ patterns and the horizontal elements as a periodic crossing structure.

In $u_{0}$ matrix M19, each element shows simple additions with elements in $u_{+}$and $u_{-}$ respectively. It it interesting to note that six pairs of positions $\{0: 15,1: 7$. 2:11, 4:13, 6:9, $8: 14\}$ are similarly distributed.

However, in $u_{1}$ matrix M20, significant symmetry properties can be observed. Not only do six pairs $\{0: 15,1: 7$. 2:11, 4:13, 6:9, 8:14\} have symmetry properties and another six pairs of $\{1: 8,2: 4,3: 12,5: 10,7: 14,11: 13\}$ with anti-symmetry properties but there are also significantly differences compared with the $u_{0}$ condition.

M21-M24: Let us now consider elements M17-M20 where the four matrices of $\left(v_{+}, v, v_{0}, v_{1}\right)$ are in an anti-symmetry group,

In $v_{+}$matrix M21, the horizontal elements are arranged in $\mathrm{H}-2 \mathrm{R}$ patterns and the vertical elements as a periodic crossing structure.

In $v_{-}$matrix M22, the vertical elements are arranged in $\mathrm{V}-2 \mathrm{R}$ patterns and the horizontal elements as a periodic crossing structure.

In $v_{0}$ matrix M23, each element shows simple additions with elements in $v_{+}$and $v_{-}$ respectively. It it interesting to note that six pairs of positions $\{0: 15,1: 7$. 2:11, 4:13, 6:9, $8: 14\}$ are in the anti-symmetry distribution.

In $v_{1}$ matrix M24, significant symmetry properties can be observed. Six pairs $\{0: 15,1: 7$. $2: 11,4: 13,6: 9,8: 14\}$ have anti-symmetry properties and two pairs $\{2: 4,11: 13\}$ have symmetry properties.

\section{$C$ group in MPS}

For the $\mathrm{C}$ group in Figure 9 (M25-M32), two matrix vectors $\{u, v\}=$ $\left\{\left(u_{+}, u_{-}, u_{0}, u_{1}\right),\left(v_{+}, v_{-}, v_{0}, v_{1}\right)\right\}$ are best considered separately.

M25-M28: Let us now consider elements M25-M28 where the four matrices of $\left(u_{+}, u_{-}, u_{0}, u_{1}\right)$ are in a symmetry group, 
In $u_{+}$matrix M25, the horizontal elements are in a periodic crossing structure and the vertical elements are arranged in V-4R patterns

In $u_{-}$matrix M26, the horizontal elements are arranged in $\mathrm{H}-4 \mathrm{R}$ patterns and the vertical elements as a periodic crossing structure.

In $u_{0}$ matrix M27, each element shows simple additions with elements in $u_{+}$and $u_{-}$ respectively. It it interesting to note that six pairs of positions $\{0: 15,1: 7$. 2:11, 4:13, 6:9, $8: 14\}$ are similarly distributed.

However, in $u_{1}$ matrix M28, significant symmetry properties can be observed. Not only do six pairs $\{0: 15,1: 7.2: 11,4: 13,6: 9,8: 14\}$ have symmetry but another six pairs $\{1: 8,2: 4,3: 12,5: 10$, $7: 14,11: 13\}$ have anti-symmetry properties, all significantly different from the $u_{0}$ condition.

M29-M32: Let us now consider elements M29-M32 where the four matrices of $\left(v_{+}, v, v_{0}, v_{1}\right)$ are in an anti-symmetry group,

In $v_{+}$matrix M29, the horizontal elements are arranged in $H-4 R$ patterns and the vertical elements as a periodic crossing structure.

In $v_{-}$matrix $\mathrm{M} 30$, the horizontal elements are arranged in $\mathrm{H}-4 \mathrm{R}$ patterns and the vertical elements as a periodic crossing structure.

In $v_{0}$ matrix M31, each element shows simple additions with elements in $v_{+}$and $v_{-}$ respectively. The distribution of six pairs of positions $\{0: 15,1: 7$. 2:11, 4:13, 6:9, 8:14\} exhibit anti-symmetry.

In $v_{1}$ matrix $M 32$, significant symmetry properties can be observed. Six pairs $\{0: 15,1: 7$. $2: 11,4: 13,6: 9,8: 14\}$ have anti-symmetry properties and two pairs $\{2: 4,11: 13\}$ have symmetry properties.

\subsubsection{CPS Structures}

Four groups of different configurations shown in Figure 10 (C1-C32) are discussed separately as follows.

\section{SL group in CPS}

For the SL group in Figure 10 (C1-C8), he two matrix vectors $\{\tilde{u}, \tilde{v}\}=$ $\left\{\left(\tilde{u}_{+}, \tilde{u}_{-}, \tilde{u}_{0}, \tilde{u}_{1}\right),\left(\tilde{v}_{+}, \tilde{v}_{-}, \tilde{v}_{0}, \tilde{v}_{1}\right)\right\}$ are best considered separately.

C1-C4: Let us now consider elements C1-C4 where the four matrices of $\left(\tilde{u}_{+}, \tilde{u}_{-}, \tilde{u}_{0}, \tilde{u}_{1}\right)$ are in a symmetry group,

In $\tilde{u}_{+}$matrix $\mathrm{C} 1$, elements in the columns and rows are in a periodic crossing structure.

In $\tilde{u}_{-}$matrix $\mathrm{C} 2$, four elements with the same IMs are arranged in a $2 \times 2$ block with four distinct distributions observed.

In $\tilde{u}_{0}$ matrix C3, each element shows simple additions from elements in $\tilde{u}_{+}$and $\tilde{u}_{-}$ respectively. It it interesting to note that four pairs of positions $\{0: 15,3: 12,5: 10,6: 9\}$ are similarly distributed in the relevant CPS matrix.

In $\tilde{u}_{1}$ matrix $C 4$, similar symmetry properties can be observed. Four pairs $\{0: 15,3: 12,5: 10$, 6:9\} have symmetry or anti-symmetry properties that are the same as for the $\tilde{u}_{0}$ condition. 
C5-C8: Let us now consider elements C5-C8 where the four matrices of $\left(\tilde{v}_{+}, \tilde{v}, \tilde{v}_{0}, \tilde{v}_{1}\right)$ are in an anti-symmetry group,

In $\tilde{v}_{+}$matrix $\mathrm{C} 5$, elements in the columns and rows are arranged in a periodic crossing structure.

In $\tilde{v}_{-}$matrix C6, four elements with same IMs are arranged in a $2 \times 2$ block and four distinct distributions are observed.

In $\tilde{v}_{0}$ matrix C7, each element shows simple additions from elements in $\tilde{v}_{+}$and $\tilde{v}_{-}$ respectively. Only two pairs of positions $\{0: 15,6: 9\}$ are in the same distribution in similar arrangements.

In $\tilde{v}_{1}$ matrix $C 8$, similar symmetry properties can be observed. Four pairs $\{0: 15,3: 12,5: 10$, $6: 9\}$ have anti-symmetry properties significantly different from those in the $\tilde{v}_{0}$ condition.

\section{$\mathrm{W}$ group in CPS}

For the $\mathrm{W}$ group in Figure 10 (C9-C16), the two matrix vectors $\{\tilde{u}, \tilde{v}\}=$ $\left\{\left(\tilde{u}_{+}, \tilde{u}_{-}, \tilde{u}_{0}, \tilde{u}_{1}\right),\left(\tilde{v}_{+}, \tilde{v}_{-}, \tilde{v}_{0}, \tilde{v}_{1}\right)\right\}$ are best considered separately.

C9-C12: Let us now consider elements C9-C12 where the four matrices of $\left(\tilde{u}_{+}, \tilde{u}_{-}, \tilde{u}_{0}, \tilde{u}_{1}\right)$ are in a symmetry group,

In $\tilde{u}_{+}$matrix C9, four elements with the same IMs are arranged in a $2 \times 2$ block and four distinct distributions are observed.

In $\tilde{u}_{-}$matrix $\mathrm{C} 10$, elements in the columns and rows are arranged as a periodic crossing structure.

In $\tilde{u}_{0}$ matrix $\mathrm{C} 11$, each element shows simple additions from elements in $\tilde{u}_{+}$and $\tilde{u}_{-}$ respectively. It it interesting to note that four pairs of positions $\{0: 15,3: 12,5: 10,6: 9\}$ are distributed in similar arrangements in the relevant CPS matrix.

In $\tilde{u}_{1}$ matrix $C 12$, similar symmetry properties can be observed. Four pairs $\{0: 15,3: 12,5: 10$, $6: 9\}$ have symmetry or anti-symmetry properties that are the same as under the $\tilde{u}_{0}$ condition.

C13-C16: Let us now consider elements C13-C16 where the four matrices of $\left(\tilde{v}_{+}, \tilde{v}, \tilde{v}_{0}, \tilde{v}_{1}\right)$ are in an anti-symmetry group,

In $\tilde{v}_{+}$matrix $\mathrm{C} 13$, four elements with the same IMs are arranged in a $2 \times 2$ block and four distinct distributions are observed.

In $\tilde{v}_{-}$matrix $\mathrm{C} 14$, elements in the columns and rows are arranged as a periodic crossing structure.

In $\tilde{v}_{0}$ matrix $\mathrm{C} 15$, each element shows simple additions from elements in $\tilde{v}_{+}$and $\tilde{v}_{-}$ respectively. Only two pairs of positions $\{0: 15,6: 9\}$ show the same distribution in similar arrangements.

In $\tilde{v}_{1}$ matrix C16, significant symmetry properties can be observed. Four pairs $\{0: 15,3: 12$, $5: 10,6: 9\}$ have anti-symmetry properties that are different from those under $\tilde{v}_{0}$ conditions.

\section{$F$ group in CPS}


For the $\mathrm{F}$ group in Figure 10 (C17-C24), the two matrix vectors $\{\tilde{u}, \tilde{v}\}=$ $\left\{\left(\tilde{u}_{+}, \tilde{u}_{-}, \tilde{u}_{0}, \tilde{u}_{1}\right),\left(\tilde{v}_{+}, \tilde{v}_{-}, \tilde{v}_{0}, \tilde{v}_{1}\right)\right\}$ are best considered separately.

C17-C20: Let us now consider elements C17-C20 where the four matrices of $\left(\tilde{u}_{+}, \tilde{u}_{-}, \tilde{u}_{0}, \tilde{u}_{1}\right)$ are in a symmetry group,

In $\tilde{u}_{+}$matrix $\mathrm{C} 17$, horizontal elements are arranged in $\mathrm{H}-2 \mathrm{R}$ patterns and vertical elements are in a periodic crossing structure.

In $\tilde{u}_{-}$matrix $\mathrm{C} 18$, vertical elements are arranged in $\mathrm{V}-2 \mathrm{R}$ patterns and horizontal elements as a periodic crossing structure.

In $\tilde{u}_{0}$ matrix $\mathrm{C} 19$, each element shows simple additions from elements in $\tilde{u}_{+}$and $\tilde{u}_{-}$ respectively. It interesting to note that six pairs of positions $\{0: 15,1: 7.2: 11,4: 13,6: 9,8: 14\}$ and $\{0: 15,1: 7.2: 11,4: 13,6: 9,8: 14\}$ are in the similar distributions.

In $\tilde{u}_{1}$ matrix C20, similar symmetry properties can be observed. Not only do six pairs $\{0: 15$, 1:7. 2:11, 4:13, 6:9, 8:14\} have symmetry but also another six pairs $\{1: 8,2: 4,3: 12,5: 10,7: 14$, 11:13 \} exhibit anti-symmetry properties that are the same as under $\tilde{u}_{0}$ conditions.

C21-C24: Let us now consider elements C21-C24 where the four matrices of $\left(\tilde{v}_{+}, \tilde{v}, \tilde{v}_{0}, \tilde{v}_{1}\right)$ are in an anti-symmetry group,

In $\tilde{v}_{+}$matrix $\mathrm{C} 21$, horizontal elements are arranged in $\mathrm{H}-2 \mathrm{R}$ patterns and vertical elements as periodic crossing structures.

In $\tilde{v}_{-}$matrix $C 22$, vertical elements are arranged in $\mathrm{V}-2 \mathrm{R}$ patterns and horizontal elements as a periodic crossing structure.

In $\tilde{v}_{0}$ matrix C23, each element shows simple additions from elements in $\tilde{v}_{+}$and $\tilde{v}_{-}$ respectively. It is interesting to note that only six pairs of positions $\{0: 15,1: 7$. 2:11, 4:13, $6: 9,8: 14\}$ show anti-symmetry distributions.

In $\tilde{v}_{1}$ matrix C24, significant symmetry properties can be observed. Six pairs $\{0: 15,1: 7.2: 11$, $4: 13,6: 9,8: 14\}$ have anti-symmetry properties and six pairs $\{1: 8,2: 4,3: 12,5: 10,7: 14,11: 13\}$ have symmetry properties.

\section{C group in CPS}

For the $\mathrm{C}$ group in Figure 10 (C25-C32), the two matrix vectors $\{\tilde{u}, \tilde{v}\}=$ $\left\{\left(\tilde{u}_{+}, \tilde{u}_{-}, \tilde{u}_{0}, \tilde{u}_{1}\right),\left(\tilde{v}_{+}, \tilde{v}_{-}, \tilde{v}_{0}, \tilde{v}_{1}\right)\right\}$ are best considered separately.

C25-C28: Let us now consider elements C25-C28 where the four matrices of $\left(\tilde{u}_{+}, \tilde{u}_{-}, \tilde{u}_{0}, \tilde{u}_{1}\right)$ are in a symmetry group,

In $\tilde{u}_{+}$matrix $\mathrm{C} 25$, horizontal elements are arranged as a periodic crossing structure. and vertical elements are arranged in $\mathrm{V}-4 \mathrm{R}$ patterns

In $\tilde{u}_{-}$matrix $\mathrm{C} 26$, horizontal elements are arranged in $\mathrm{H}-4 \mathrm{R}$ patterns and vertical elements as a periodic crossing structure.

In $\tilde{u}_{0}$ matrix C27, each element shows simple additions from elements in $u_{+}$and $u_{-}$ respectively. It is interesting to note that six pairs of positions $\{0: 15,1: 7$. 2:11, 4:13, 6:9, 8:14 $\}$ are similarly distributed and six pairs of positions $\{1: 8,2: 4,3: 12,5: 10,7: 14,11: 13\}$ show anti-symmetry properties. 


\begin{tabular}{cc|c|c|c|l}
\hline Type & Case & CP $\in$ MPS & CP $\in$ CPS & GP & Notes \\
\hline SL & $\mathrm{P}=(3210)$ & $2(a)$ & $4(a, d)$ & N & Limited conjugate symmetry \\
W & $\mathrm{P}=(2103)$ & $2(a)$ & $4(a, d)$ & N & Limited conjugate symmetry \\
F & $\mathrm{P}=(3201)$ & $6(e)$ & $12(e, f)$ & N & Pairs conjugate symmetry \\
C & $\mathrm{P}=(3102)$ & $6(e)$ & $12(e, f)$ & Y & Global symmetry \\
\hline
\end{tabular}

Table 4. Global Symmetry Properties on CE Matrices

In $\tilde{u}_{1}$ matrix C28, significant symmetry properties can be observed. Not only do six pairs $\{0: 15,1: 7$. 2:11, 4:13, 6:9, 8:14\} show symmetry but also another six pairs $\{1: 8,2: 4,3: 12,5: 10$, $7: 14,11: 13\}$ exhibit anti-symmetry properties similar to those under $\tilde{u}_{0}$ conditions.

C29-C32: Let us now consider elements C29-C32 where the four matrices of $\left(\tilde{v}_{+}, \tilde{v}, \tilde{v}_{0}, \tilde{v}_{1}\right)$ are in an anti-symmetry group,

In $\tilde{v}_{+}$matrix $\mathrm{C} 29$, horizontal elements are arranged in $\mathrm{H}-4 \mathrm{R}$ patterns and vertical elements are arranged as a periodic crossing structure.

In $\tilde{v}_{-}$matrix $\mathrm{C} 30$, horizontal elements are arranged in $\mathrm{H}-4 \mathrm{R}$ patterns and vertical elements are arranged as a periodic crossing structure.

In $\tilde{v}_{0}$ matrix $\mathrm{C} 31$, each element shows simple additions from elements in $\tilde{v}_{+}$and $\tilde{v}_{-}$ respectively. Two pairs of positions $\{0: 15,4: 13,6: 9,8: 14\}$ exhibit anti-symmetry distributions.

In $\tilde{v}_{1}$ matrix C32, significant symmetry properties can be observed. Six pairs $\{0: 15,1: 7$. 2:11, 4:13, 6:9, 8:14\} have anti-symmetry properties and another six pairs $\{1: 8,2: 4,3: 12,5: 10,7: 14$, 11:13 \} have symmetry properties that are different from those under $\tilde{v}_{0}$ condition.

\section{Global symmetric properties}

Working from four sets of CEM and IMM results, key global symmetry properties are presented and summarized in Table 4 for CEMs and in Table 5 for IMMs as follows.

Where $\mathrm{CP}$ is a conjugate pair, GP is global polarization and a:(0:15,6:9), d:(3:12,5:10), e:(0:15,1:7,2:11,4:13,6:9,8:14), f:(1:8,2:4,3:12,5:10,7:14,11:13), are pair functions.

It is interesting to note that significant differences in symmetry properties between MPS and CPS can be observed for CEM conjugate pairs.

In general, we find double the number of incidences of symmetry properties with CPS compared with MPS shown in Table 4.

Where SP is a Symmetric Pair, ASP is an Anti-symmetric Pair, GS is Global Symmetry and a:(0:15,6:9), b:(0:15,6:9,3:12), c:(5:10), d:(3:12,5:10), e:(0:15,1:7,2:11,4:13,6:9,8:14), $\mathrm{f:}(1: 8,2: 4,3: 12,5: 10,7: 14,11: 13)$, g:(2:4,11:13) are pair functions.

It is interesting to note that symmetry properties evident in IMM groups in Table 5 are more refined than the original configurations under MPS and CPS conditions.

The classification of different projections and polarized properties can be further refined to show their various interactive activities in relevant sub-categories. Further details for conjugate pairs can be distinguished under symmetry/anti-symmetry and synchronous/asynchronous configurations. Conjugate pairs can be further differentiated as being either symmetric or anti-symmetric pairs. 


\begin{tabular}{|c|c|c|c|c|c|c|c|c|}
\hline Type & Case & Left & Right & SP(D-P) & ASP(D-P) & SP(D-W) & ASP(D-W) & GS \\
\hline$\overline{\mathrm{SL}}$ & $\begin{array}{c}\mathrm{P}=(3210) \\
u \\
v \\
\tilde{u} \\
\tilde{v}\end{array}$ & Cross & 2x2Block & $\begin{array}{c}2 \text { (a) } \\
0 \\
2(a) \\
0\end{array}$ & $\begin{array}{c}0 \\
2 \text { (a) } \\
2 \text { (d) } \\
2 \text { (a) }\end{array}$ & $\begin{array}{l}3(\mathrm{~b}) \\
0 \\
2(\mathrm{a}) \\
2 \text { (d) }\end{array}$ & $\begin{array}{l}1(\mathrm{c}) \\
2(\mathrm{a}) \\
2(\mathrm{~d}) \\
2(\mathrm{a})\end{array}$ & Weak \\
\hline W & $\begin{array}{c}\mathrm{P}=(2103) \\
u \\
v \\
\tilde{u} \\
\tilde{v}\end{array}$ & 2x2Block & Cross & $\begin{array}{c}2 \text { (a) } \\
0 \\
2(a) \\
0\end{array}$ & $\begin{array}{c}0 \\
2(\mathrm{a}) \\
2 \text { (d) } \\
2 \text { (a) }\end{array}$ & $\begin{array}{l}3 \text { (b) } \\
0 \\
2(a) \\
2(d)\end{array}$ & $\begin{array}{l}1(\mathrm{c}) \\
2(\mathrm{a}) \\
2(\mathrm{~d}) \\
2(\mathrm{a})\end{array}$ & Weak \\
\hline & $\begin{array}{c}\mathrm{P}=(3201) \\
u \\
v \\
\tilde{u} \\
\tilde{v}\end{array}$ & $\mathrm{~V}-2 \mathrm{R}$ & $\mathrm{H}-2 \mathrm{R}$ & $\begin{array}{c}6(\mathrm{e}) \\
0 \\
6(\mathrm{e}) \\
0 \\
\end{array}$ & $\begin{array}{c}0 \\
6(\mathrm{e}) \\
6(\mathrm{f}) \\
6(\mathrm{e})\end{array}$ & $\begin{array}{l}6(\mathrm{e}) \\
2(\mathrm{~g}) \\
6(\mathrm{e}) \\
6(\mathrm{e}) \\
\end{array}$ & $\begin{array}{l}6(\mathrm{f}) \\
6(\mathrm{e}) \\
6(\mathrm{f}) \\
6(\mathrm{f}) \\
\end{array}$ & Stronger \\
\hline $\mathrm{C}$ & $\begin{array}{c}\mathrm{P}=(3102) \\
u \\
v \\
\tilde{u} \\
\tilde{v}\end{array}$ & V-4R & $\mathrm{H}-4 \mathrm{R}$ & $\begin{array}{c}6(\mathrm{e}) \\
0 \\
6(\mathrm{e}) \\
0\end{array}$ & $\begin{array}{c}0 \\
6(\mathrm{e}) \\
6(\mathrm{f}) \\
6(\mathrm{e})\end{array}$ & $\begin{array}{l}6(\mathrm{e}) \\
2(\mathrm{~g}) \\
6(\mathrm{e}) \\
6(\mathrm{e})\end{array}$ & $\begin{array}{l}6(f) \\
6(\mathrm{e}) \\
6 \text { (f) } \\
6 \text { (f) }\end{array}$ & Strongest \\
\hline
\end{tabular}

Table 5. Global Symmetry Properties on IM Matrices

\subsection{Comparison of variant phase space and statistical mechanics}

Both Maxwell-Boltzmann and Darwin-Fowler schemes are considered suitable for processing isolated systems. Meanwhile, a Gibbs scheme can be applied to several different systems namely, an isolated system on a micro canonical ensemble, a closed system on a canonical ensemble, and an open system on a grand canonical ensemble [20, 23, 24, 31, 33]. Such significant differences can offer useful comparisons when considering Variant Phase Space.

Using Variant Phase Space (VPS) components and key properties of Classical Statistical Mechanics (CSM), two types of systems are compared in Table 6.

Table 6 shows some key differences that may be distinguished between VPS and CSM. Both approaches use parameters $\{n, N, X\}$ on a selected function. However, there is a distinct difference for ME with a split into non-interactive and interactive activities between Maxwell-Boltzmann on ME(VPS) and Gibbs on IP(VPS), respectively. This difference is further distinguished on CE(VPS) and IM(VPS) levels.

Normally statistical mechanics is not based on all possible functions Instead, one function with the most probable properties is selected. Only the Maxwell demon mechanism provides any possible function for potential applications, under such restriction, modern statistical mechanics has no computational mechanism for GEM capacities.

GEM capacities do not cover a Gibbs grand canonical ensemble. However, using a given configuration of variant logic function to arrange full sets of distributions similar to variation, functional capacities can be associated with a truly large number of configurations: $2^{n} ! \times 2^{2^{n}}$. This provides an opportunity to exhaust distributions for possible functions on a scale that goes way beyond the conventional framework of modern statistical mechanics. 


\begin{tabular}{|c|c|c|c|c|}
\hline Component & VPS & Meaning & CSM & Notes \\
\hline Parameter & $\begin{array}{c}n \\
N \\
X \\
J \\
\left\{p_{+}, p_{-}\right\}\end{array}$ & $\begin{array}{l}\text { Variables } \\
\text { Dimension } \\
\text { Nbit vector } \\
X \in B_{2}^{N} \\
n \text {-function } \\
J \in B_{2}^{2^{n}} \\
\text { Probability pairs } \\
\text { of measurements }\end{array}$ & $\begin{array}{c}\text { Local unit } \\
\text { Dimension } \\
\text { Random events } \\
\text { Probable function } \\
\qquad\{q, p\}\end{array}$ & $\begin{array}{r}\text { Cell unit on rule space } \\
\text { Vector Dimension on value space } \\
\text { I/O vectors } \\
\text { Selected function } \\
\text { Conjugate pairs } \\
\text { of measurements }\end{array}$ \\
\hline CME & $\begin{array}{l}\text { VM } \\
\text { PM } \\
\text { ME } \\
\text { IP }\end{array}$ & $\begin{array}{l}\text { Variant Measures } \\
\text { Probability } \\
\text { Measurements } \\
\text { Micro } \\
\text { Ensemble } \\
\text { Interactive } \\
\text { Projections } \\
\end{array}$ & $\begin{array}{c}\text { Classes of events } \\
\text { Density } \\
\text { Probability } \\
\text { Phase Point } \\
\text { Maxwell-Boltzmann } \\
\text { Micro Canonical } \\
\text { Ensemble Gibbs } \\
\end{array}$ & $\begin{array}{r}\text { Types of vector elements } \\
\text { Probability } \\
\text { on each class } \\
\text { Unit in Phase Space for } \\
\text { non interaction } \\
\text { Unit in Ensemble } \\
\text { with interaction }\end{array}$ \\
\hline CEIM & $\begin{array}{l}\text { CE } \\
\text { IM }\end{array}$ & $\begin{array}{l}\text { Canonical } \\
\text { Ensemble } \\
\text { Interactive } \\
\text { Maps }\end{array}$ & $\begin{array}{c}\text { Canonical Ensemble } \\
\text { Maxwell-Boltzmann } \\
\text { Canonical Ensemble } \\
\text { Gibbs }\end{array}$ & $\begin{array}{r}\text { Non-interactive } \\
\text { distribution } \\
\text { Interactive } \\
\text { distributions } \\
\end{array}$ \\
\hline GEM & $\begin{array}{l}\text { SCEIM } \\
\text { CIM }\end{array}$ & $\begin{array}{l}\text { Sets of CE\&IM } \\
\text { CE\&IM Matrices }\end{array}$ & $\begin{array}{c}\text { Full set of } \\
\text { Maxwell demons } \\
\text { Global distribution } \\
\text { Matrices }\end{array}$ & $\begin{array}{r}\text { Full set of } \\
\text { possible distributions } \\
\text { Matrices for non-interactive } \\
\text { and interactive distributions }\end{array}$ \\
\hline
\end{tabular}

Table 6. Comparison between VPS and CSM

\begin{tabular}{|c|c|c|c|c|}
\hline Key & Output & Operation & Strategy & Expression \\
\hline SCEIM & $\begin{array}{l}\text { Matrices for } \\
\text { CE\&IMs } \\
\text { Sets of } \\
\text { CE\&IMs }\end{array}$ & $\begin{array}{c}\text { Global organization of } \\
\text { distributions for a configuration } \\
\text { Global Integration on } \\
\text { distributions for all functions }\end{array}$ & $\begin{array}{c}\Downarrow \\
\text { Down }\end{array}$ & $\begin{array}{r}\text { Hilbert space, Dynamic } \\
\text { systems, Variation functional } \\
\text { Top-down } \\
\text { Meta distribution, distribution } \\
\text { function, periodic distribution }\end{array}$ \\
\hline 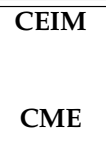 & CE\&IMs & $\begin{array}{l}\text { Integration of distributions } \\
\text { for a function } \\
\text { From local measures to } \\
\text { micro ensemble and projections }\end{array}$ & $\begin{array}{c}\text { UP } \\
\text { 介 } \\
\text { Bottom }\end{array}$ & $\begin{array}{r}\text { Maxwell-Boltzmann, Gibbs, } \\
\text { Euler, Canonical ensemble } \\
\text { Bottom-up } \\
\text { Hamilton,Lagrange, Uncertainty, } \\
\text { Fourier pairs, Phase point }\end{array}$ \\
\hline
\end{tabular}

Table 7. Operation, strategy and expression of VPS

\subsection{Corresponding structures on variant phase space}

Top-down and bottom-up strategies can both be applied to Variant Phase Space. See Table 7.

Top-down and bottom-up strategies can each open a window through which to glimpse the mysteries of Variant Phase Space. Such glimpses do not yet provide a complete picture and further investigation is clearly required.

\section{Main results}

It is appropriate to present the results as a series of detailed propositions and predictions as follows. 
For an $n$ variable function $J \in B_{2}^{2^{n}}$ and an $N$ bit vector $X \in B_{2}^{N}$, following propositions can be established.

\subsection{Propositions}

Proposition 11.1: Two types of probability measurements, Multiple and Conditional probabilities determine two distinct phase spaces, MPS and CPS.

Proof: In a PM module, multiple probabilities generates MPS and conditional probabilities create CPS.

Proposition 11.2: Two types of operations: symmetry/anti-symmetry and synchronous/asynchronous generate eight interactive projections.

Proof: Two pairs of measurement vectors $\{u, v\}$ or $\{\tilde{u}, \tilde{v}\}$ are involved in projections, where $u=\left(u_{+}, u_{-}, u_{0}, u_{1}\right), v=\left(v_{+}, v_{-}, v_{0}, v_{1}\right)$ and $\tilde{u}=\left(\tilde{u}_{+}, \tilde{u}_{-}, \tilde{u}_{0}, \tilde{u}_{1}\right), \tilde{v}=\left(\tilde{v}_{+}, \tilde{v}_{-}, \tilde{v}_{0}, \tilde{v}_{1}\right)$, each pair has eight interactive projections.

Proposition 11.3: Following a bottom-up approach, two CE and 16 IMs can be generated to exhaust all $2^{N}$ input vectors for the relevant ME and IP measurements.

Proof: Results may be generated using a CEIM module and Proposition 11.3 is further supported by Propositions 11.1 to 11.2.

Proposition 11.4: Each CE is a statistical distribution and each IM corresponds to one of eight IP modes.

Proof: A pair of probability measurements has one fixed CE combination and each IP mode corresponds to one IM distribution.

Proposition 11.5: Both Proposition 11.3 and Proposition 11.4 provide a general Maxwell Demon mechanism.

Proof: For any function, CE and IMs can be fully and exhaustively generated without reference to thermodynamic issues.

Proposition 11.6: Exhausting $\forall J \in B_{2}^{2^{n}}$, two sets of $\{C E\}$ and 16 sets of $\{\mathrm{IM}\}$ can be generated, each set contains $2^{2^{n}}$ elements and each element is a distribution.

Proof: Using the SCEIM module, they are natural outputs.

Proposition 11.7: In a variant logic framework, there are $2^{n} ! \times 2^{2^{n}}$ configurations for arranging a set of $\{C E\}$ and eight sets of $\{\mathrm{IM}\}$ into a CEM and eight IMMs.

Proof: Since each IMM has the same organization as the CEM, a total of $2^{n} ! \times 2^{2^{n}}$ configurations can be distinguished and each configuration corresponds to a variant logic matrix.

Proposition 11.8: With a top-down approach, either a CEM or an IMM on a proper configuration can be composed of two polarized matrices. Each polarized matrix has periodic structures on its columns and/or rows.

Proof: Since a proper configuration is based on $n$ periodic meta vectors and their combinations, its relative arrangements are invariant under permutation and complementary operations on the vector with $2^{2^{n}}$ bits that determine each polarized structure. 
Proposition 11.9: For MPS on C code conditions, a pair of measurements in a CEM can be arranged in a square with corners having values $\{[0,0],[1,0],[1 / 2,1 / 2],[0,1]\}$.

Proof: Under a C code configuration, the possible regions of measurements for a CEM in MPS can be shown in

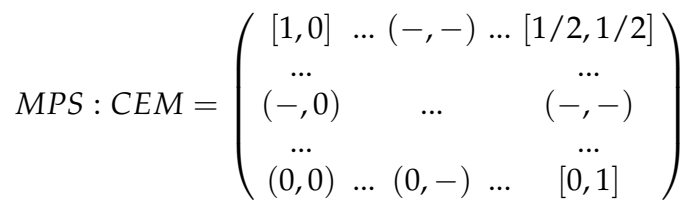

Proposition 11.10: For CPS on C code conditions, a pair of measurements in a CEM can be arranged in a square with corners having values $\{[0,0],[1,0],[1,1],[0,1]\}$.

Proof: Under a C code configuration, the possible regions of measurements for a CEM in CPS can be shown in

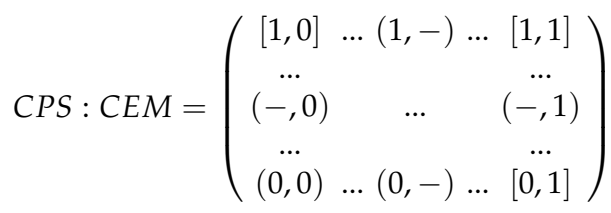

\subsection{Predictions}

Prediction 11.1: Following a bottom-up strategy, it is not possible to determine CE properties using limited numbers of ME.

This prediction points towards a more general intrinsic restriction on uncertainty effects for incomplete procedures applied to random events.

Prediction 11.2: For a configuration that is not in a variant logic framework, there may be a square integral configuration capable of providing an approximate solution.

Periodic matrices could play a key role as core components of approximation procedures.

Prediction 11.3: A sound statistical interpretation of quantum mechanics can be established using VPS construction.

Since both top-down and bottom-up strategies are included, further exploration is feasible.

Prediction 11.4: VPS construction can provide a foundation based on logic and hierarchies of measurement levels for complex dynamic systems, statistical mechanics, and cellular automata.

Through VPS construction clearly offers significant potential, this prediction needs to be tested by solid experimental and theoretical results backed by evidence. 

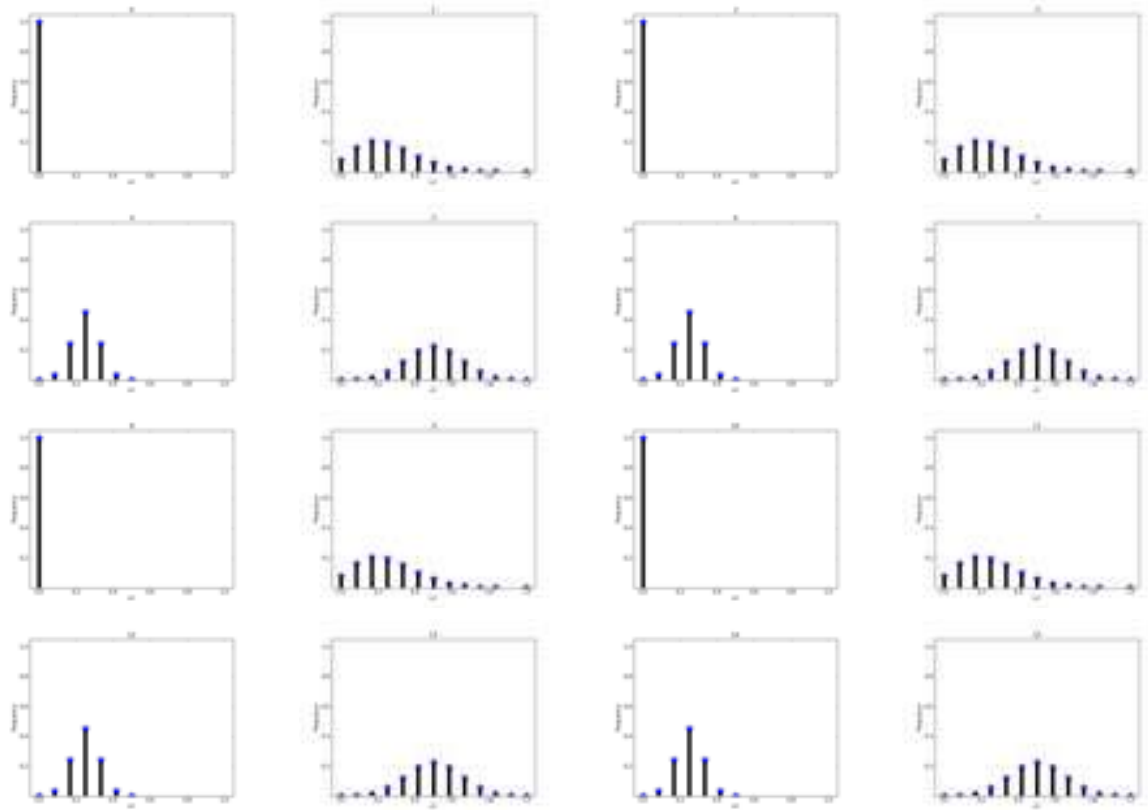

(M1) $u_{+}$SL group
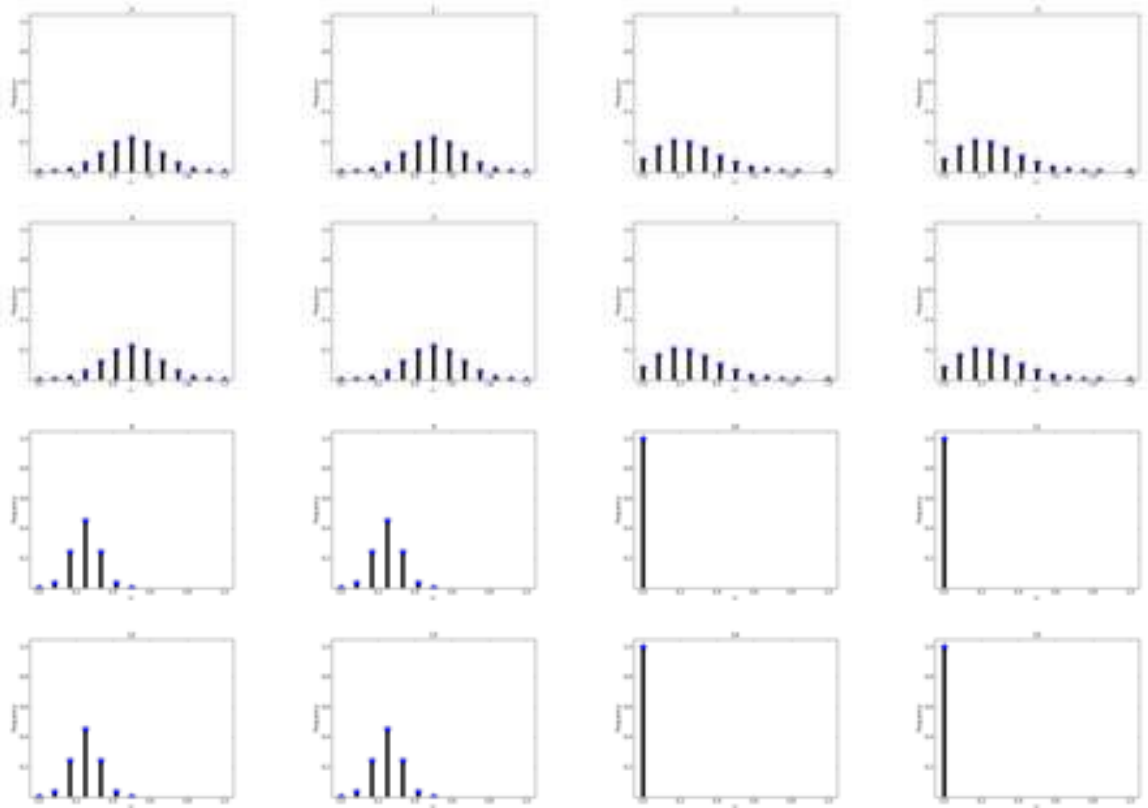

(M2) $u_{-}$SL group 

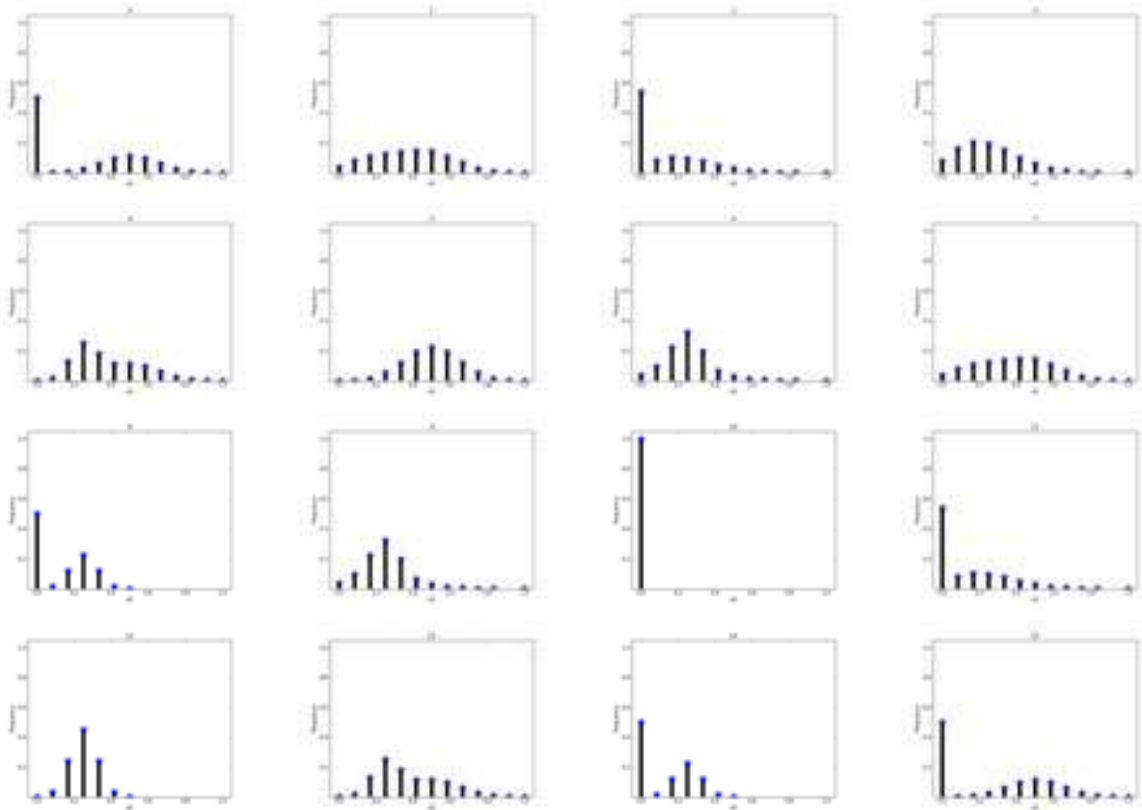

(M3) $u_{0}$ SL group
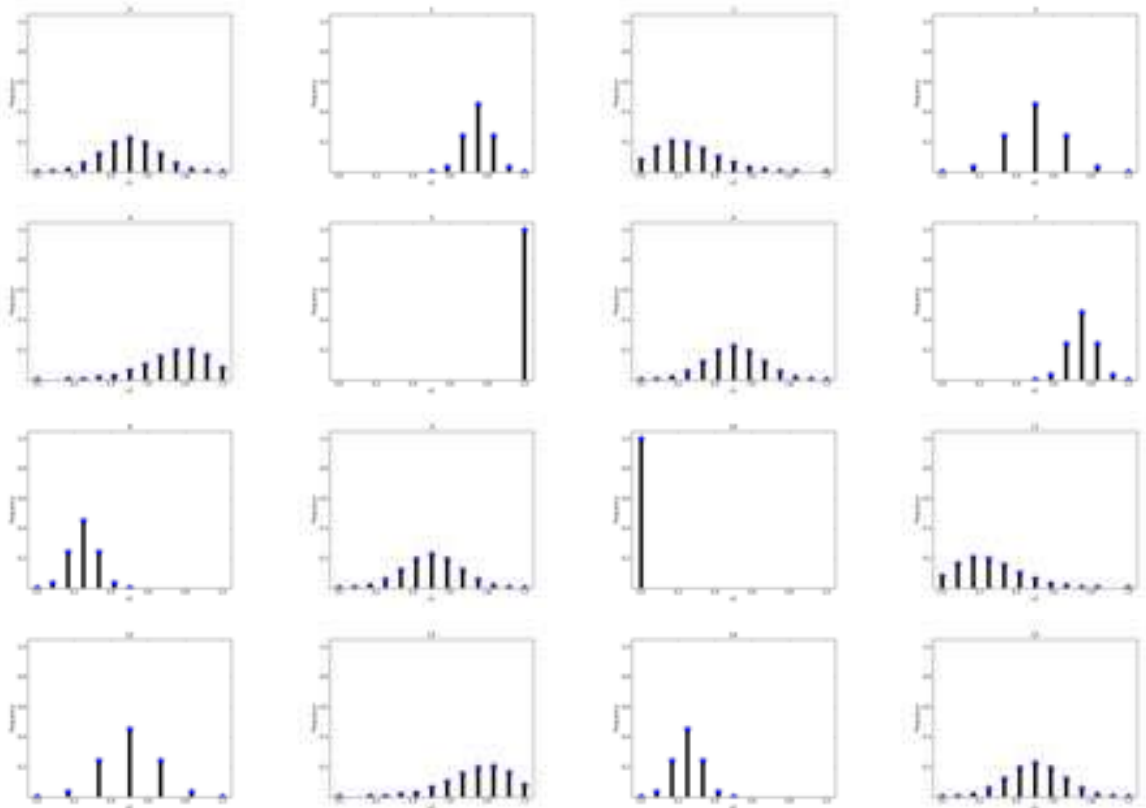

(M4) $u_{1}$ SL group 

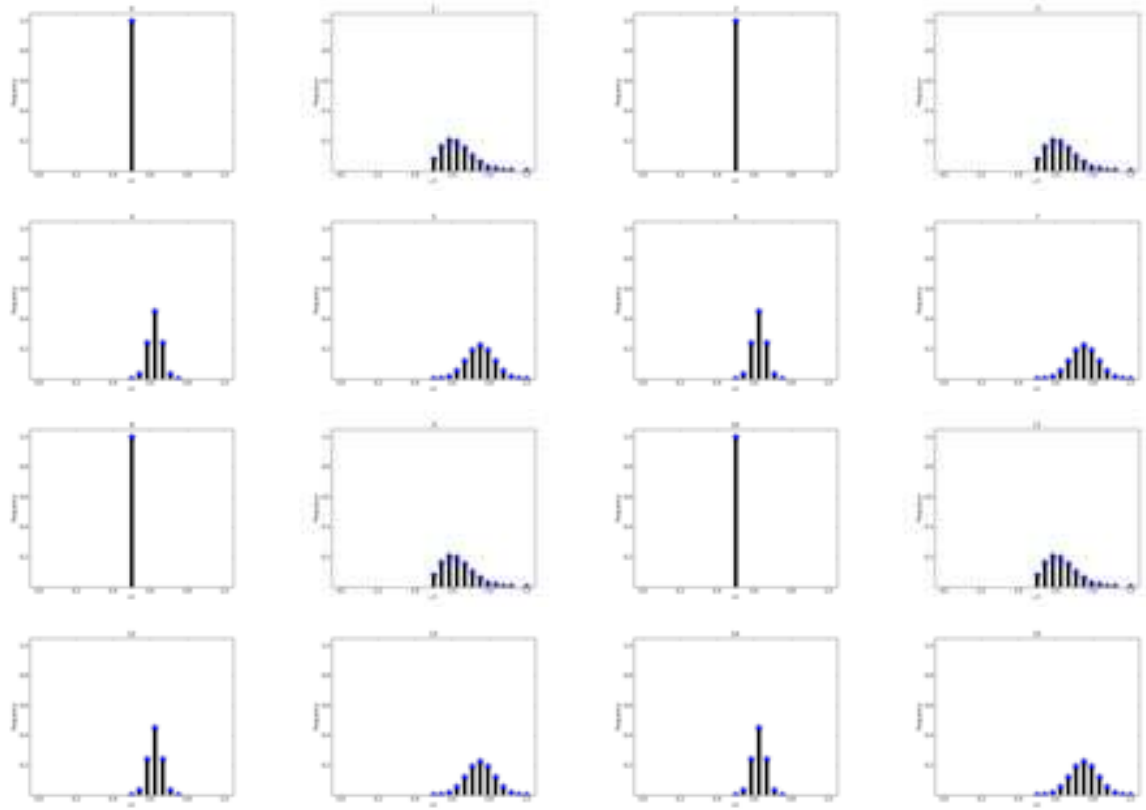

(M5) $v_{+}$SL group
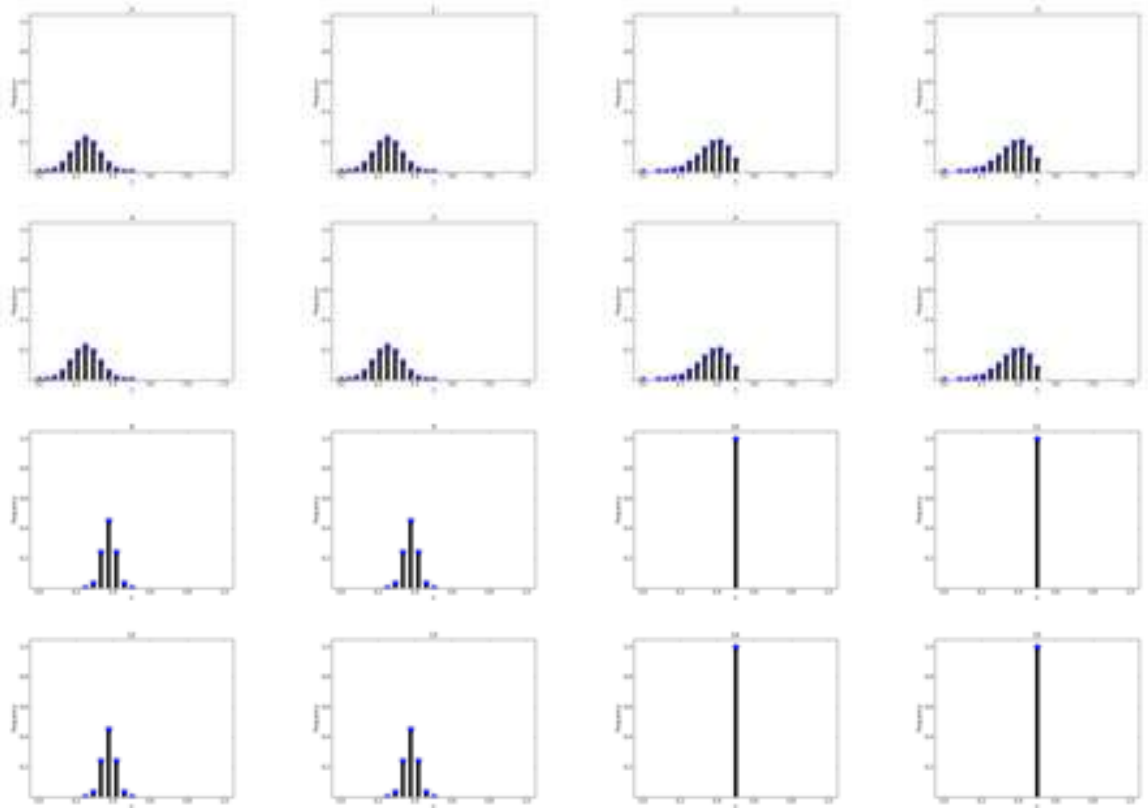

(M6) $v_{-}$SL group 

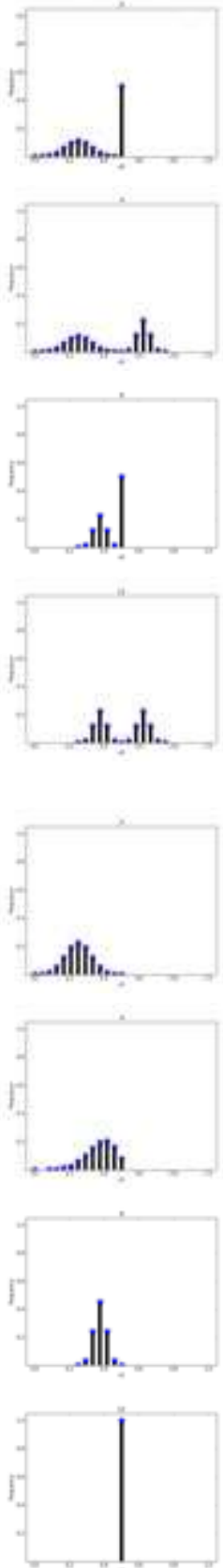
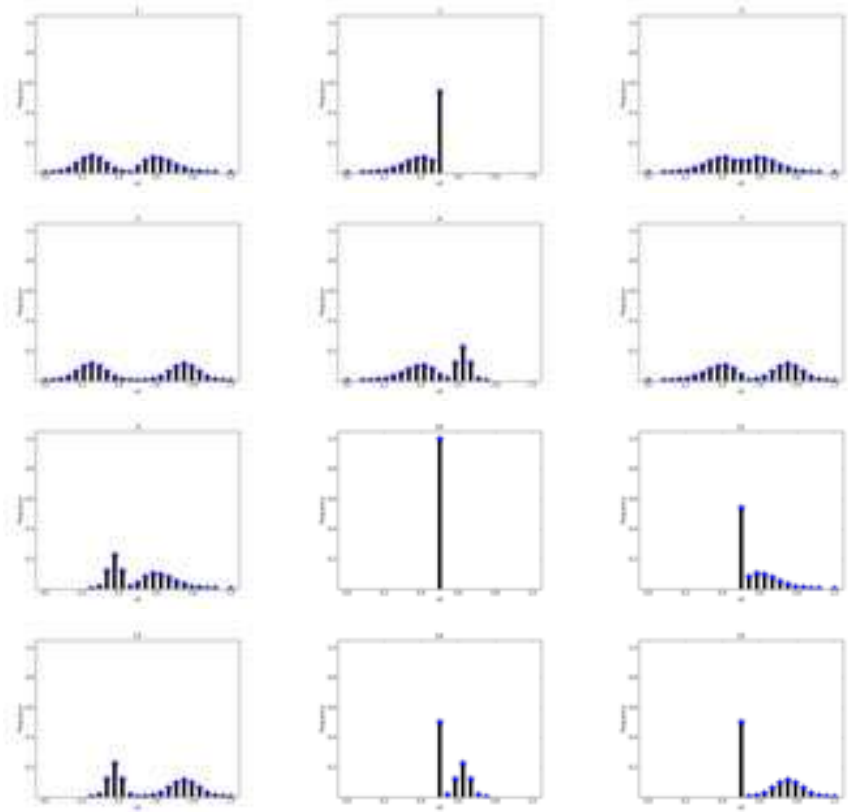

(M7) $v_{0}$ SL group
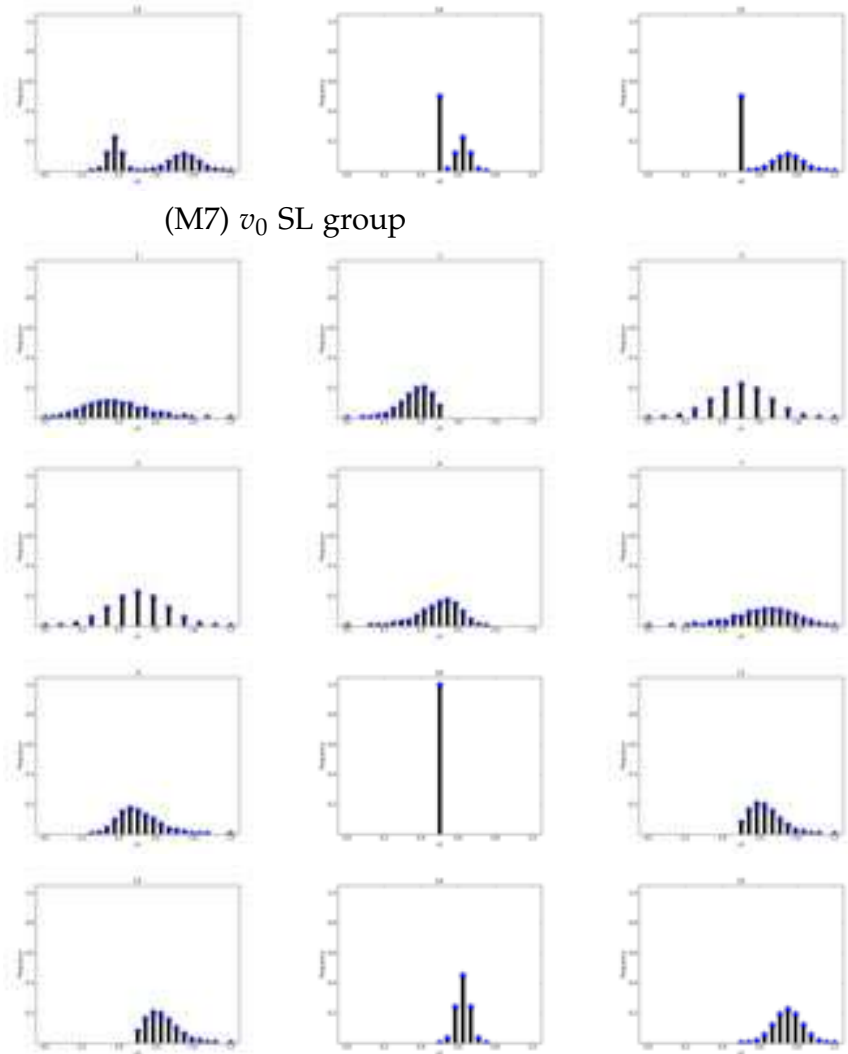

(M8) $v_{1}$ SL group 

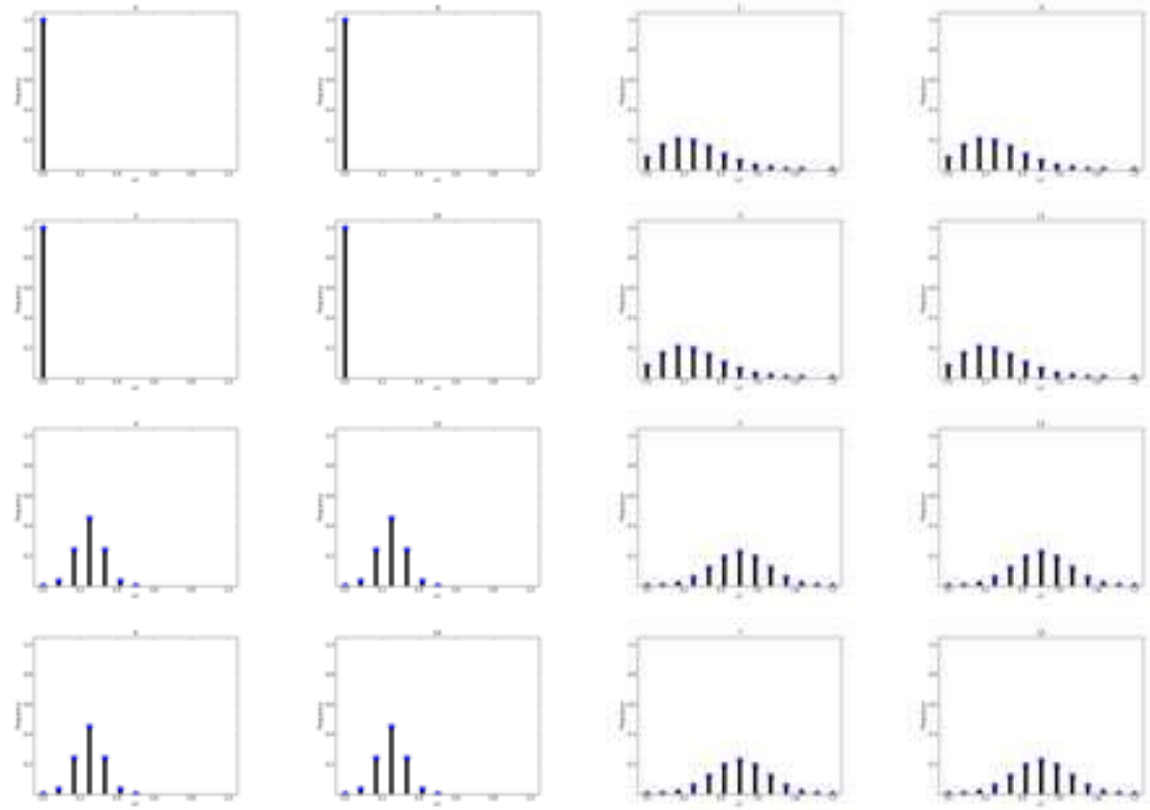

(M9) $u_{+} \mathrm{W}$ group
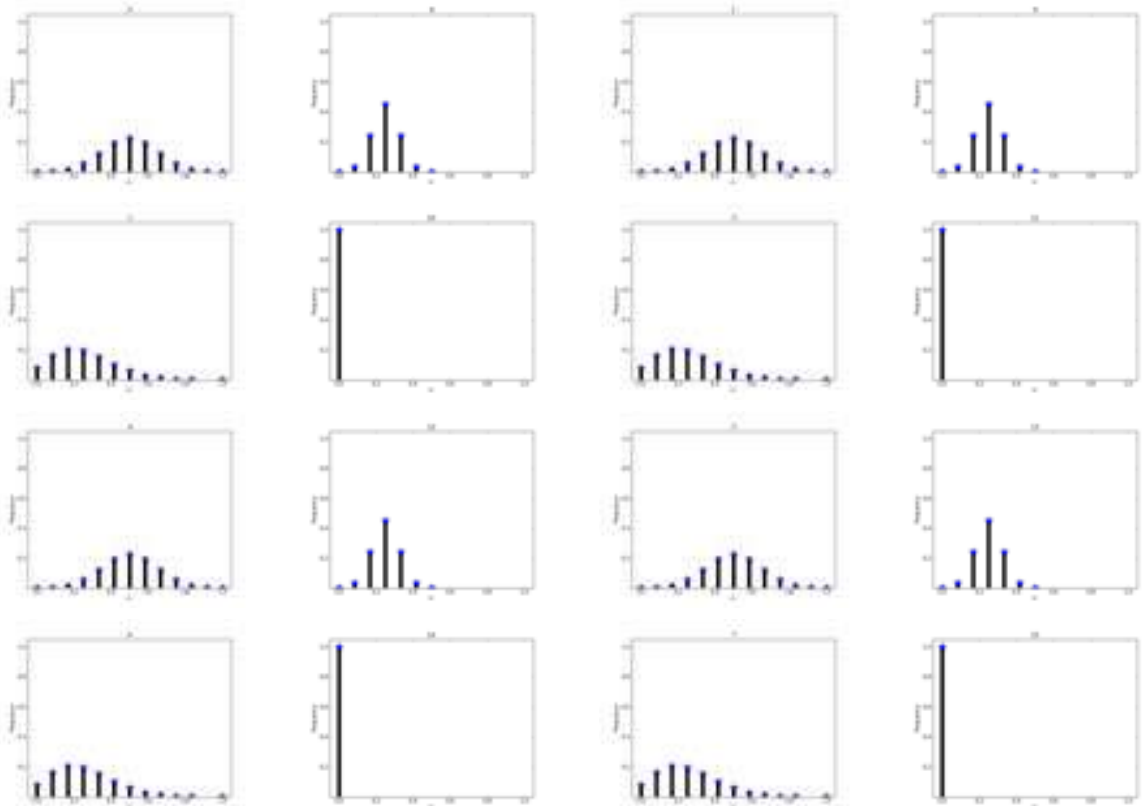

(M10) $u_{-}$W group 

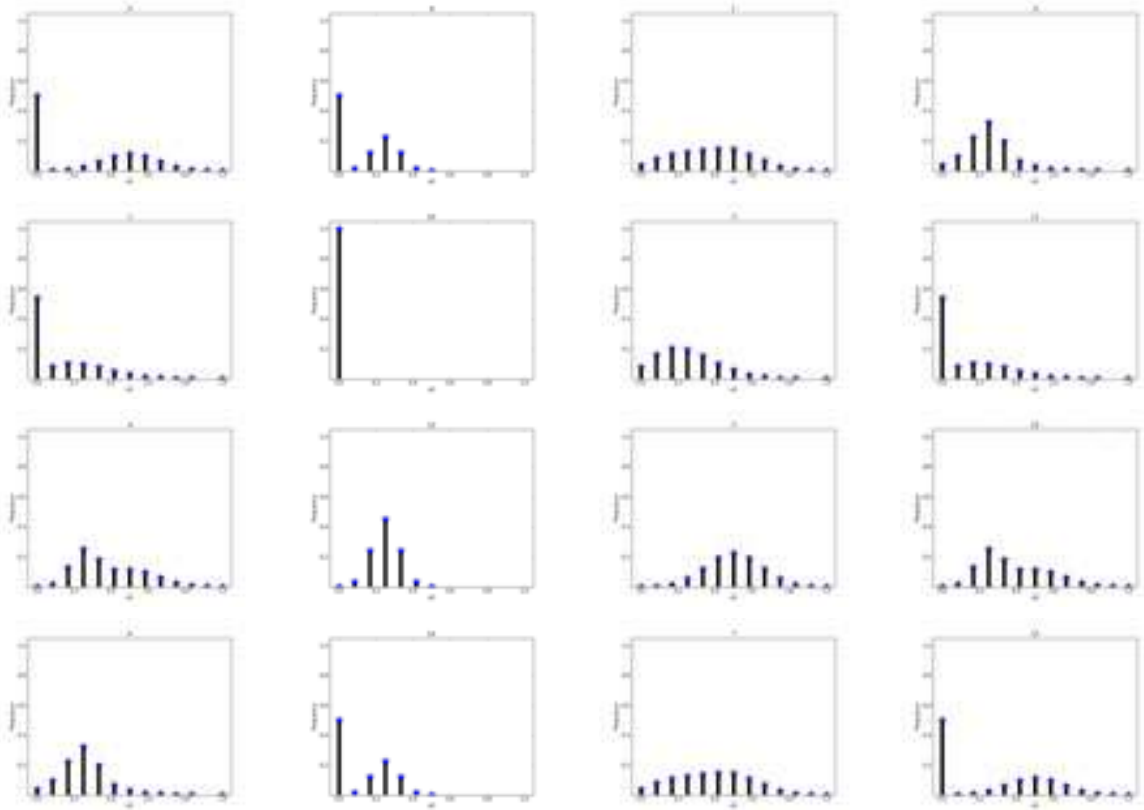

(M11) $u_{0} \mathrm{~W}$ group
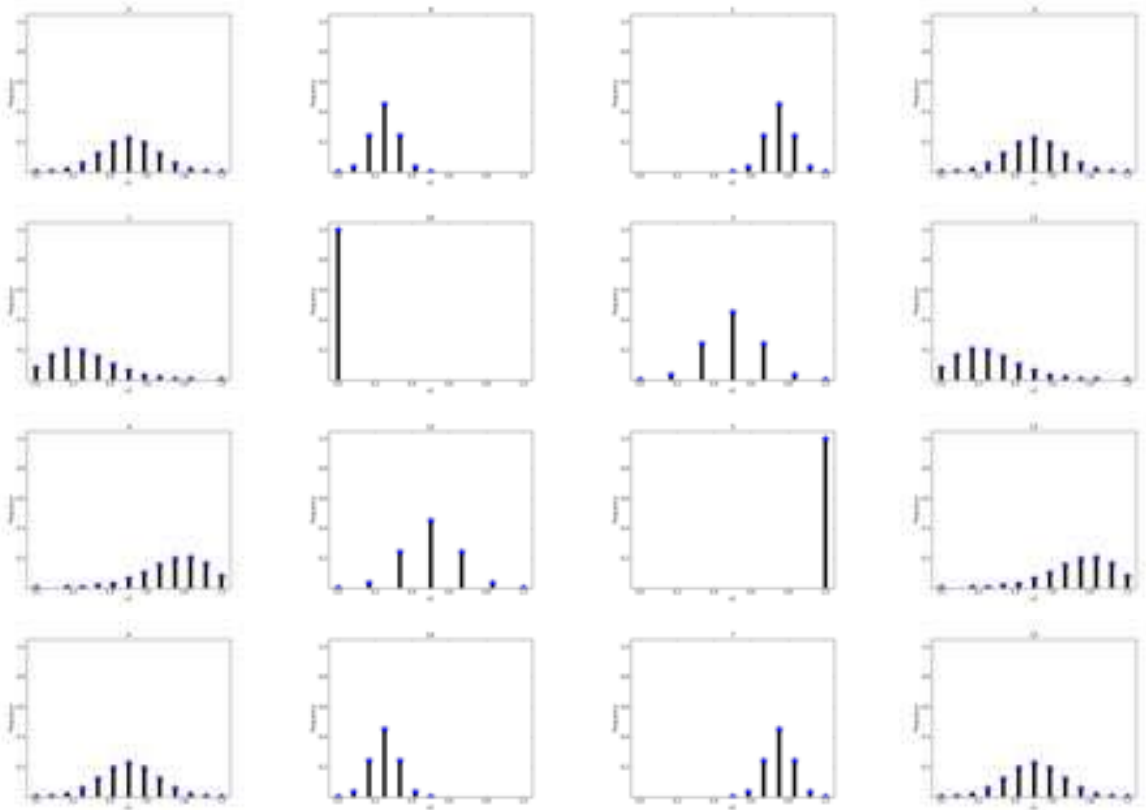

(M12) $u_{1} \mathrm{~W}$ group 

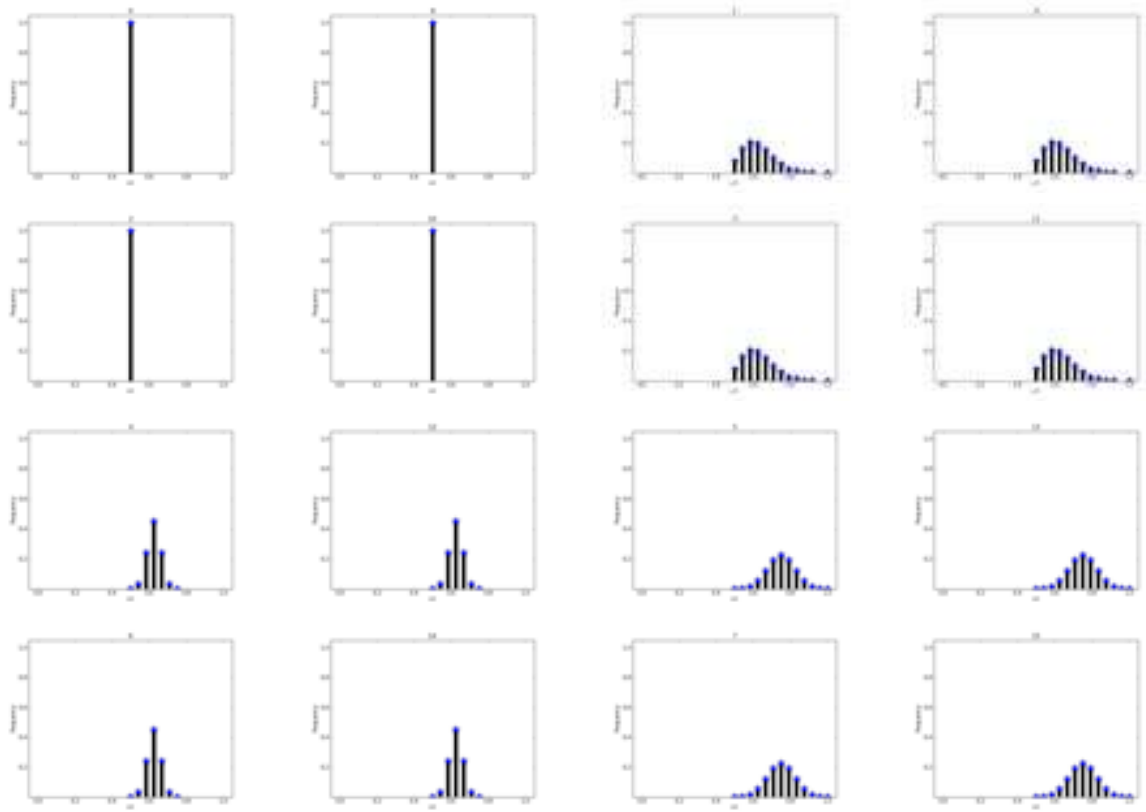

(M13) $v_{+} \mathrm{W}$ group
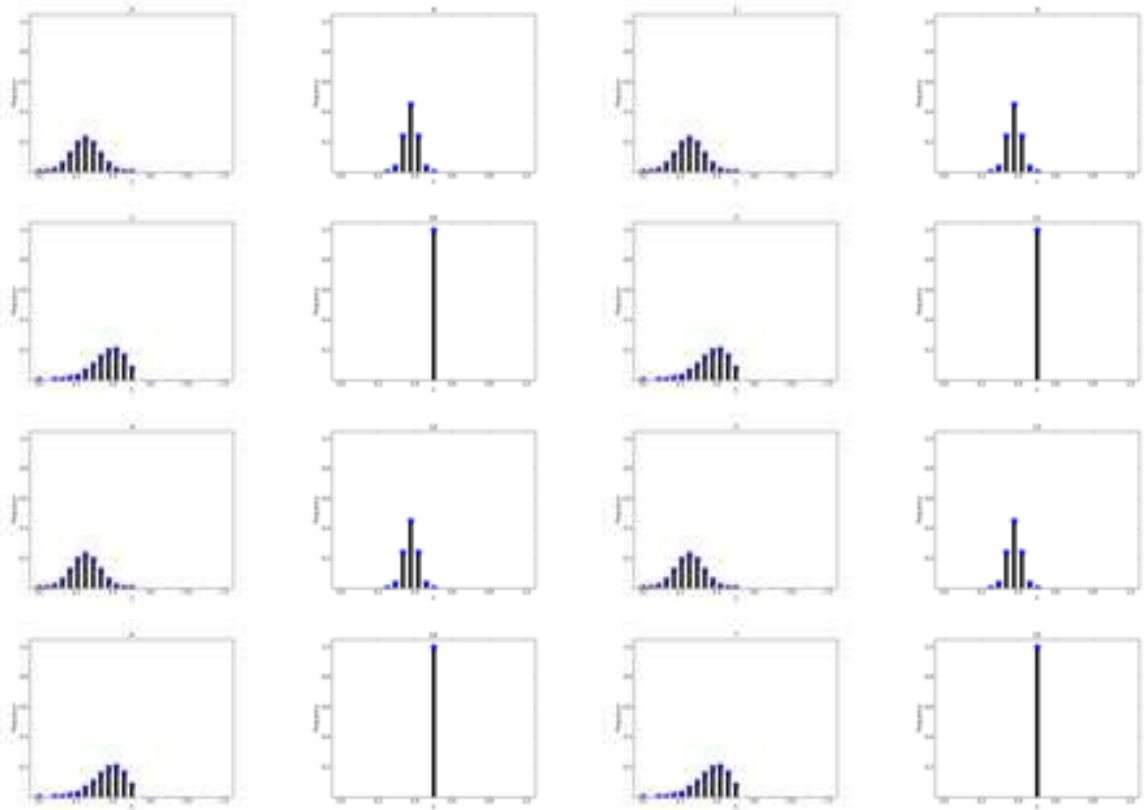

(M14) $v_{-}$W group 

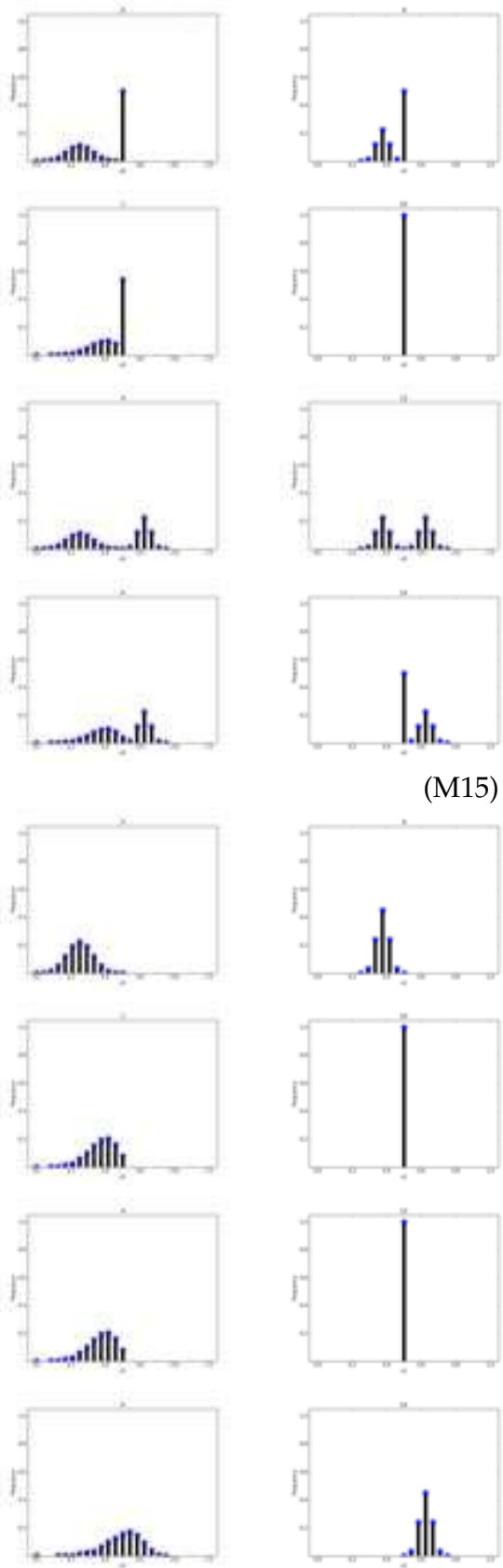
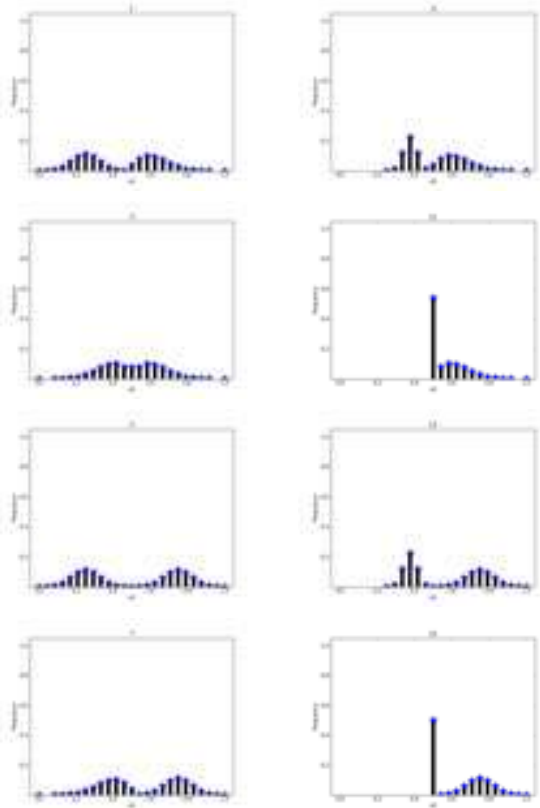

(M15) $v_{0} \mathrm{~W}$ group
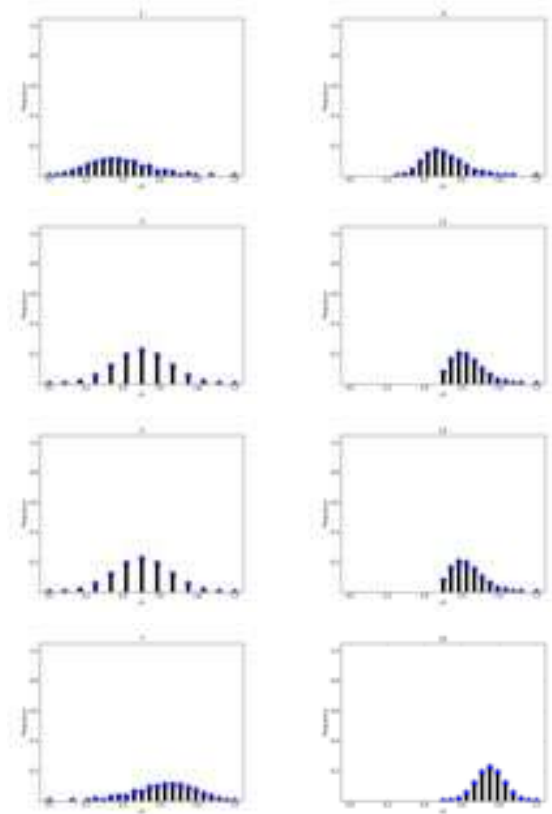

(M16) $v_{1} \mathrm{~W}$ group 

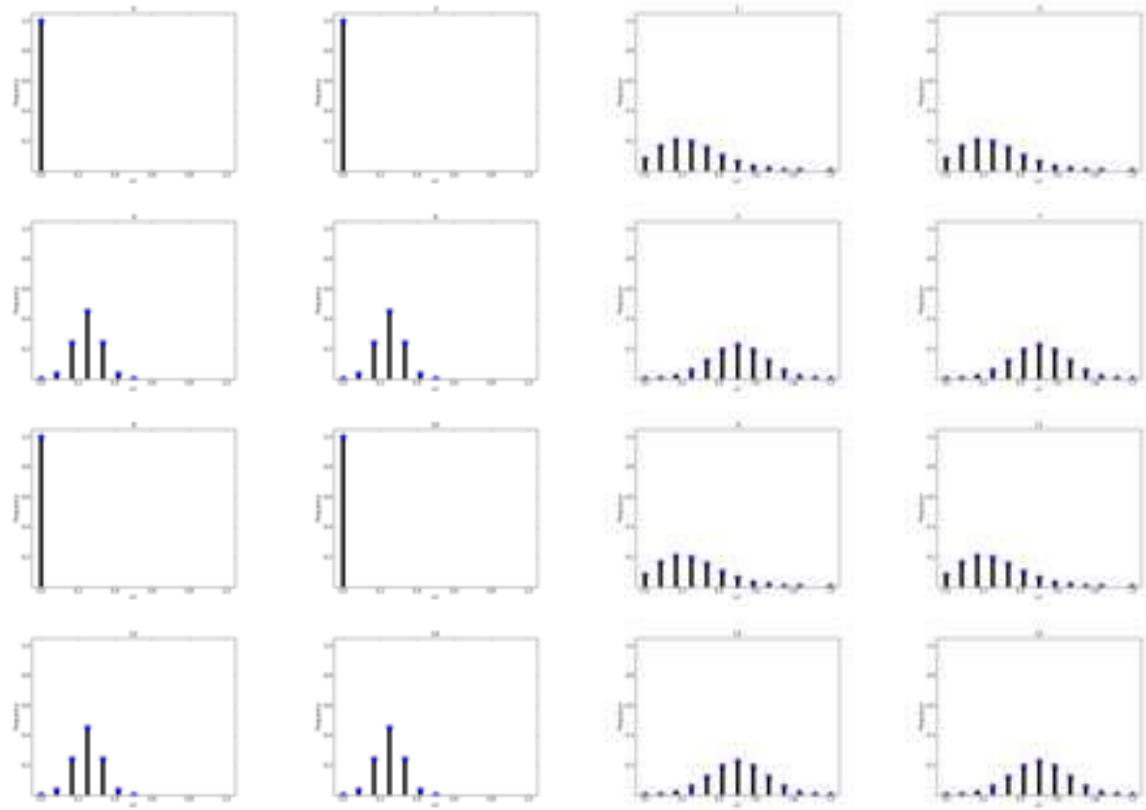

(M17) $u_{+}$F group
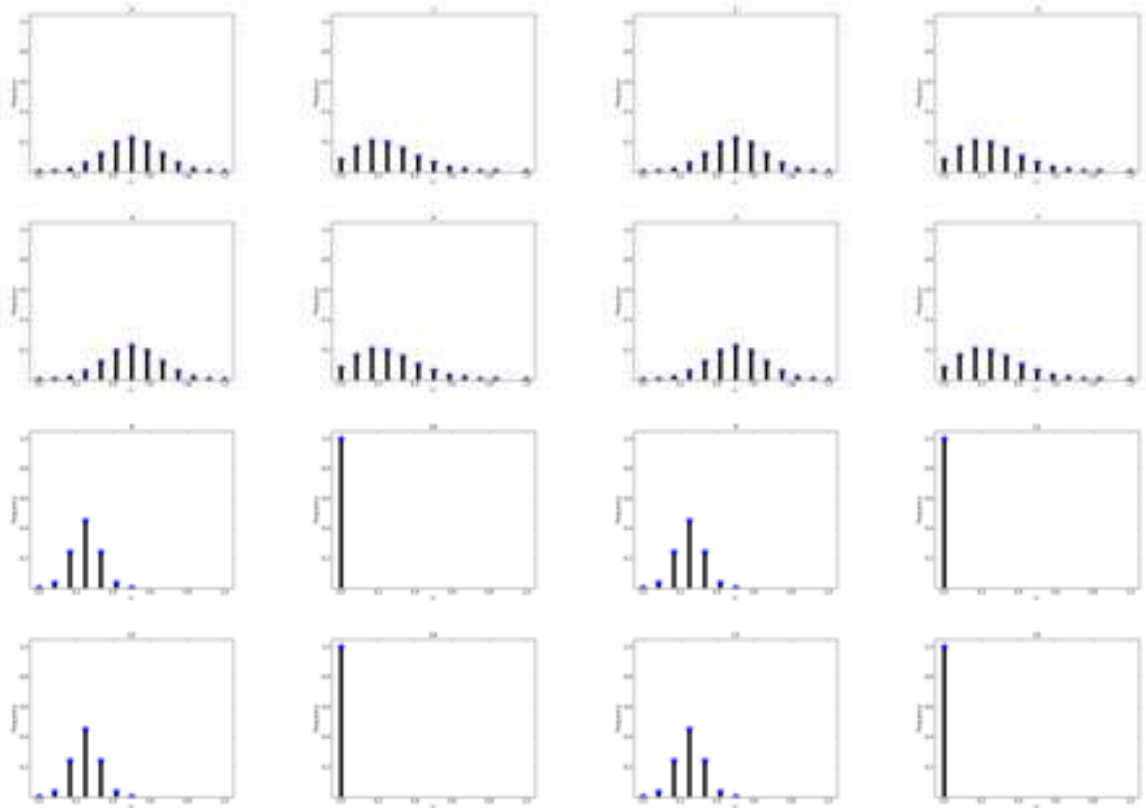

(M18) $u_{-}$F group 

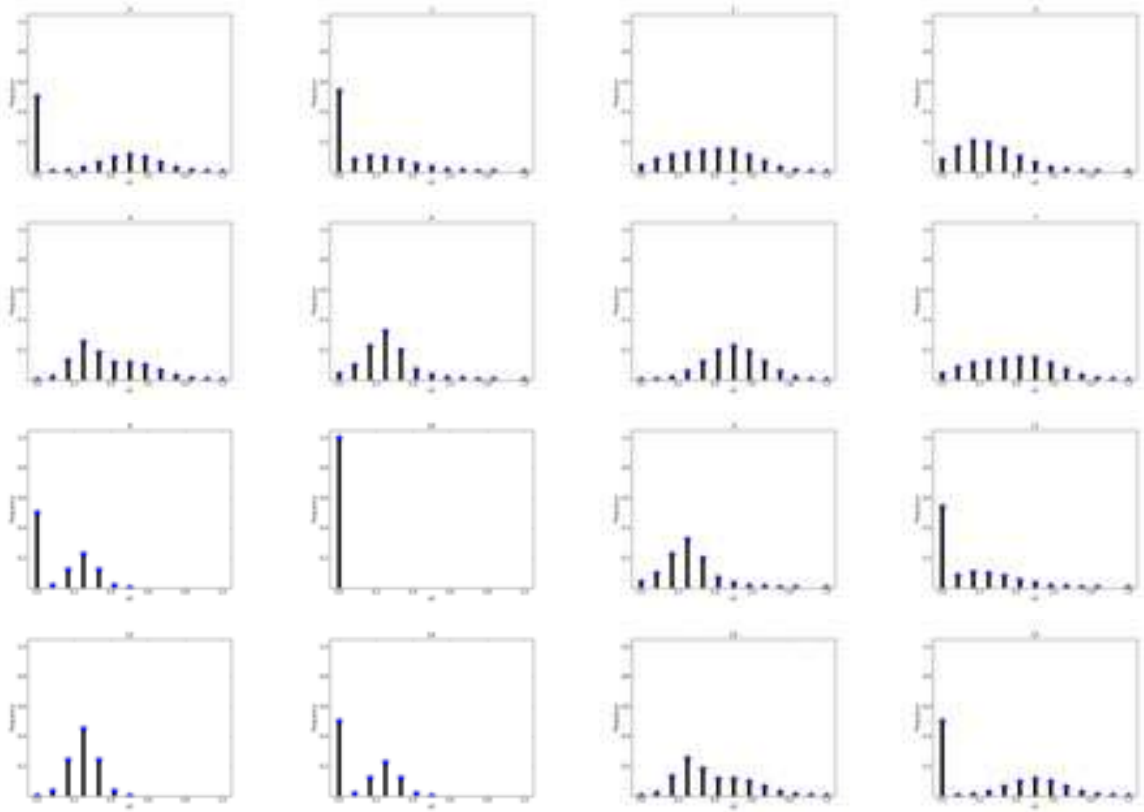

(M19) $u_{0}$ F group
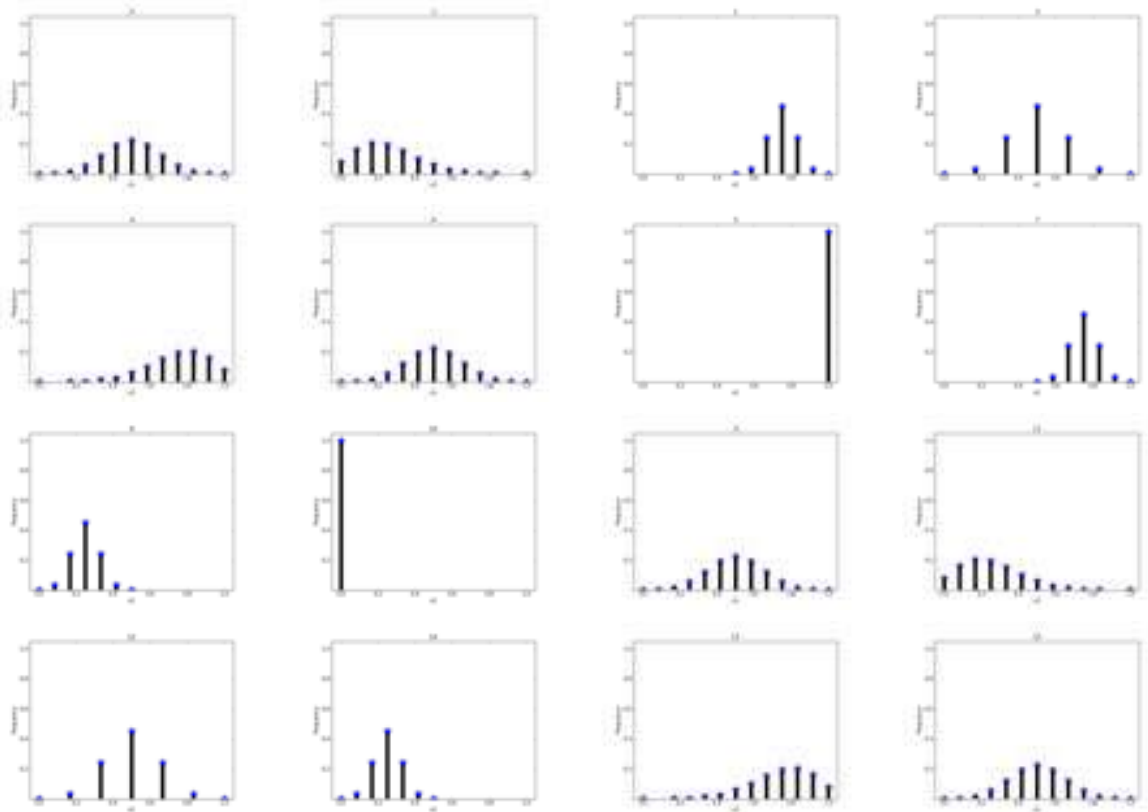

(M20) $u_{1}$ F group 

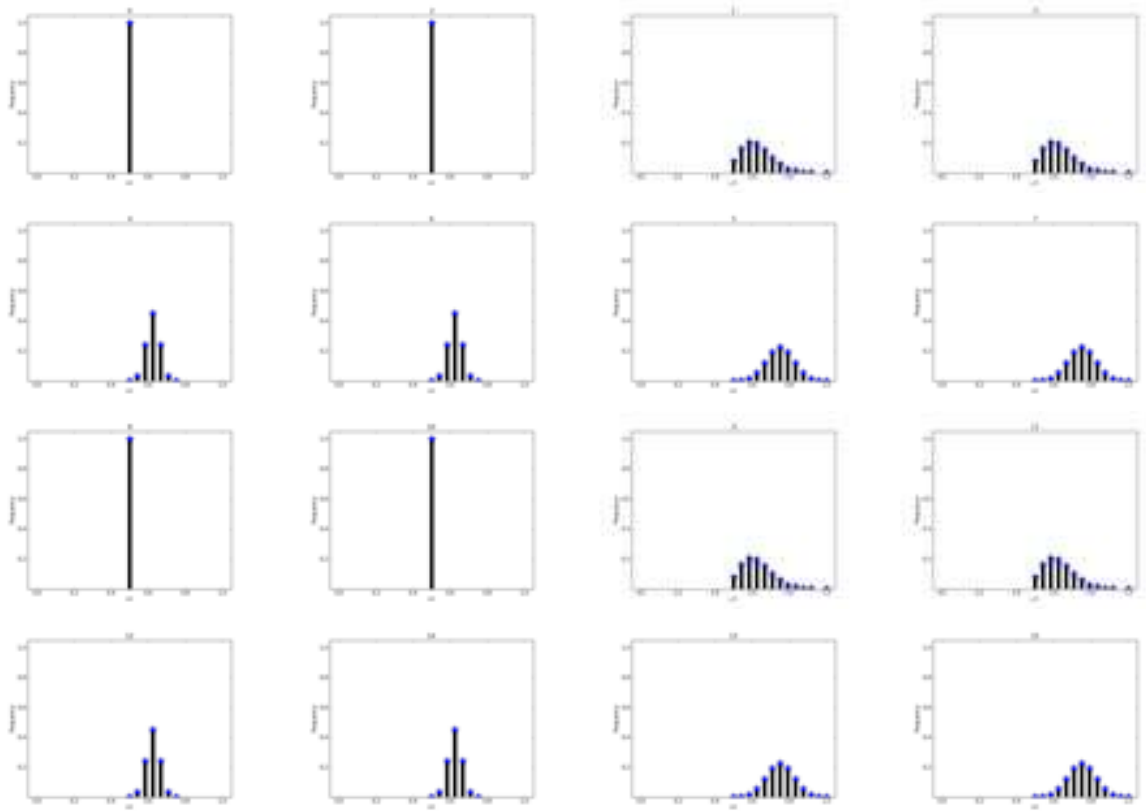

$(\mathrm{M} 21) v_{+} \mathrm{F}$ group
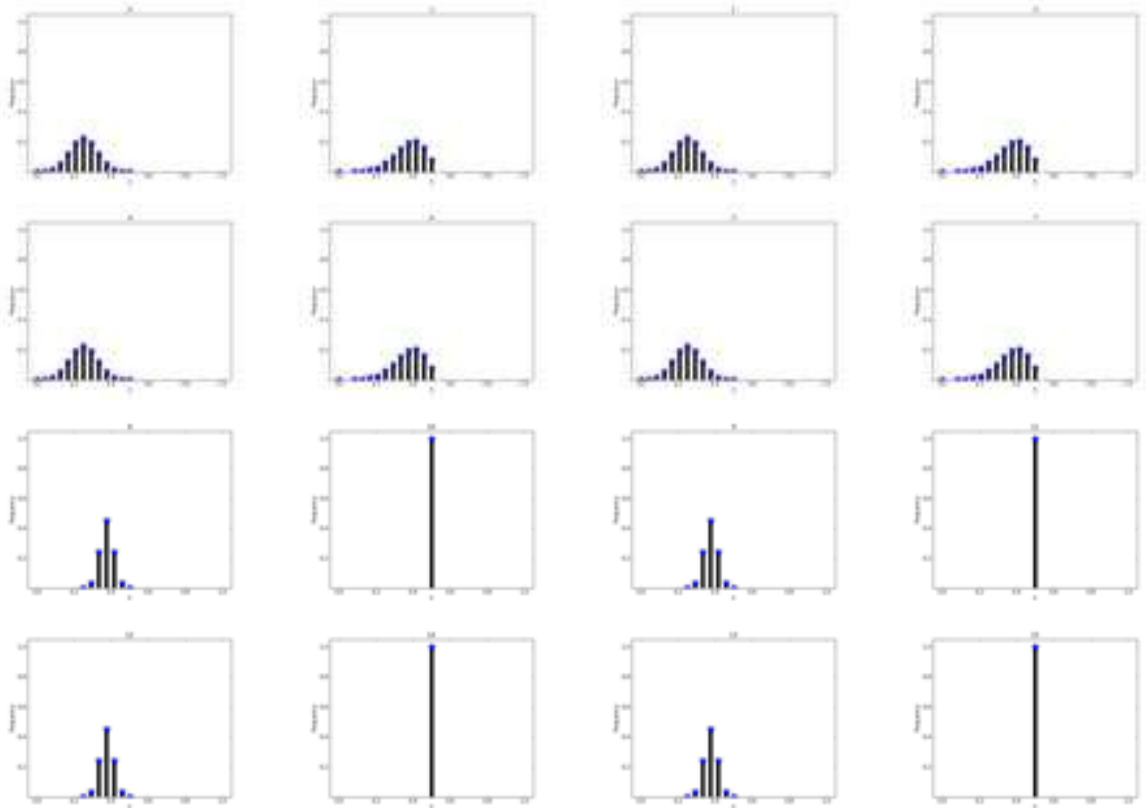

(M22) $v_{-}$F group 

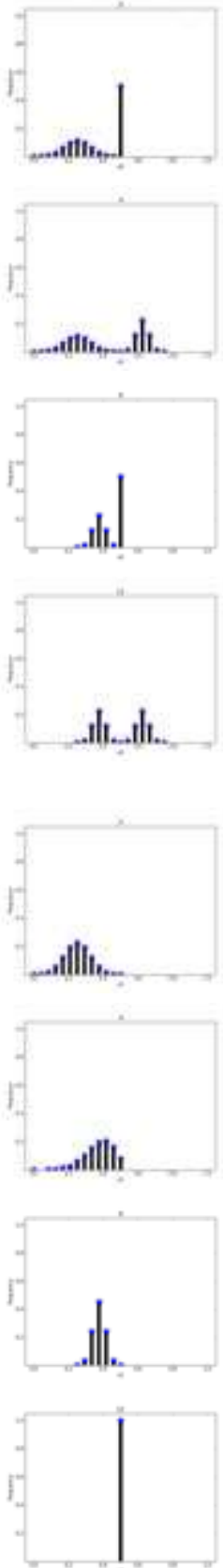
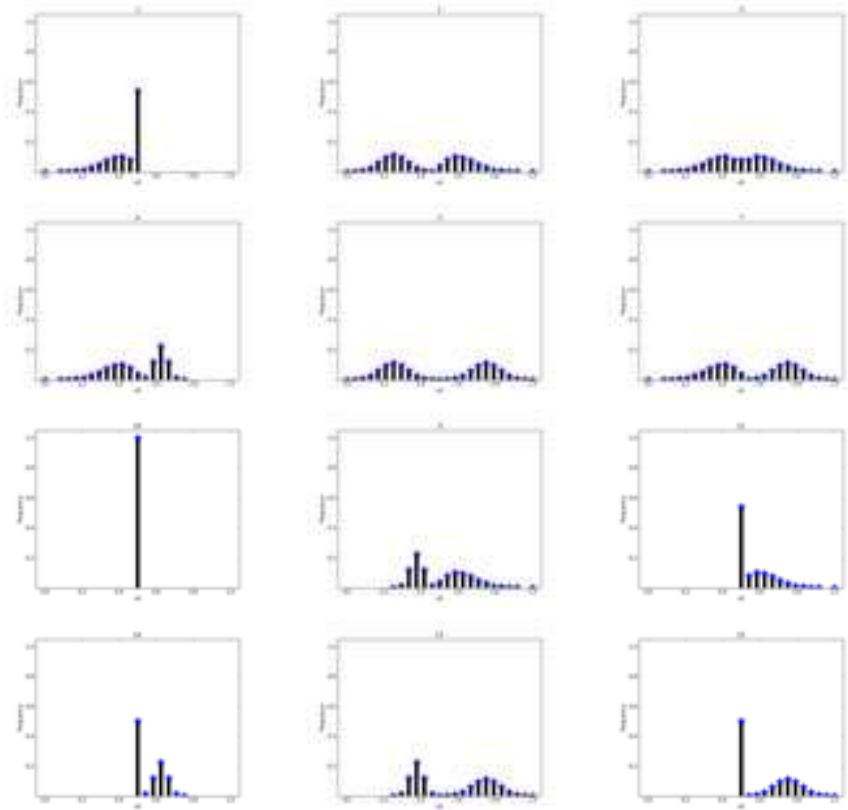

(M23) $v_{0} \mathrm{~F}$ group
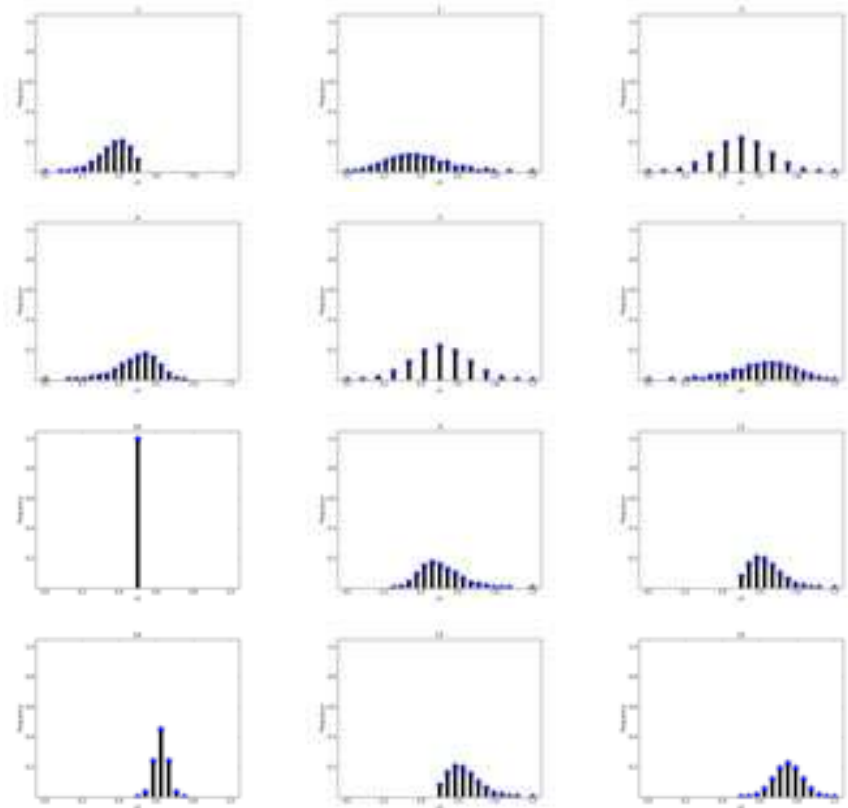

(M24) $v_{1} \mathrm{~F}$ group 

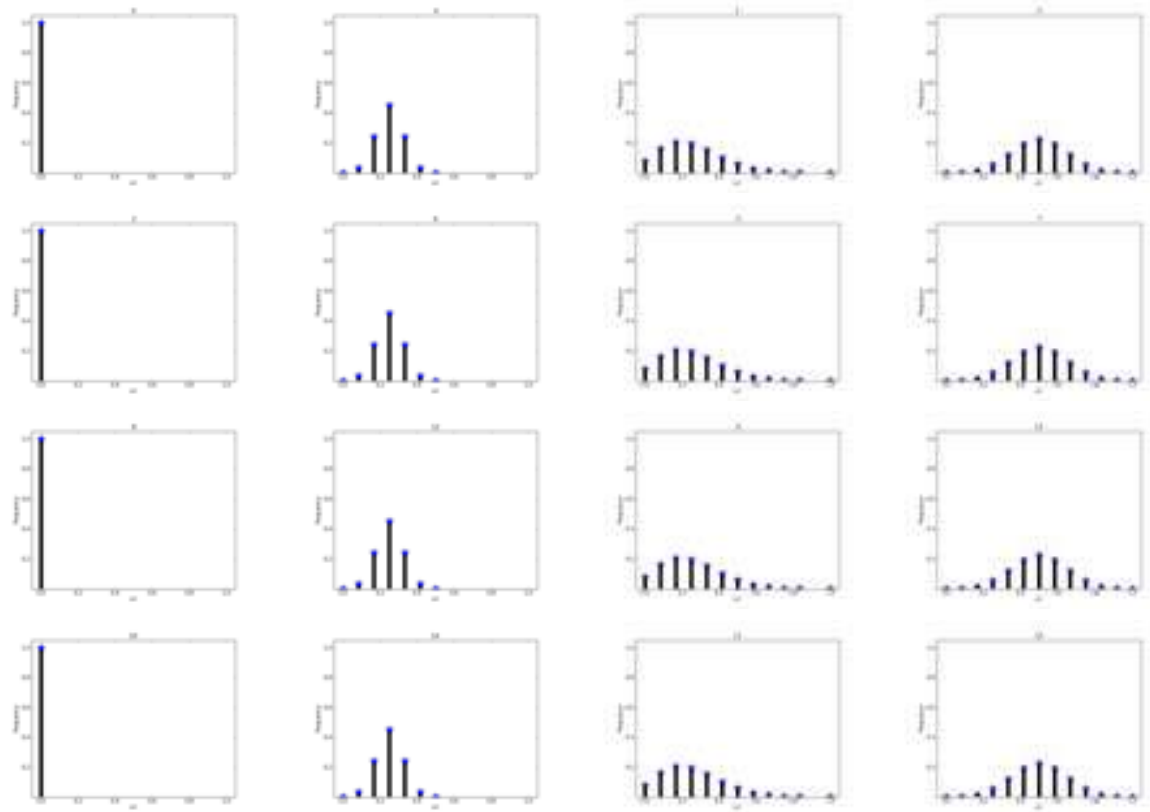

(M25) $u_{+}$C group
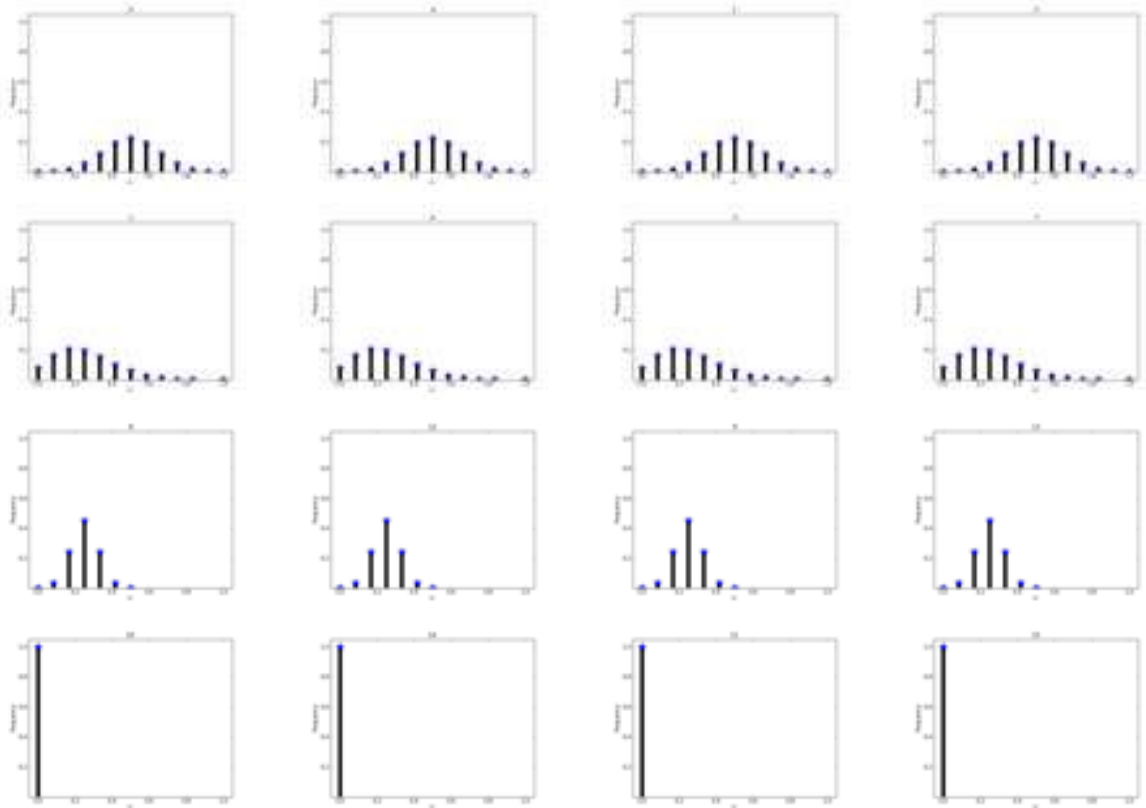

(M26) $u_{-}$C group 

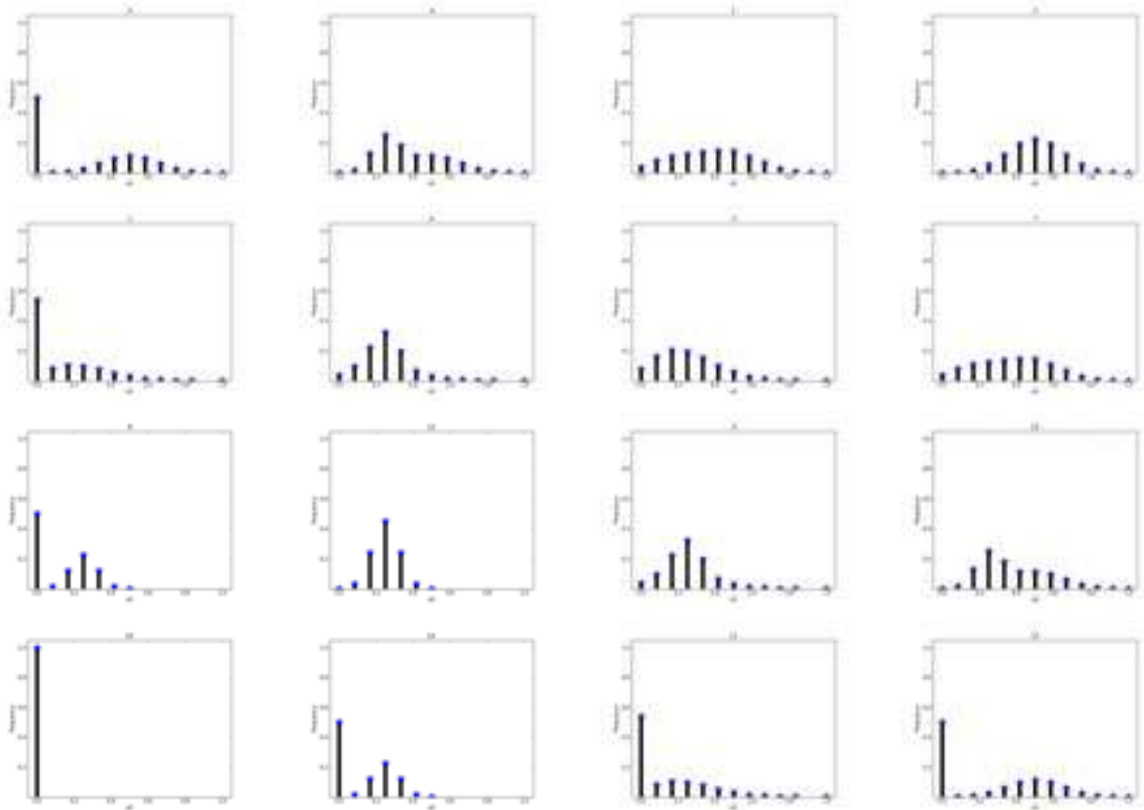

(M27) $u_{0}$ C group
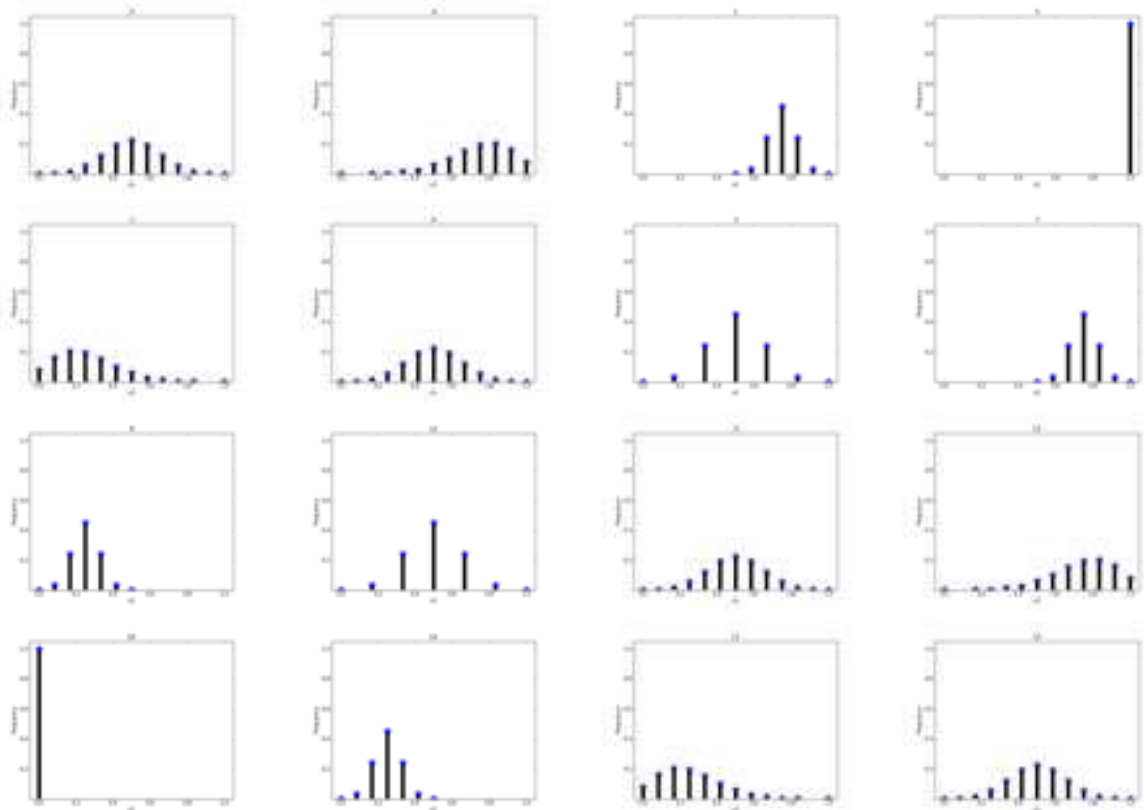

(M28) $u_{1}$ C group 

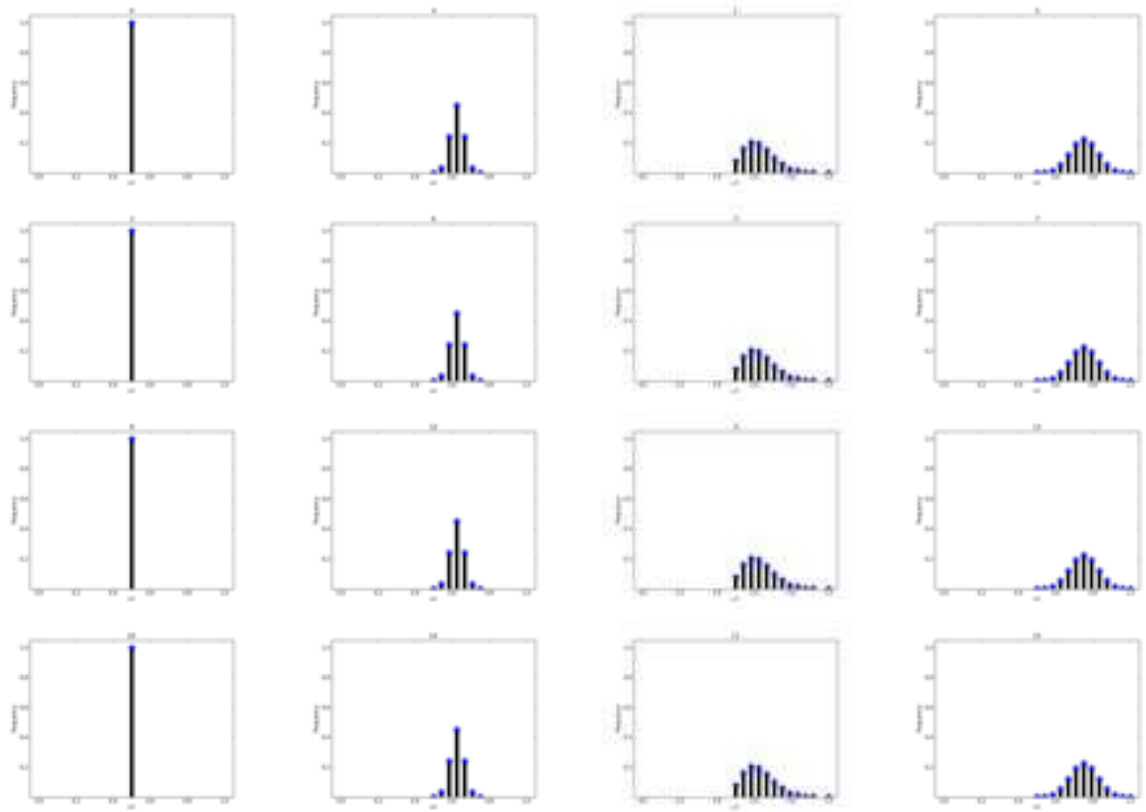

(M29) $v_{+}$C group
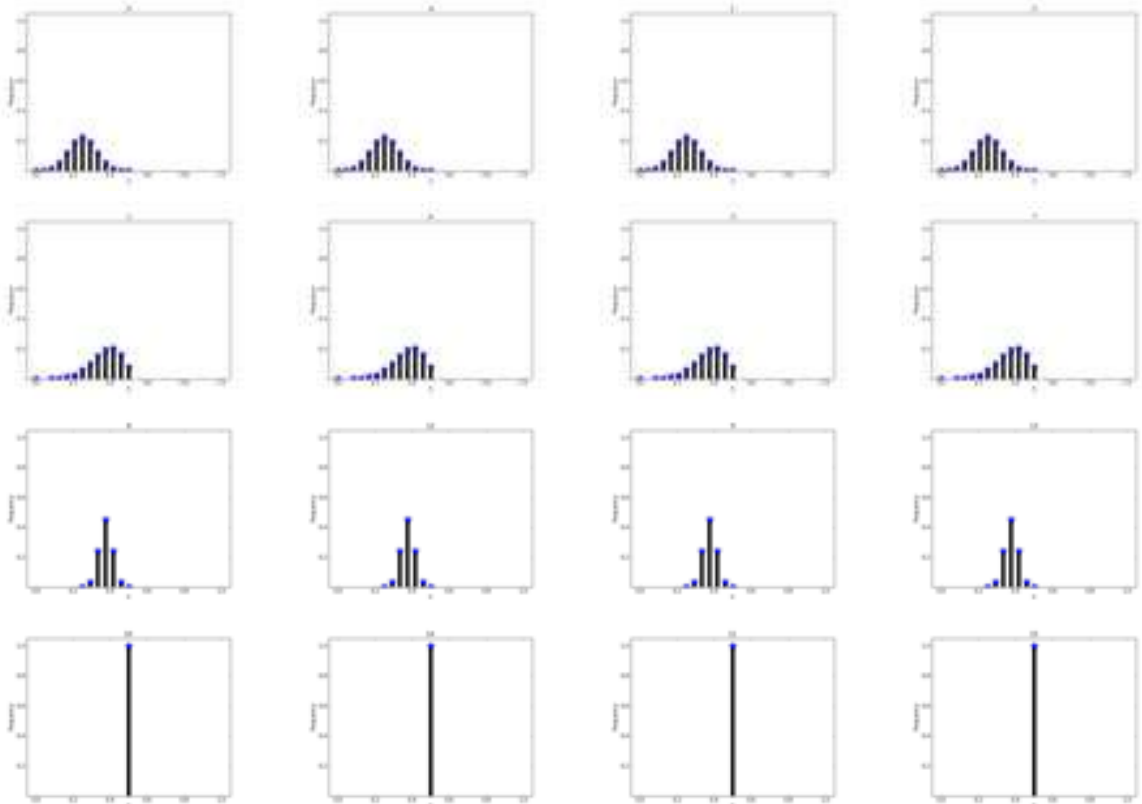

(M30) $v_{-}$C group 

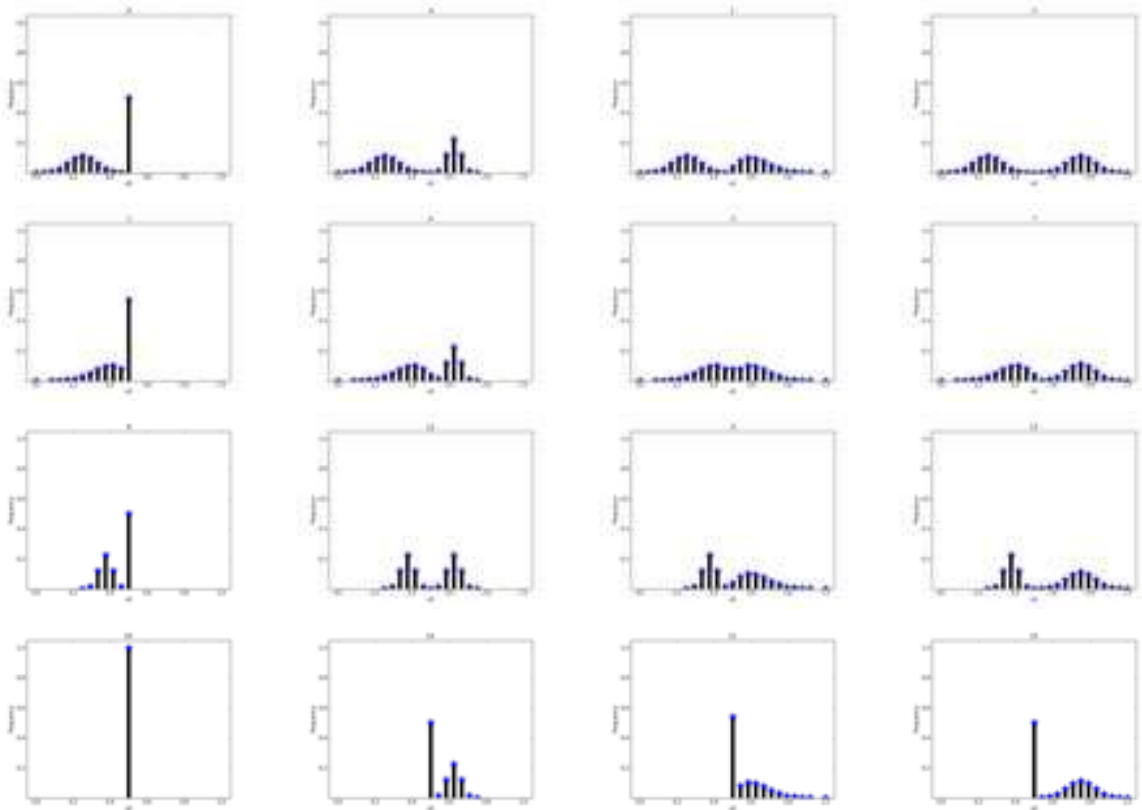

$(\mathrm{M} 31) v_{0} \mathrm{C}$ group
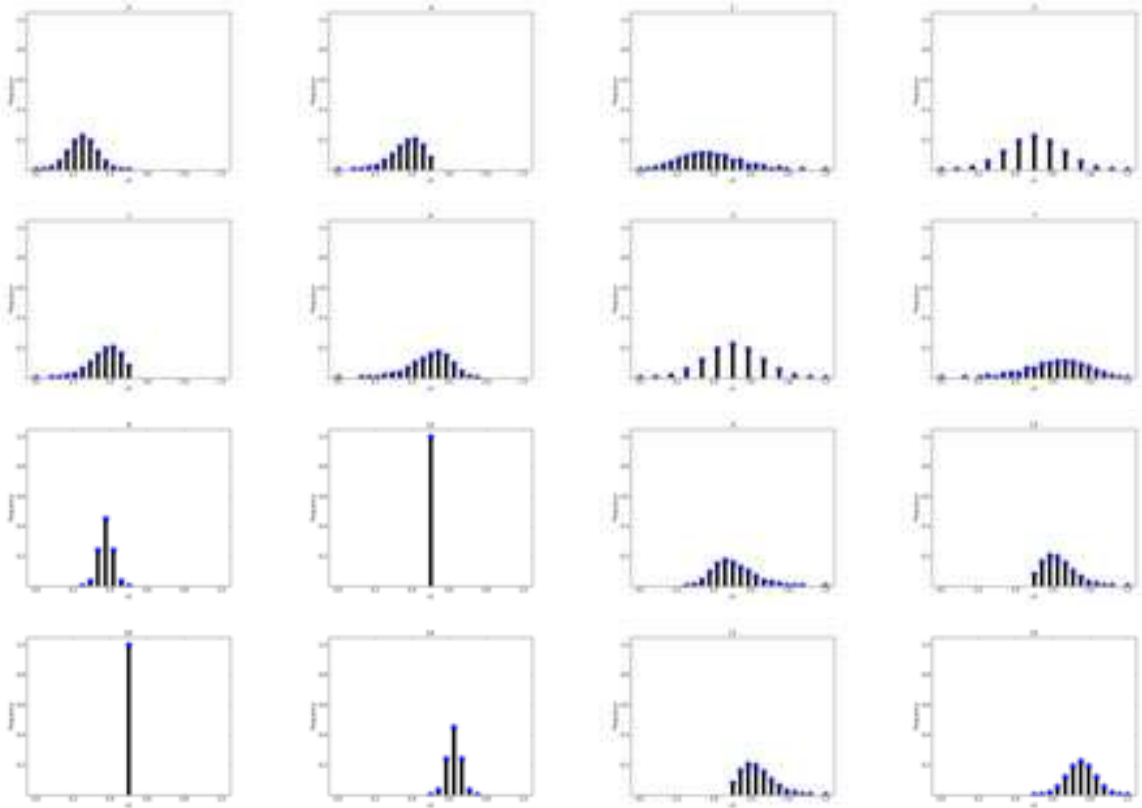

(M32) $v_{1} \mathrm{C}$ group

Figure 9. (M1-M32) IMM for MPS; (M1-M8) SL group; (M9-M16) W group; (M17-M24) F group; (M25-M-32) C group. 

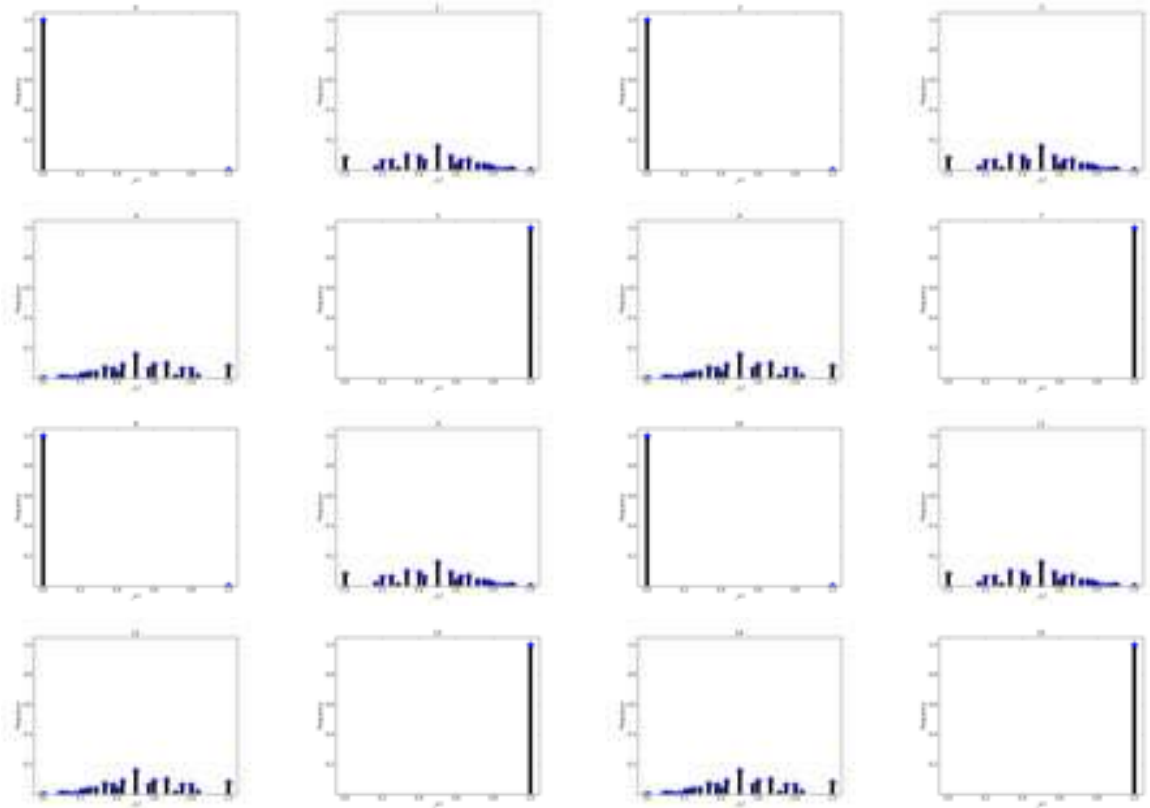

(C1) $\tilde{u}_{+}$SL group
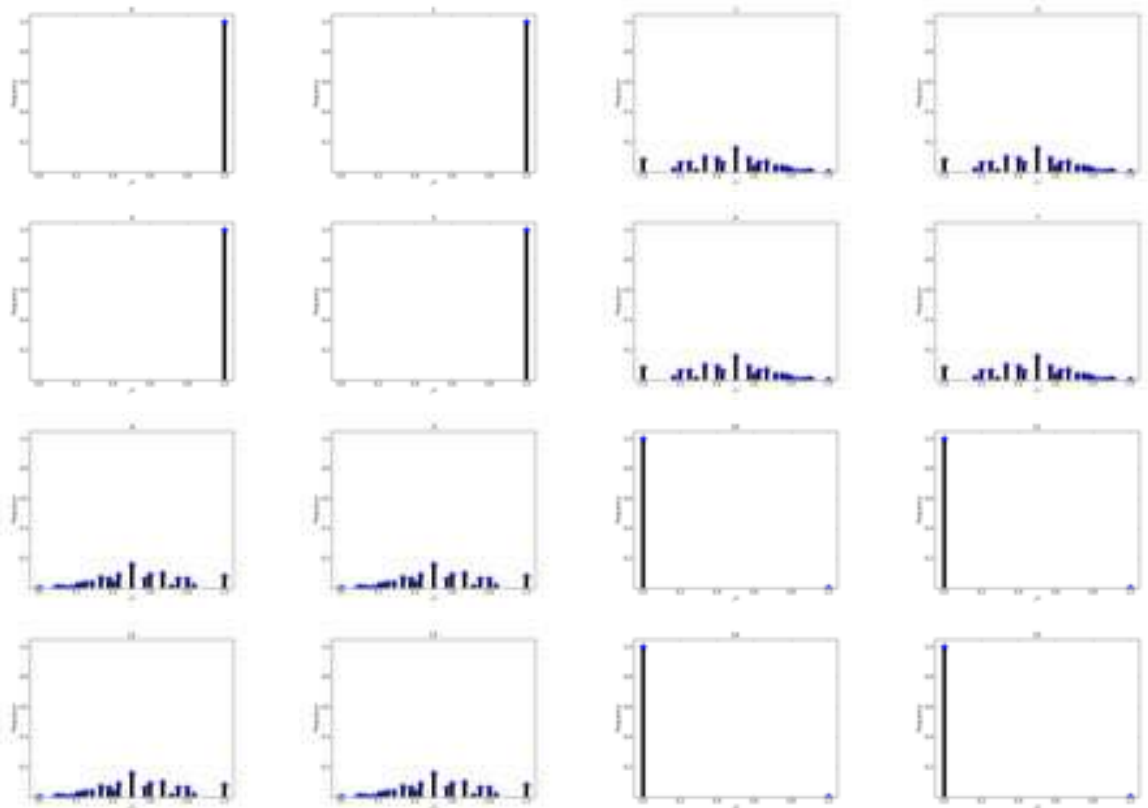

(C2) $\tilde{u}_{-}$SL group 

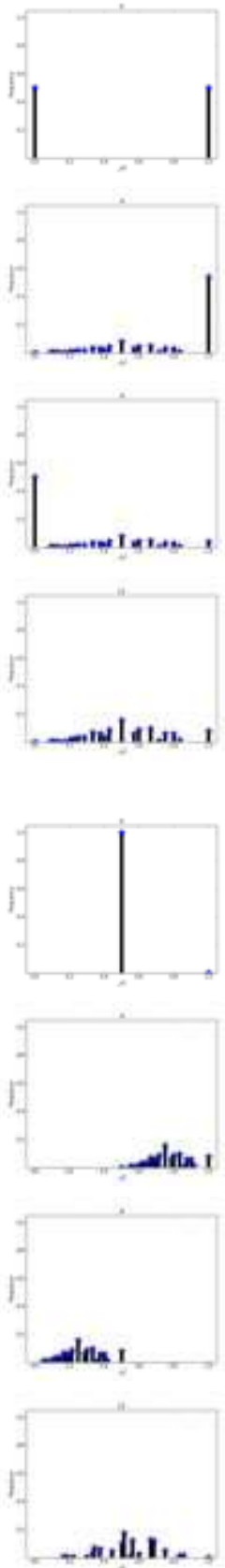
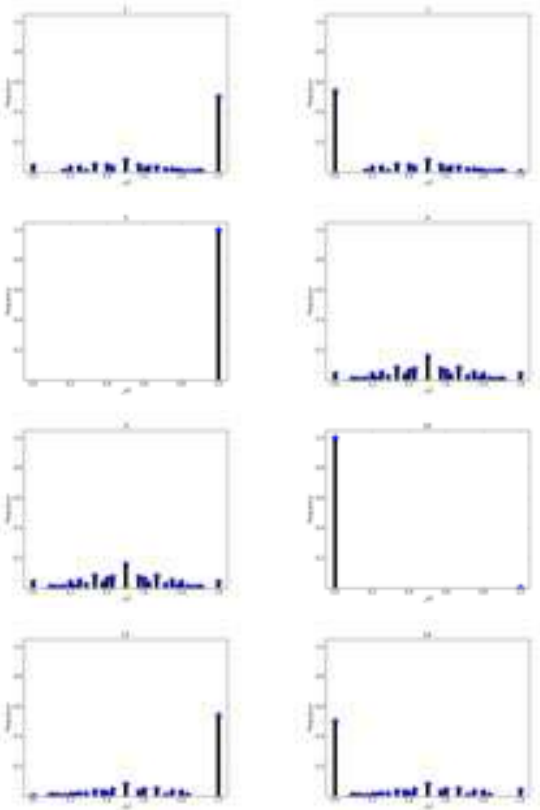

(C3) $\tilde{u}_{0}$ SL group
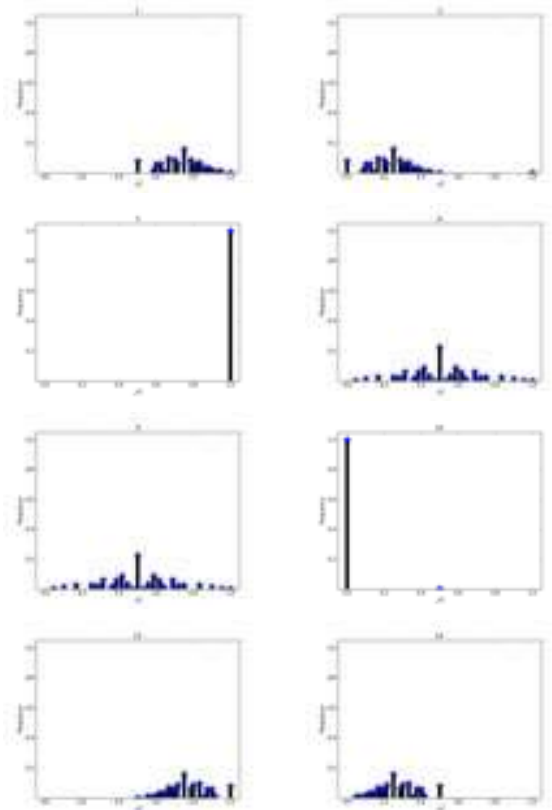
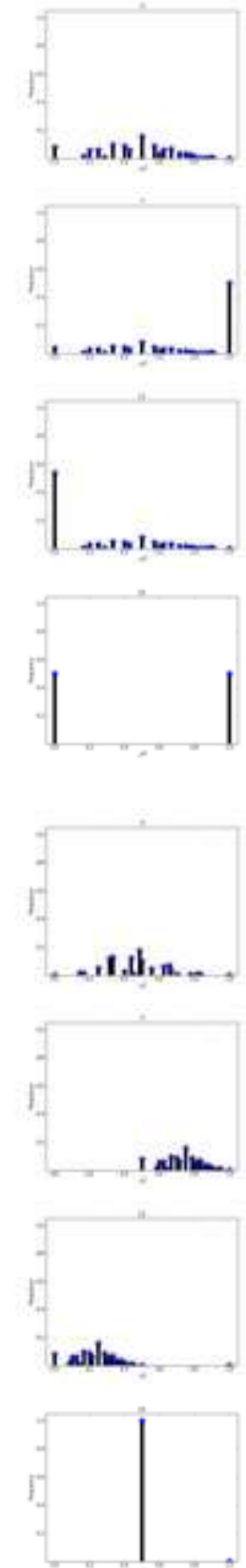

(C4) $\tilde{u}_{1}$ SL group 
Interactive Maps on Variant Phase Spaces

179

http://dx.doi.org/10.5772/51635
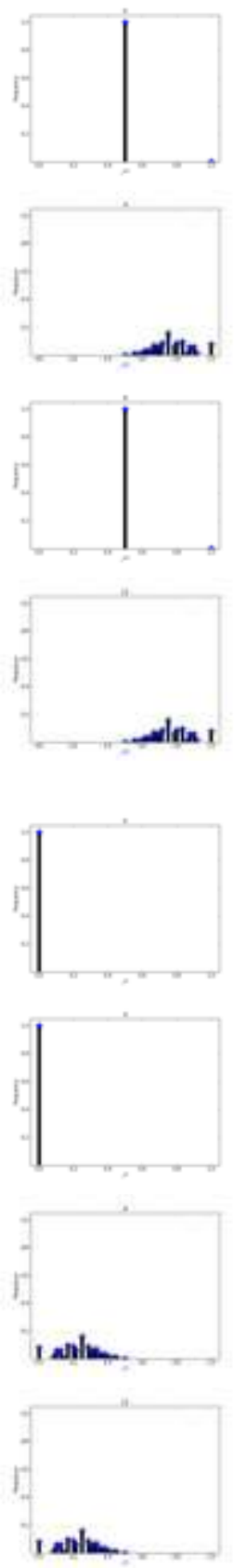
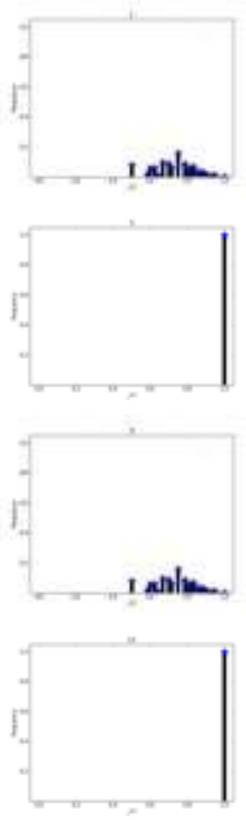

(C5) $\tilde{v}_{+}$SL group
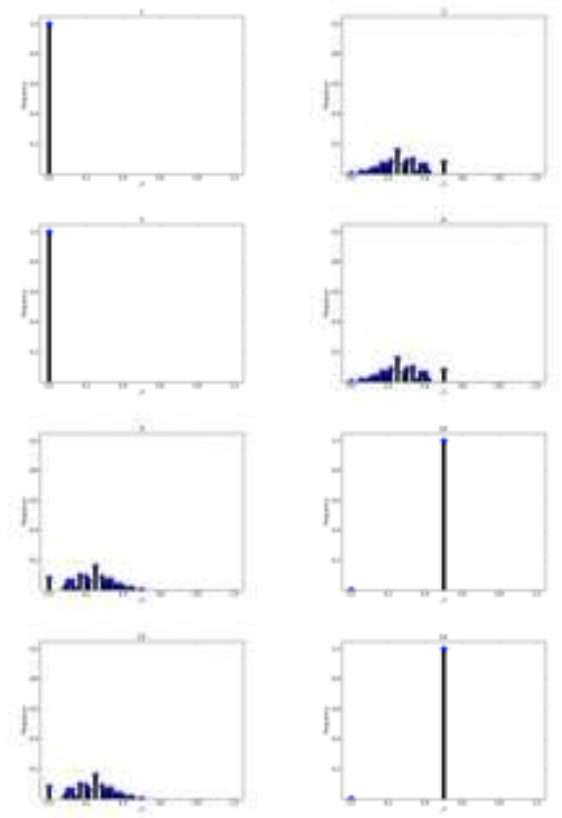

(C6) $\tilde{v}_{-}$SL group
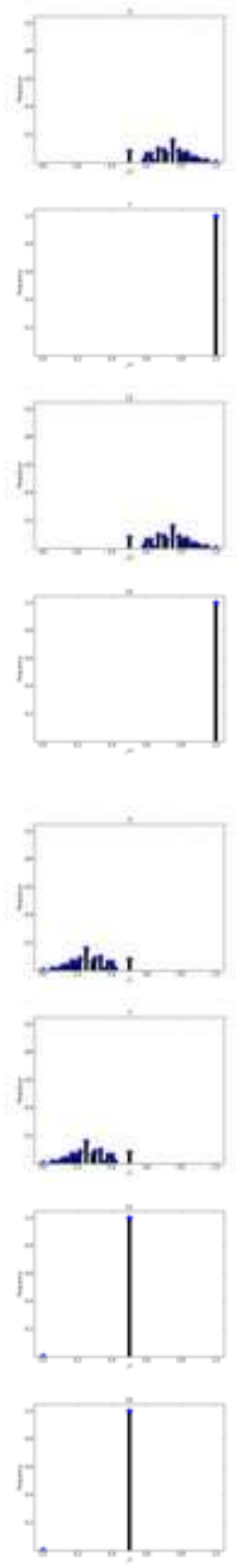

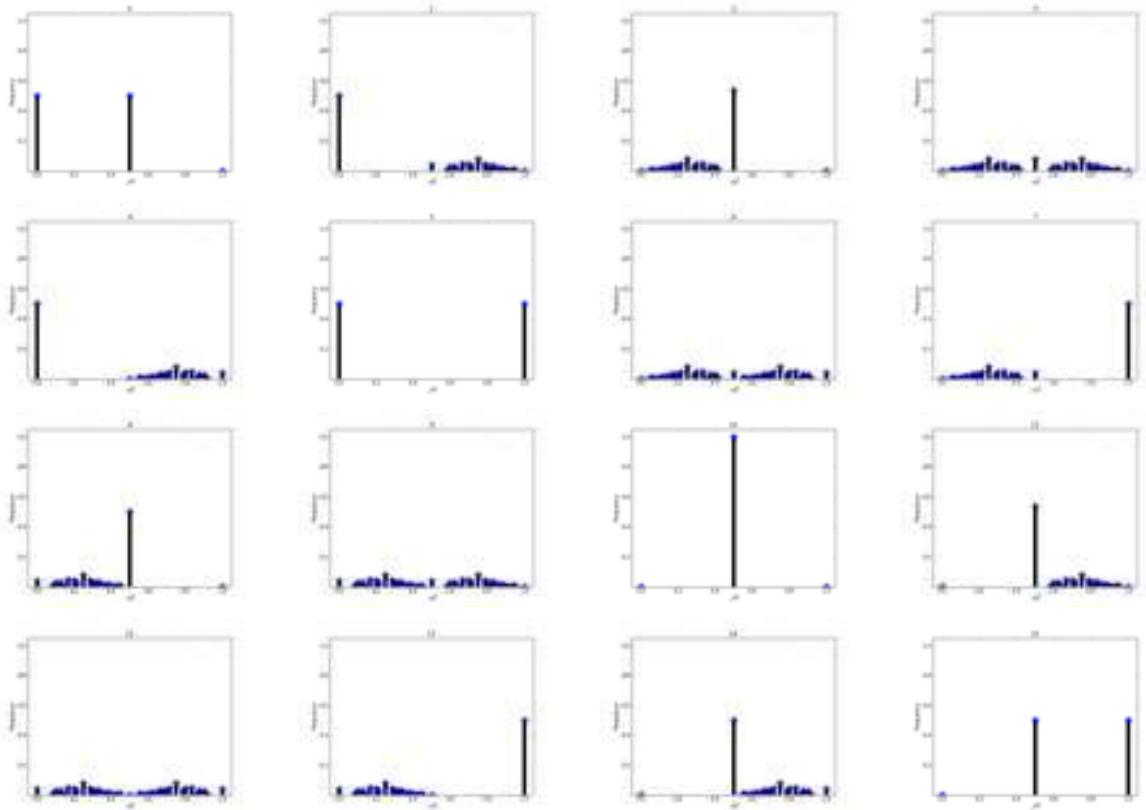

(C7) $\tilde{v}_{0}$ SL group
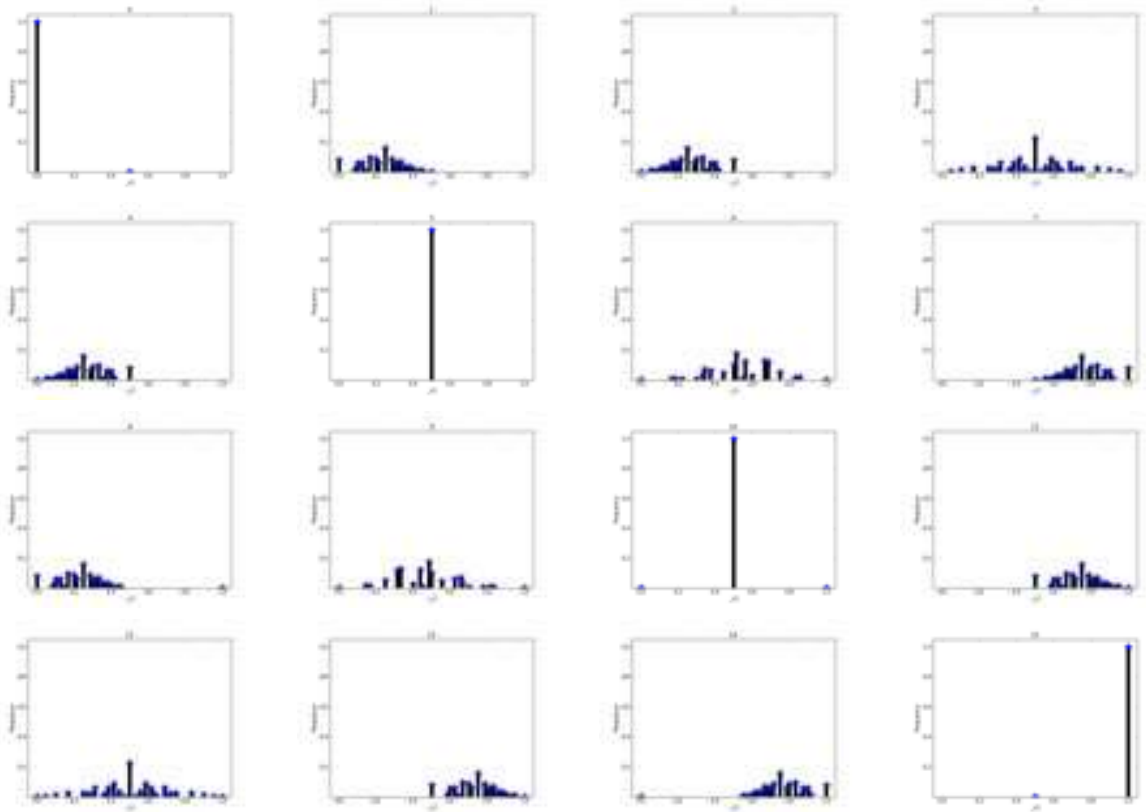

(C8) $\tilde{v}_{1}$ SL group 

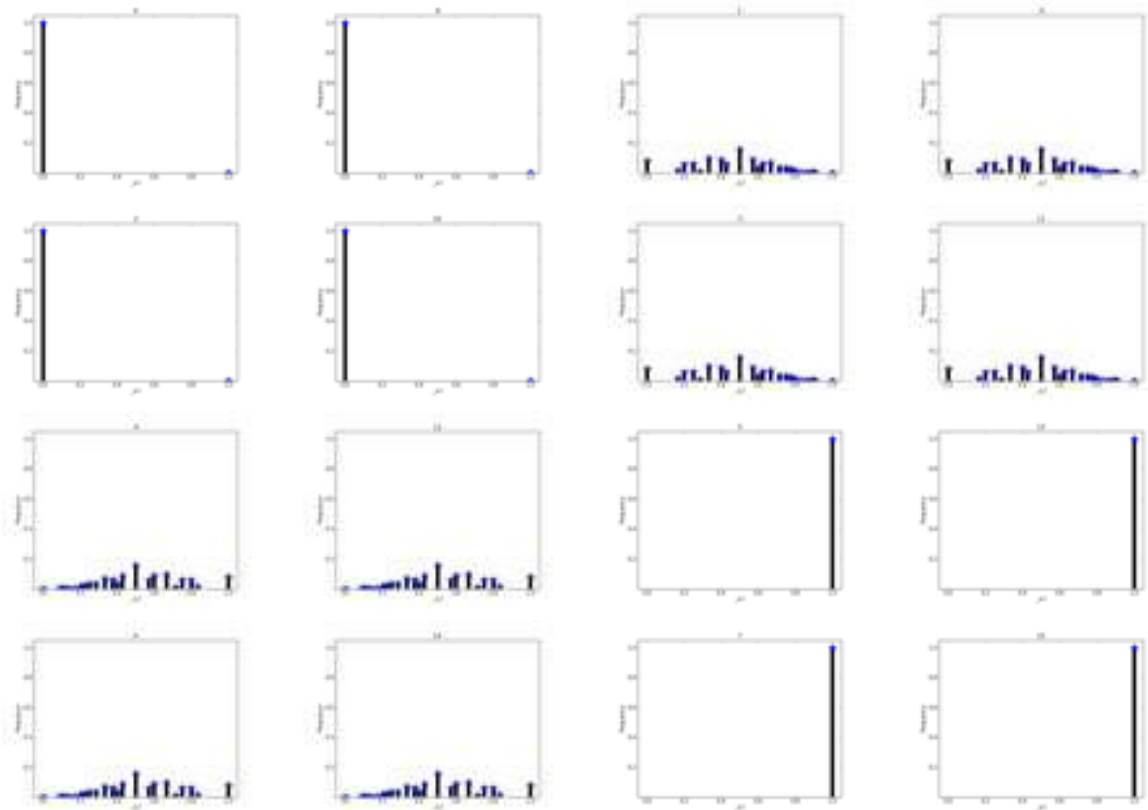

(C9) $\tilde{u}_{+} \mathrm{W}$ group
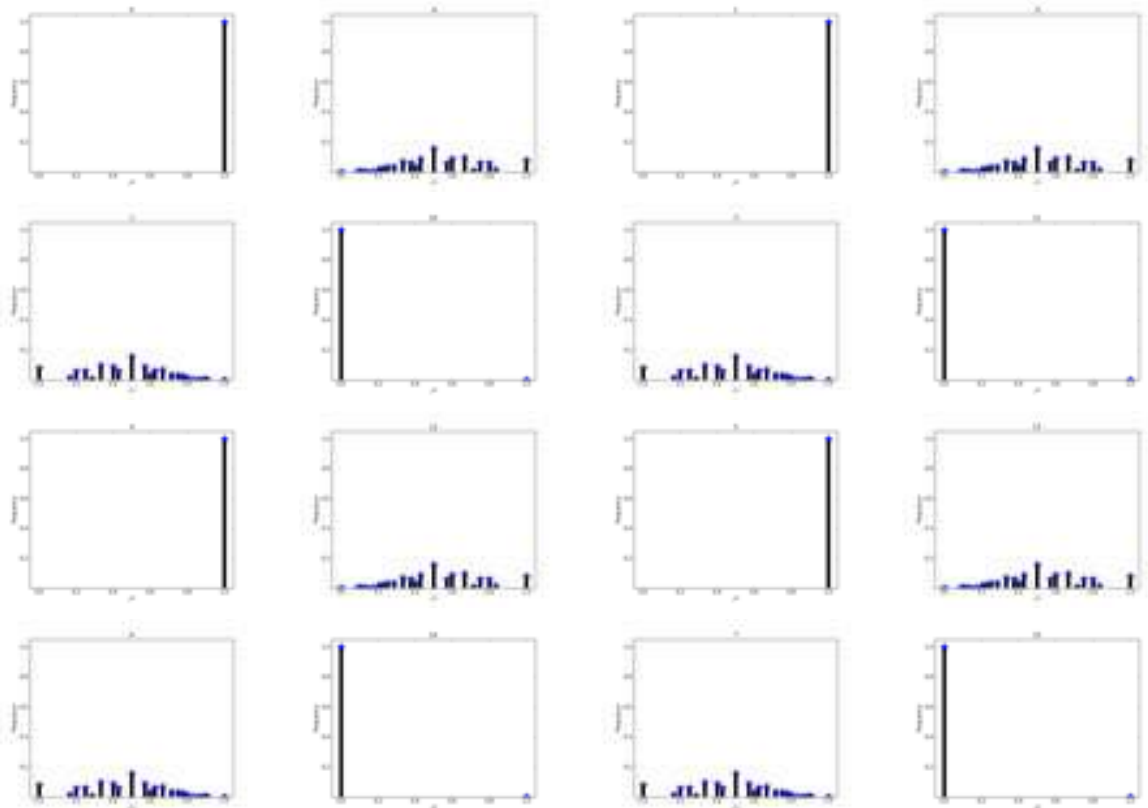

(C10) $\tilde{u}_{-}$W group 

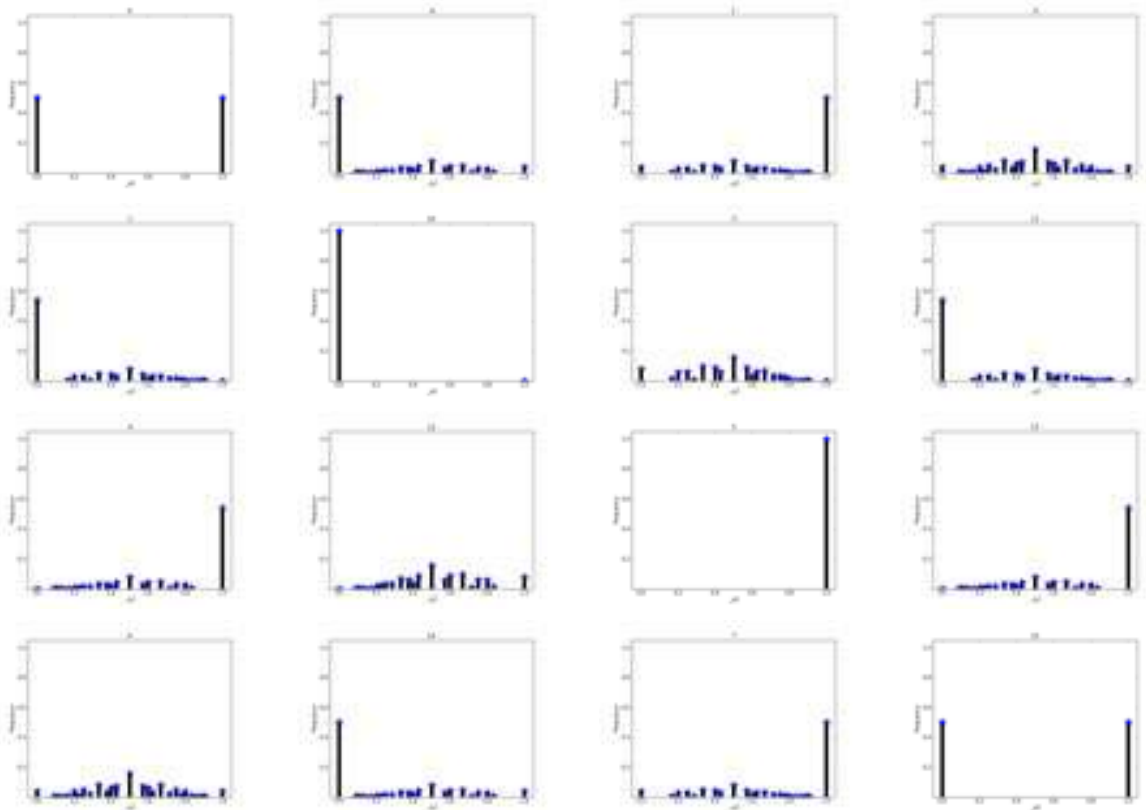

(C11) $\tilde{u}_{0} \mathrm{~W}$ group
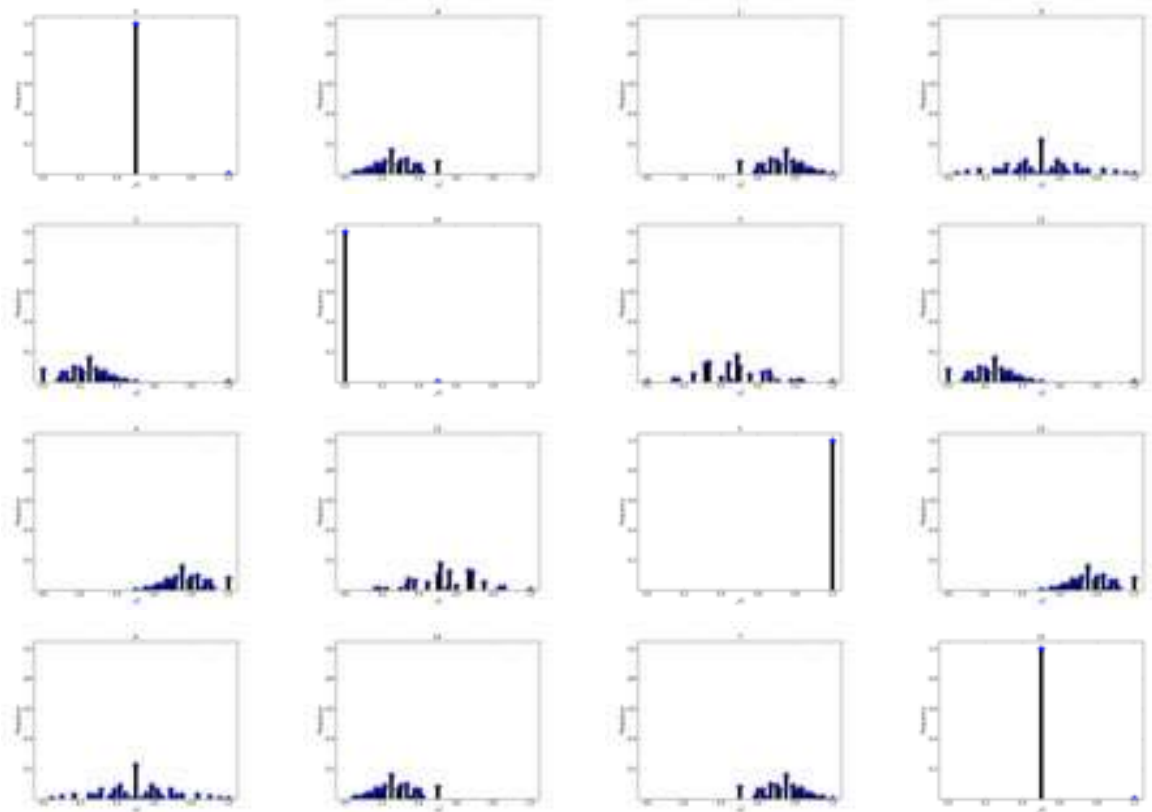

(C12) $\tilde{u}_{1} \mathrm{~W}$ group 

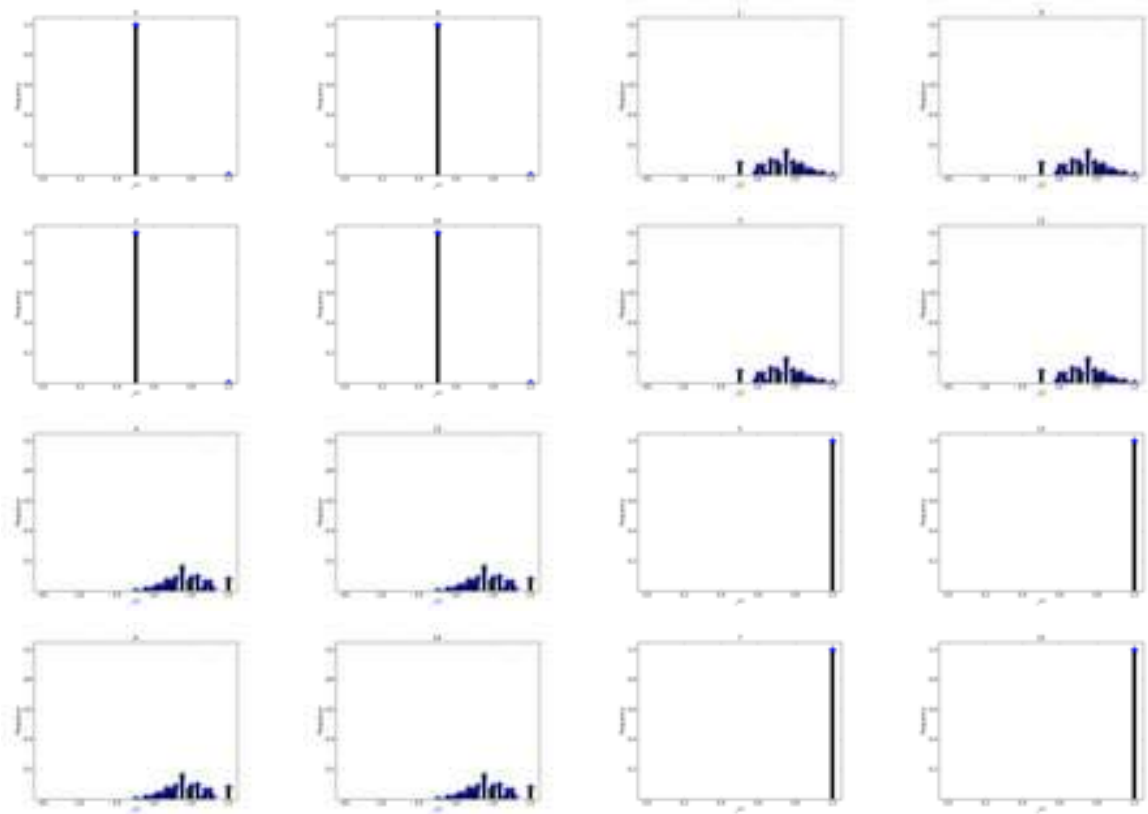

(C13) $\tilde{v}_{+} \mathrm{W}$ group
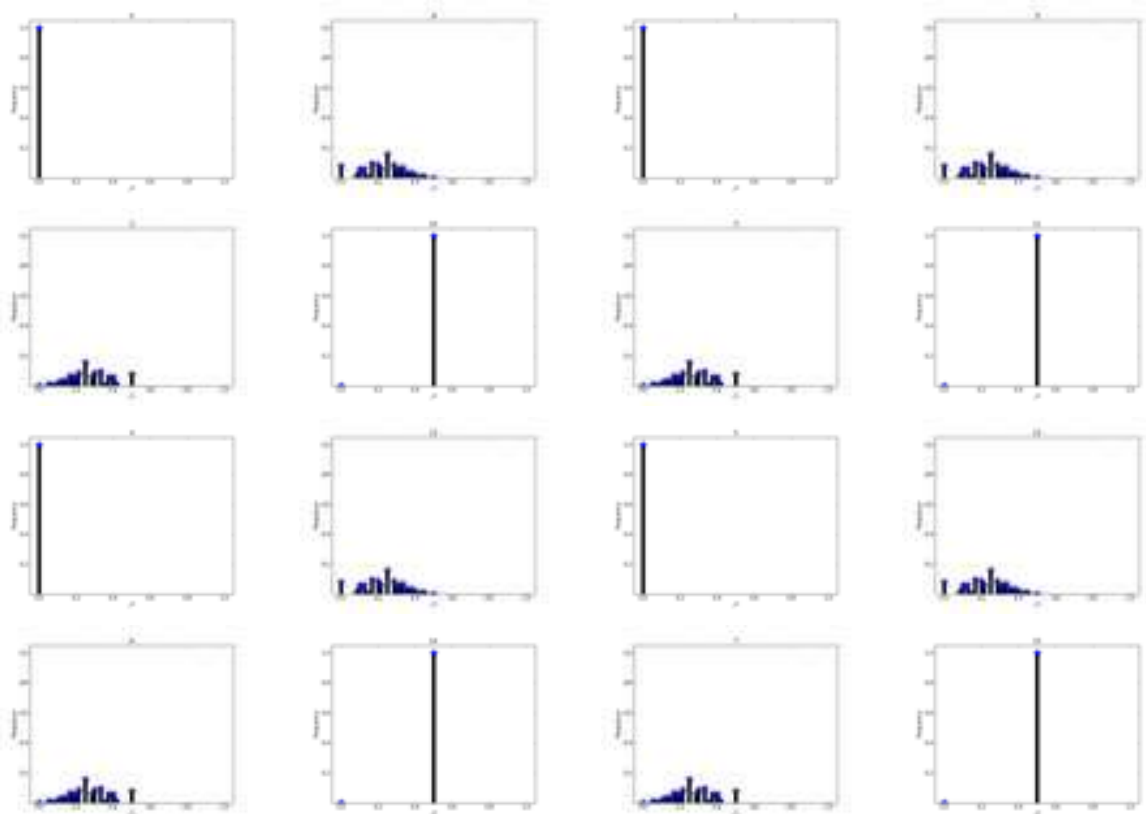

(C14) $\tilde{v}_{-}$W group 

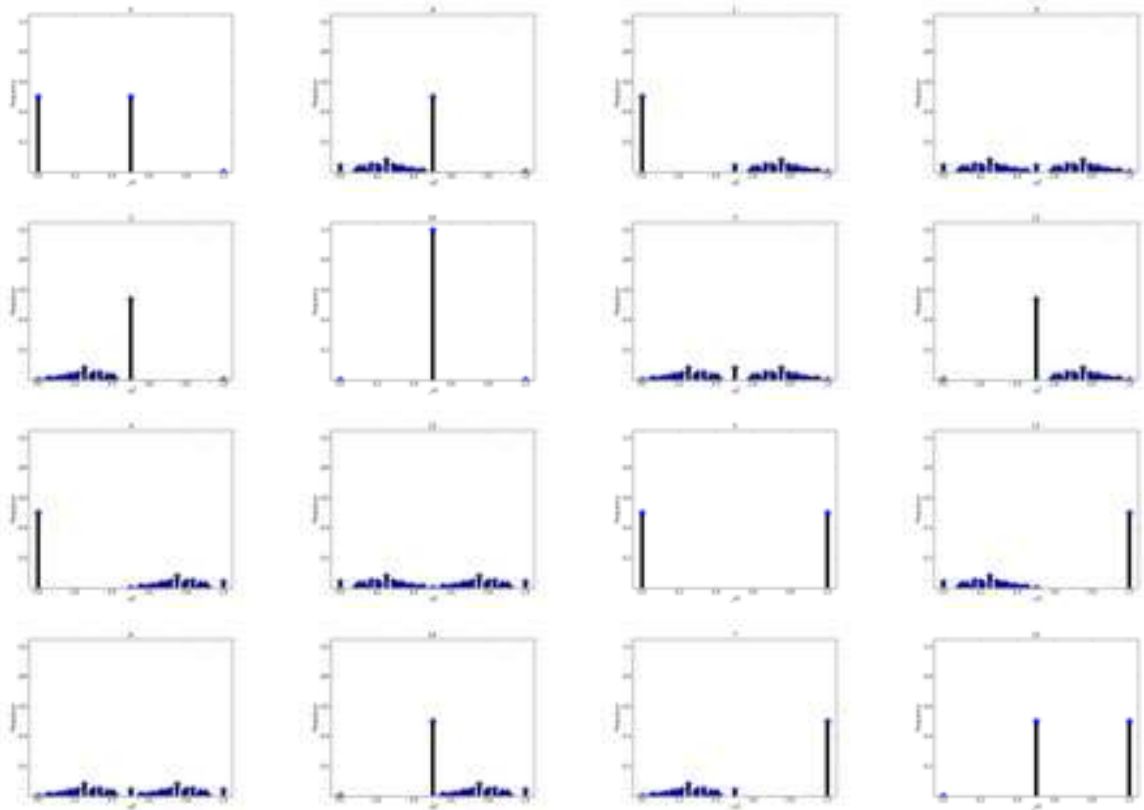

(C15) $\tilde{v}_{0} \mathrm{~W}$ group
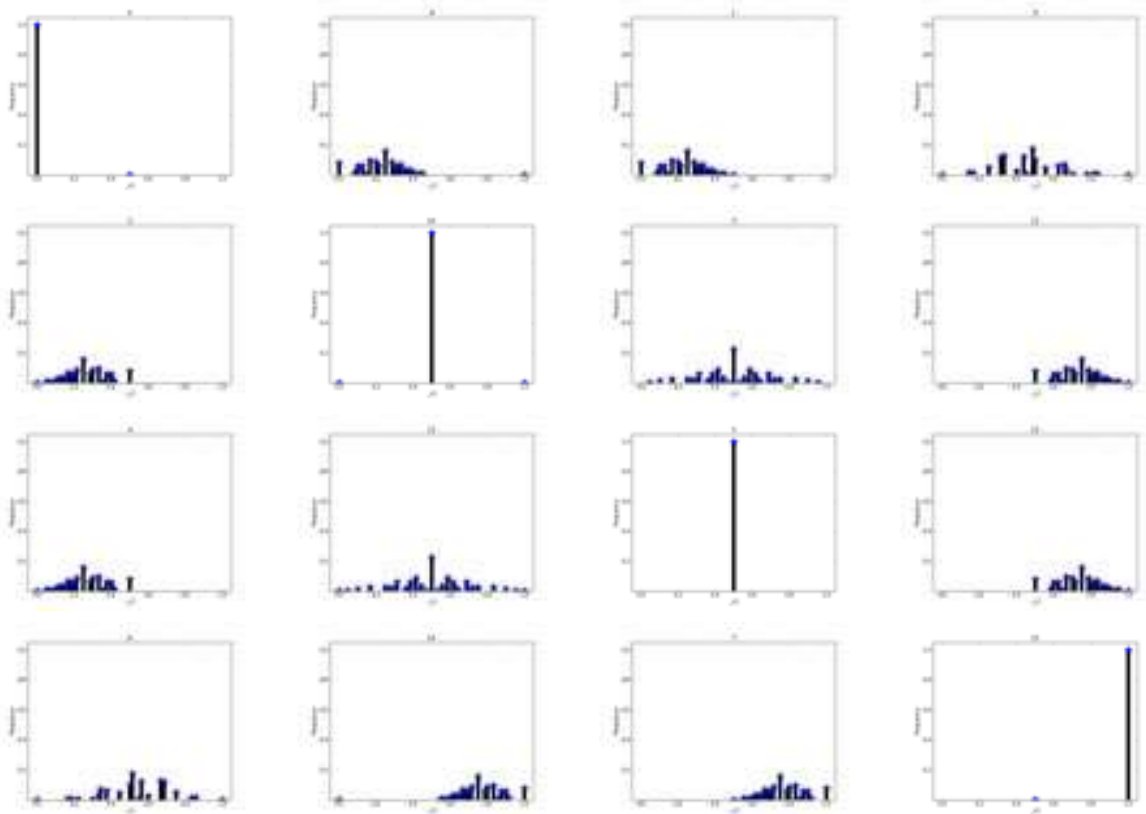

(C16) $\tilde{v}_{1} \mathrm{~W}$ group 

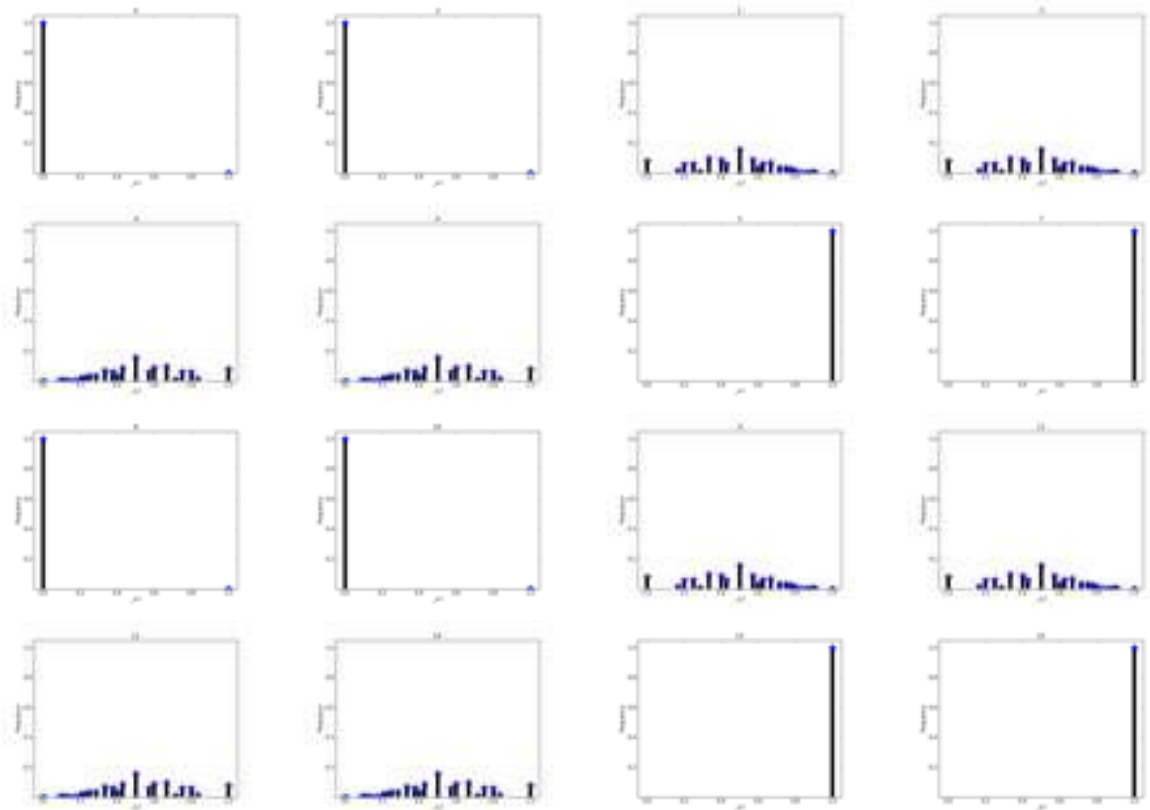

(C17) $\tilde{u}_{+}$F group
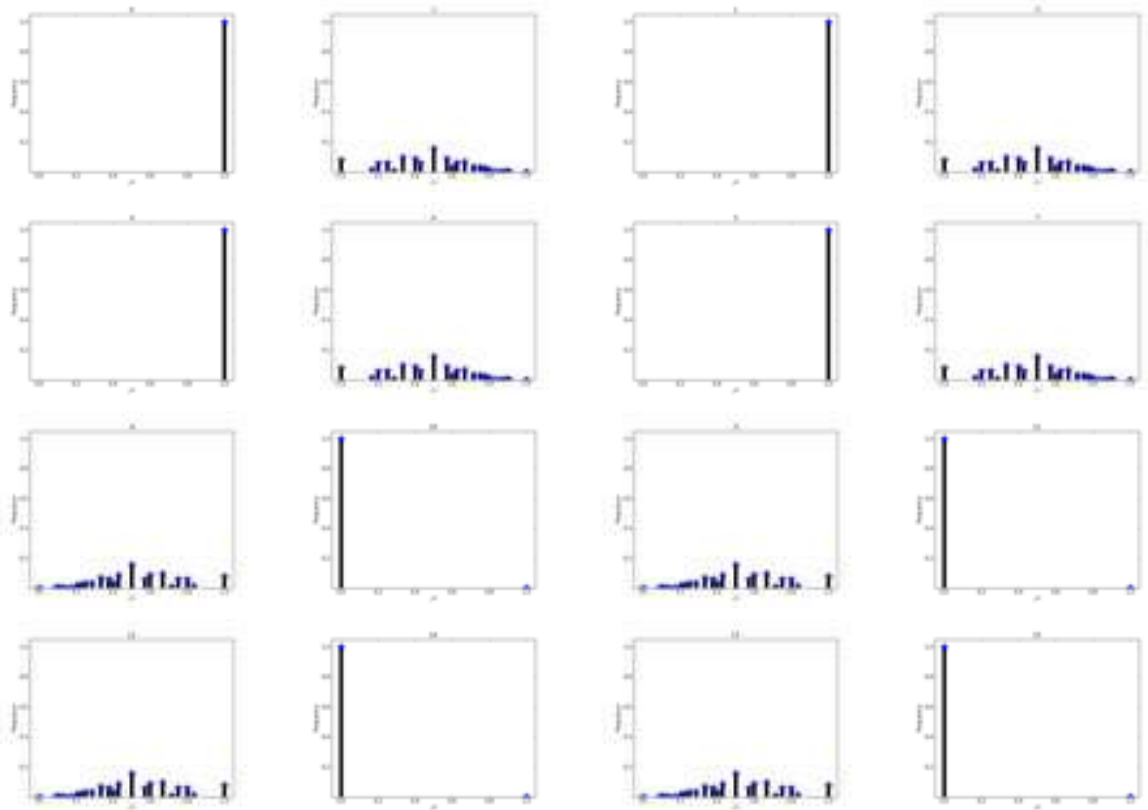

(C18) $\tilde{u}_{-}$F group 

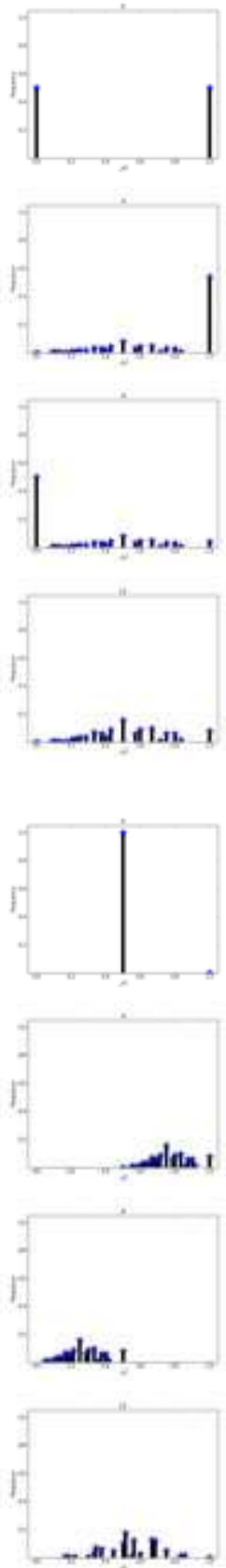
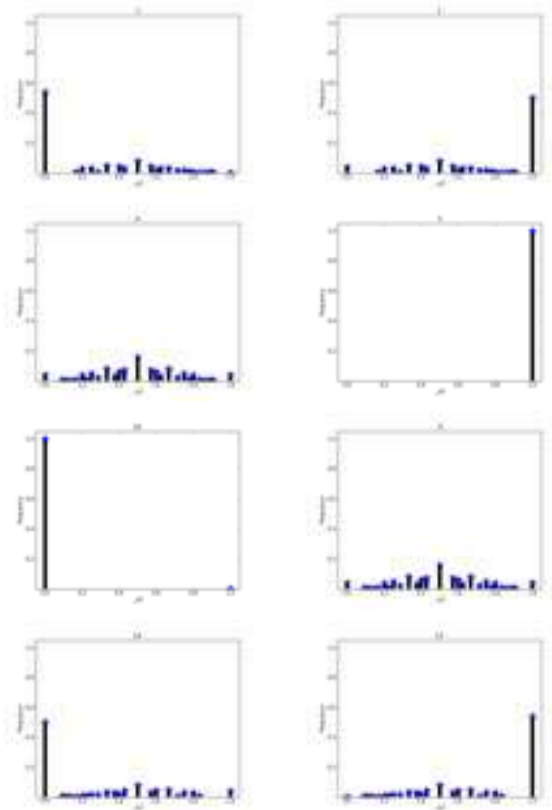

(C19) $\tilde{u}_{0}$ F group
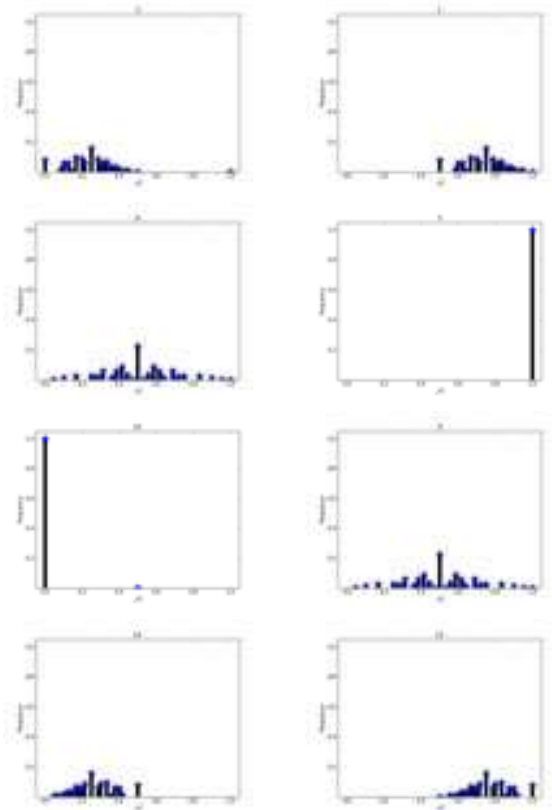

(C20) $\tilde{u}_{1}$ F group
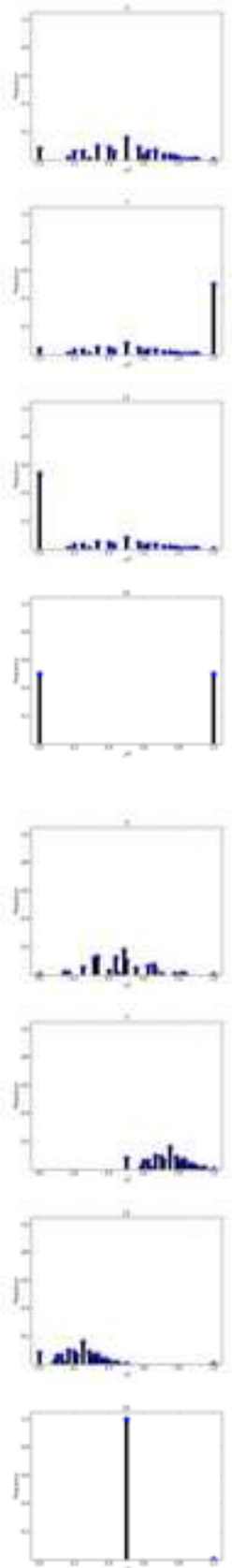

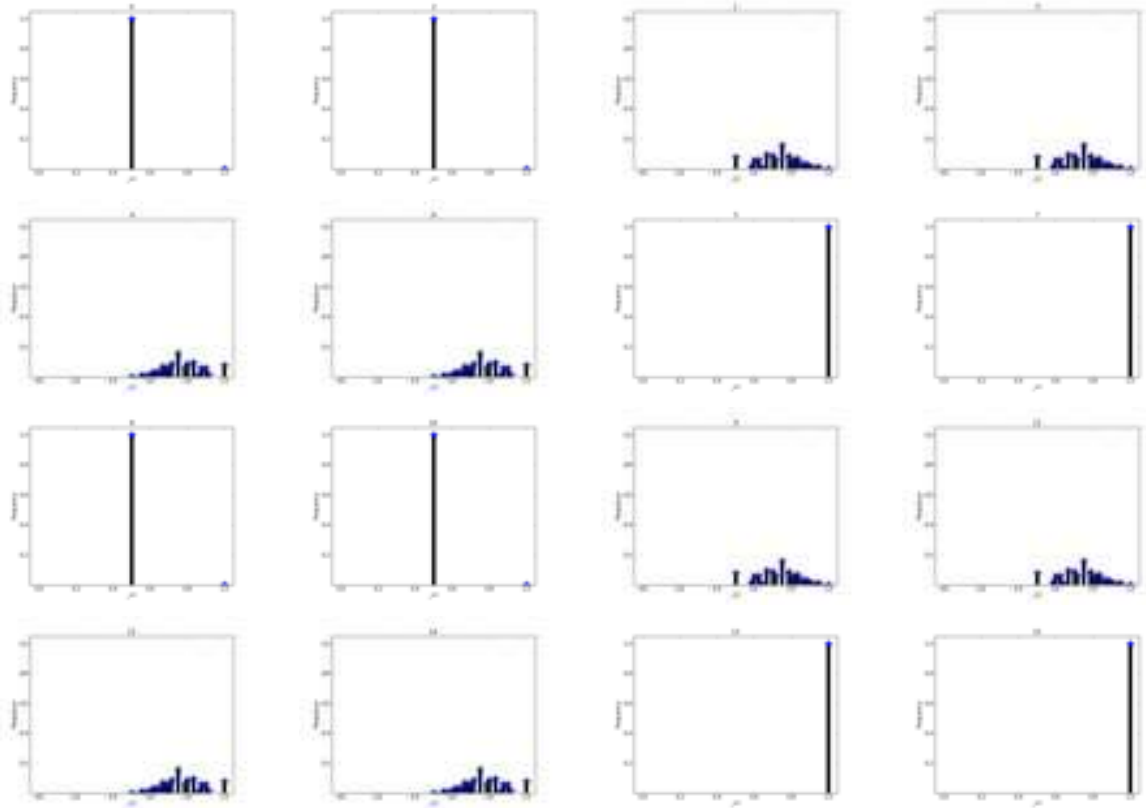

(C21) $\tilde{v}_{+}$F group
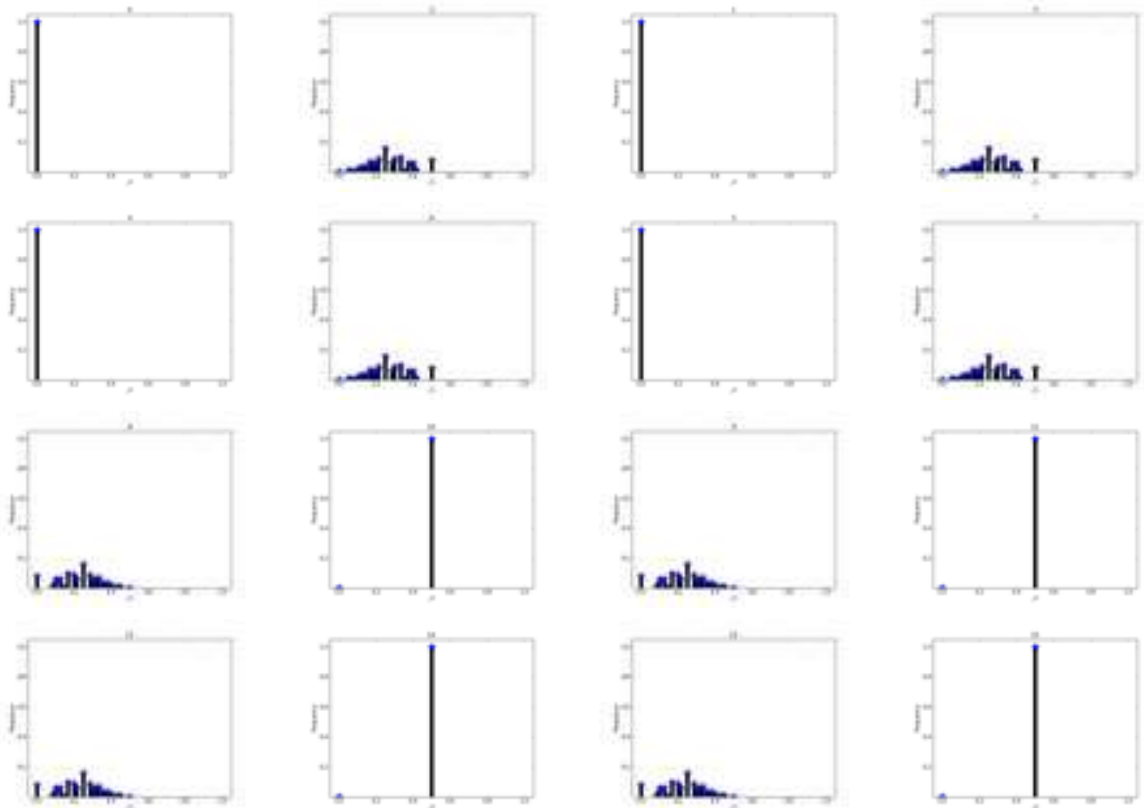

(C22) $\tilde{v}_{-}$F group 

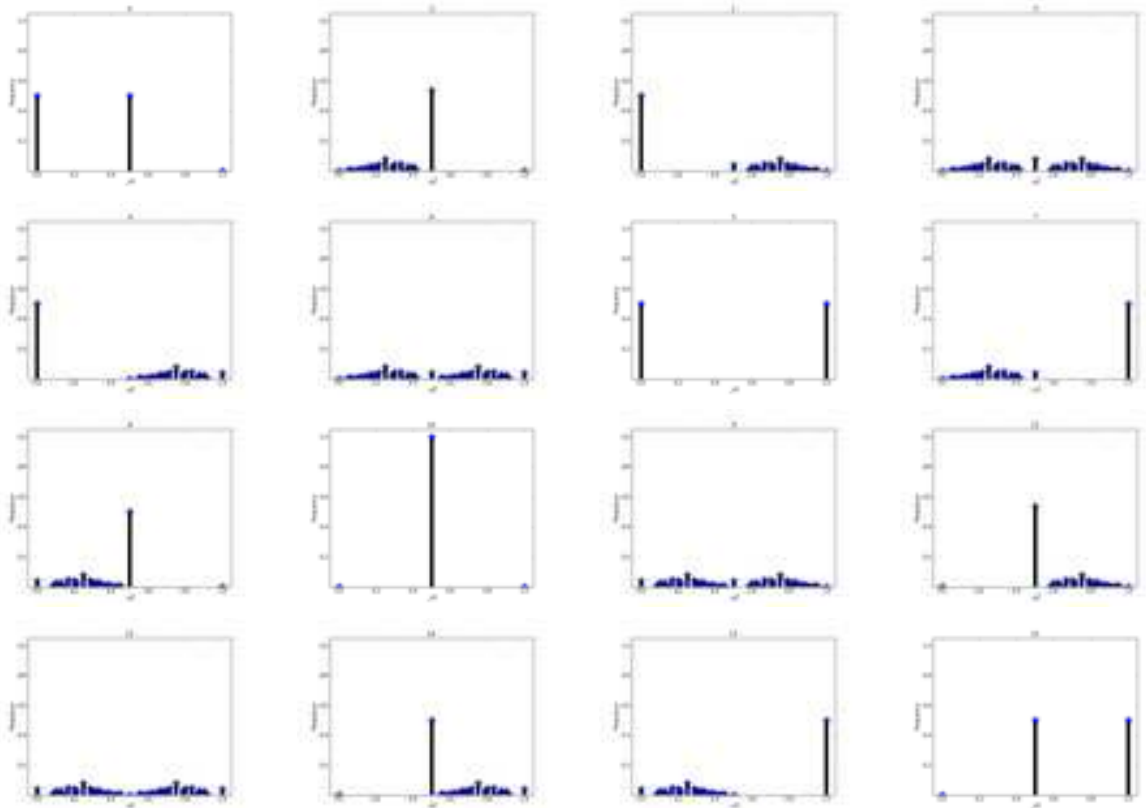

(C23) $\tilde{v}_{0}$ F group
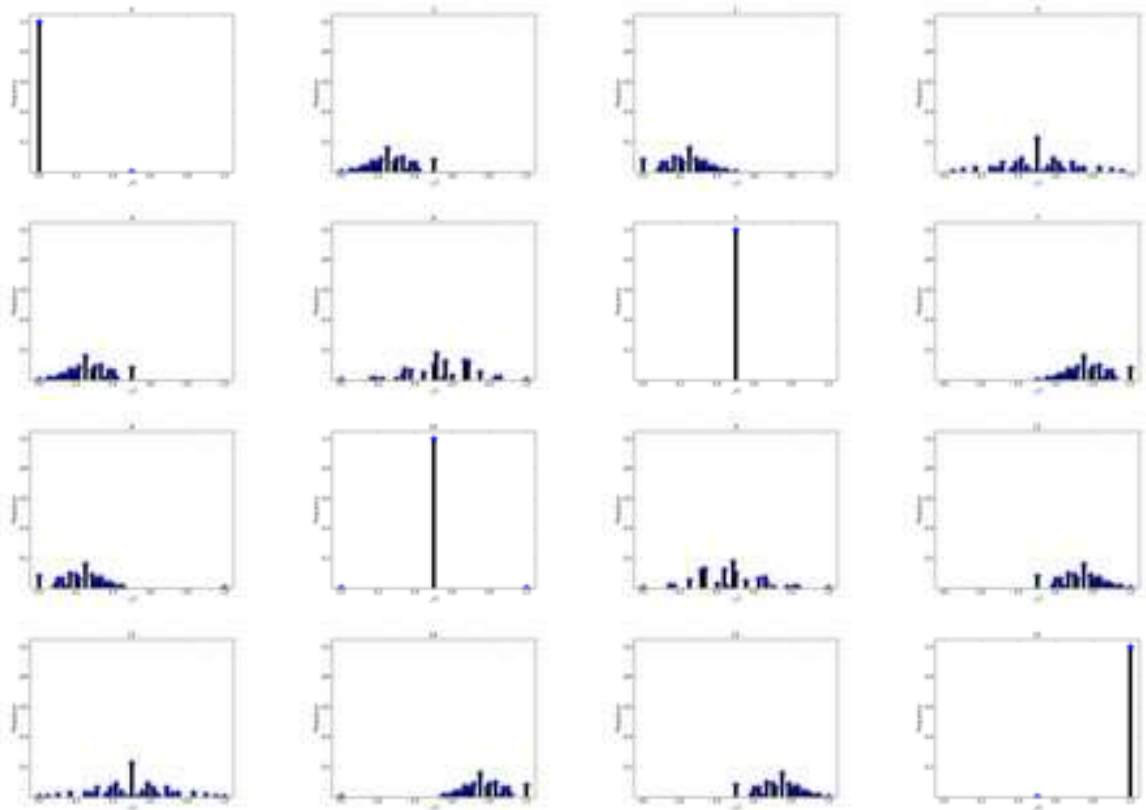

(C24) $\tilde{v}_{1}$ F group 

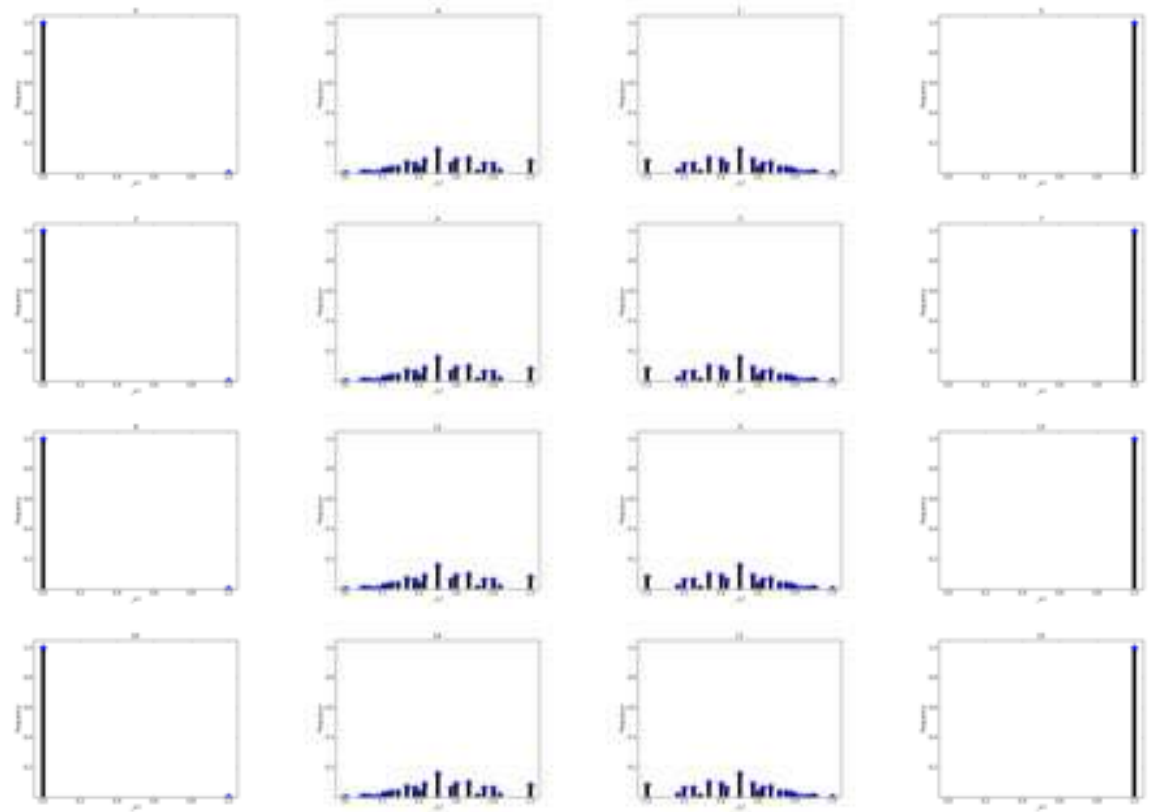

(C25) $\tilde{u}_{+}$C group
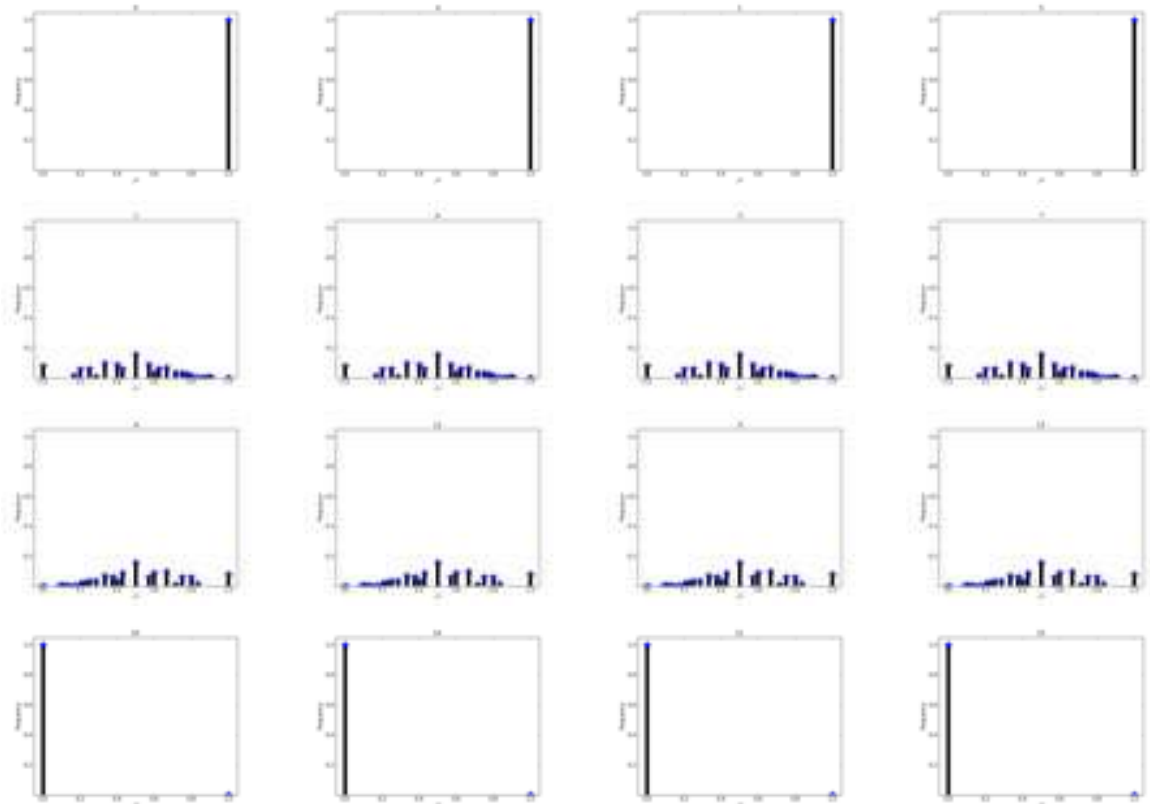

(C26) $\tilde{u}_{-}$C group 

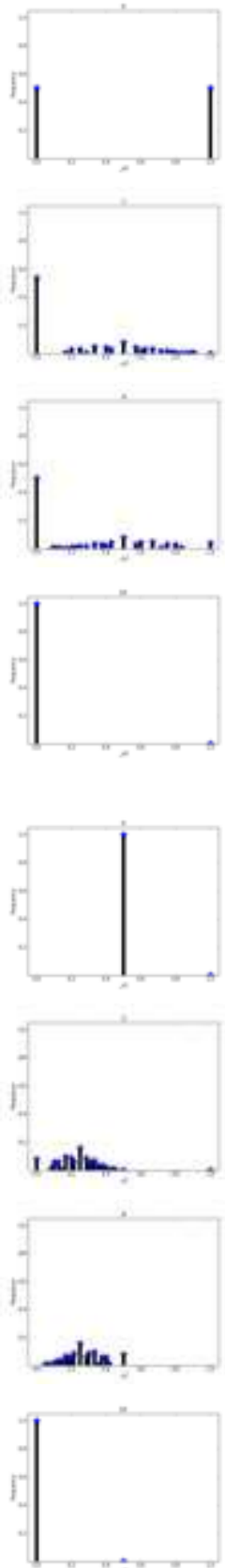
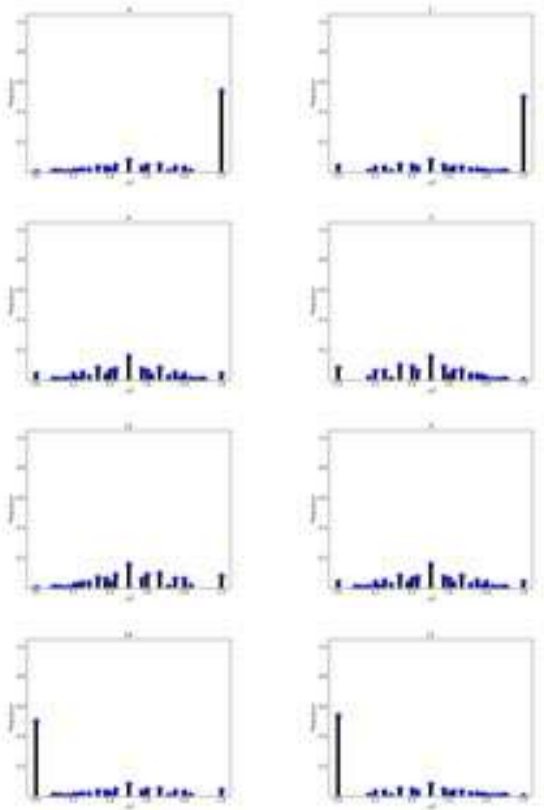

(C27) $\tilde{u}_{0} \mathrm{C}$ group
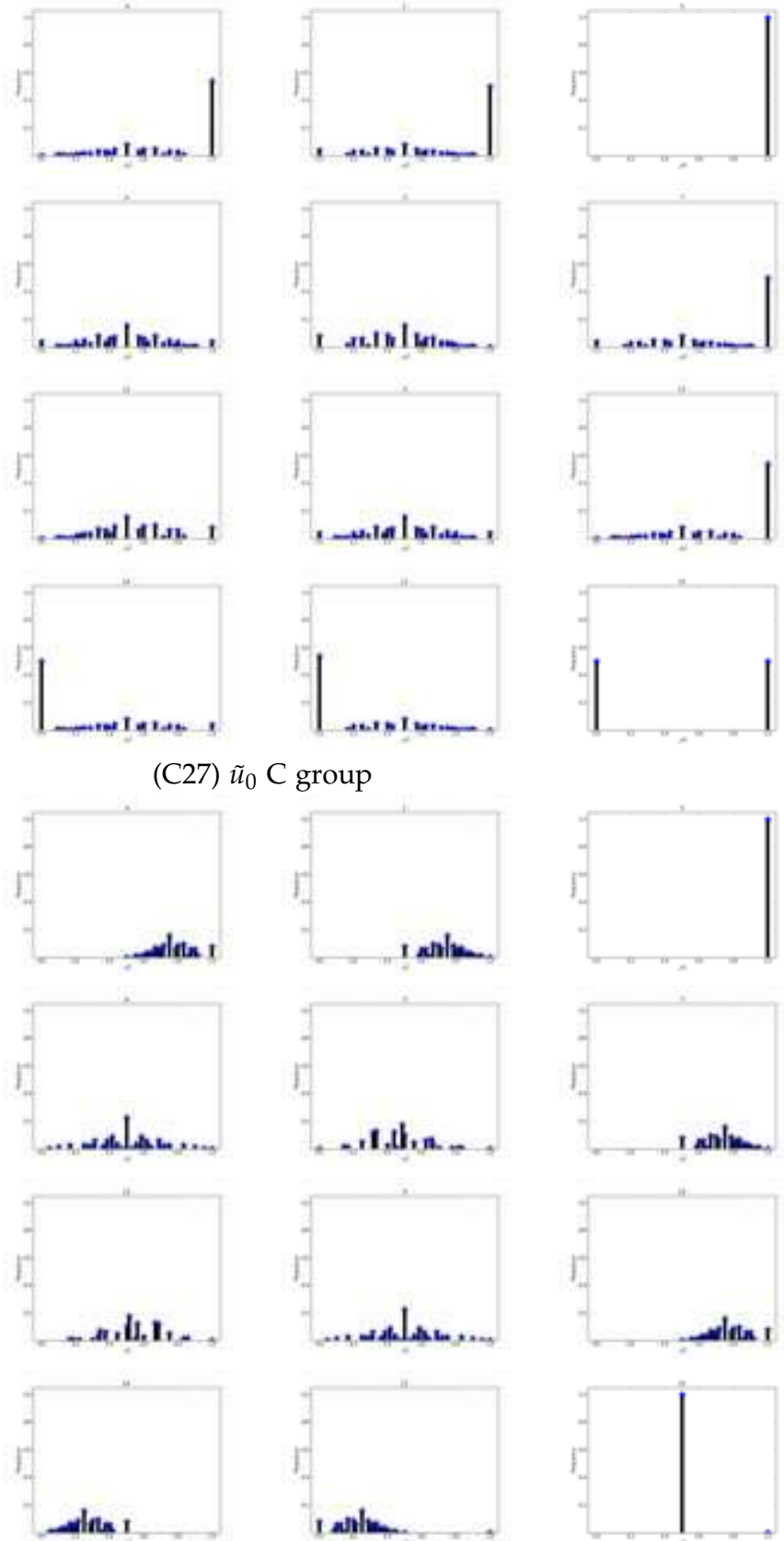

(C28) $\tilde{u}_{1} \mathrm{C}$ group 

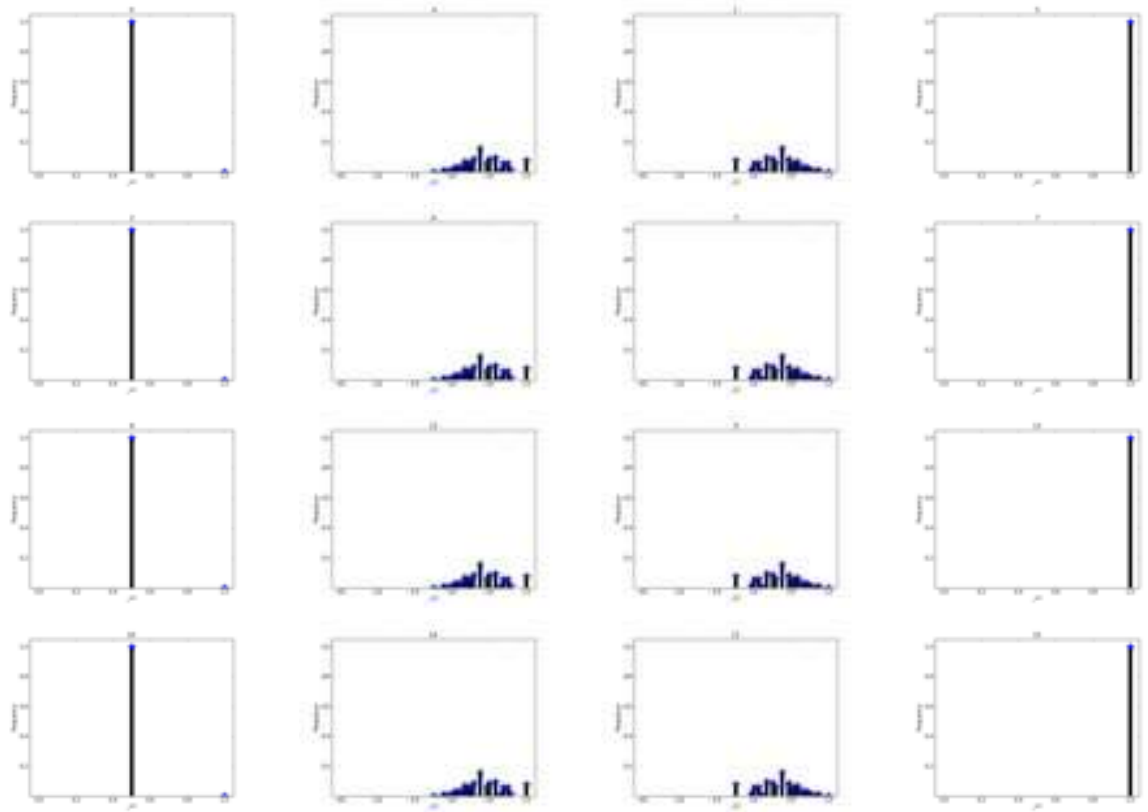

(C29) $\tilde{v}_{+}$C group
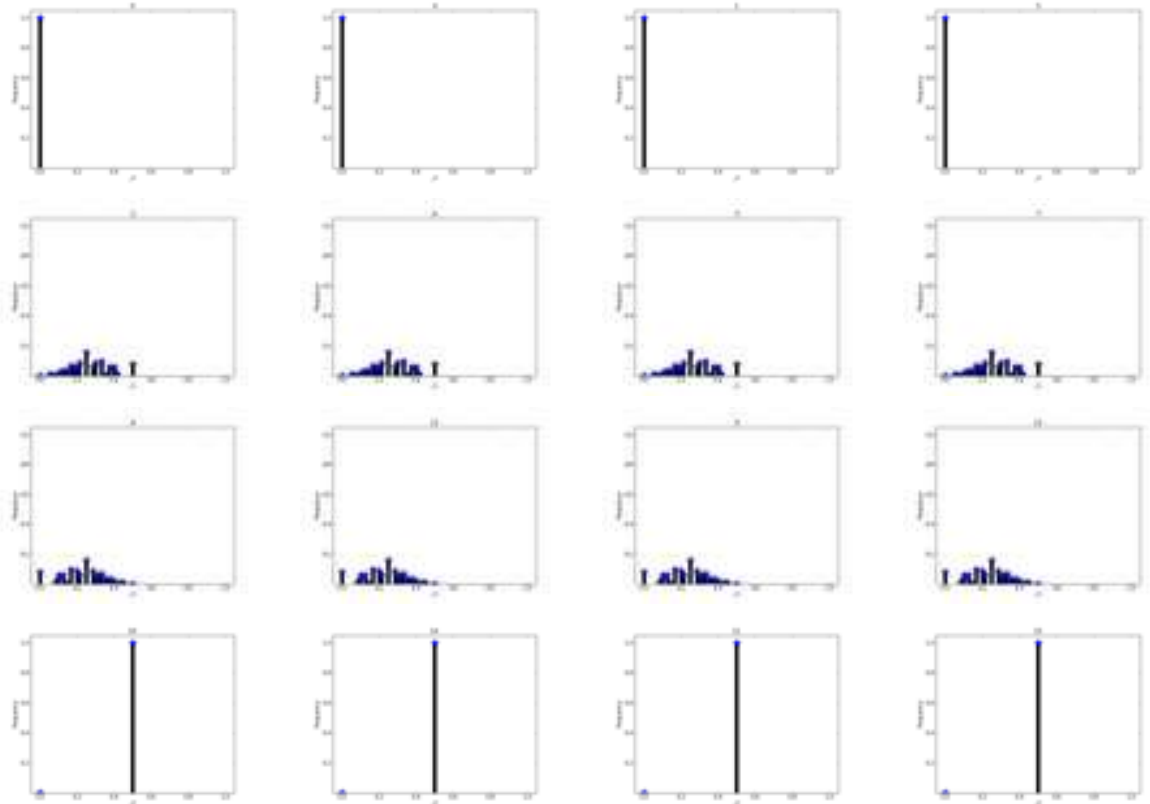

(C30) $\tilde{v}_{-}$C group 

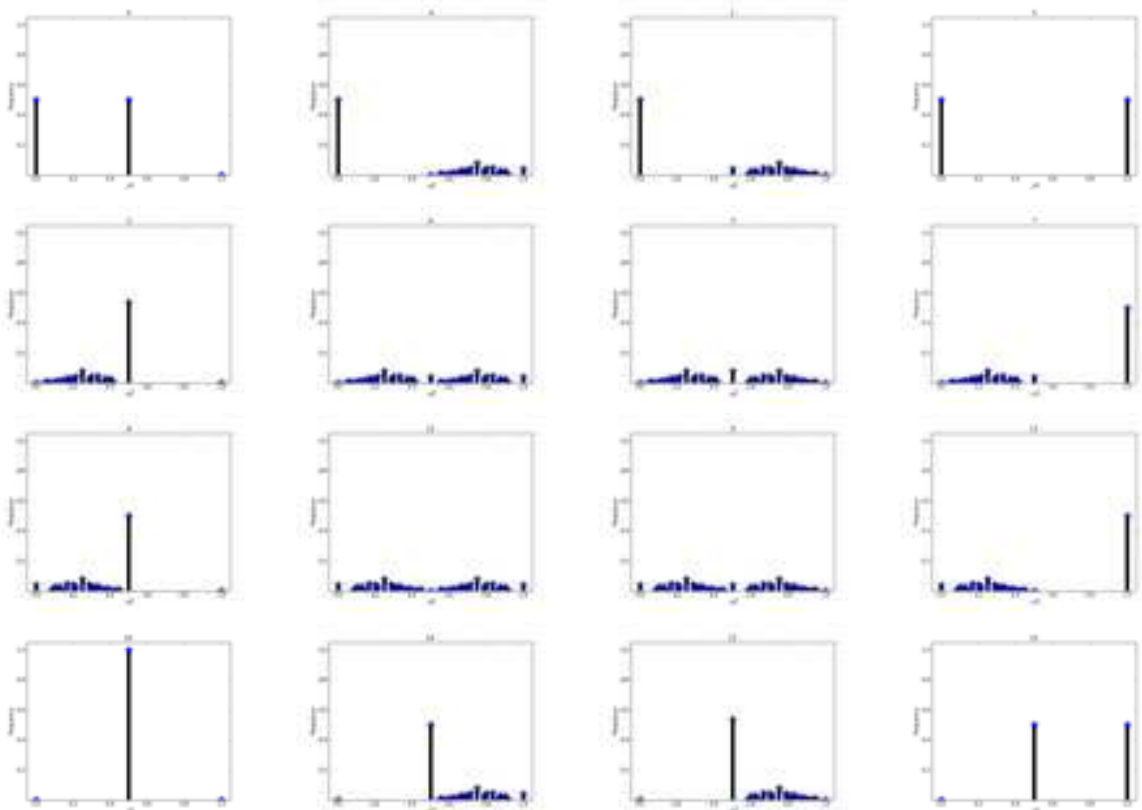

(C31) $\tilde{v}_{0} \mathrm{C}$ group
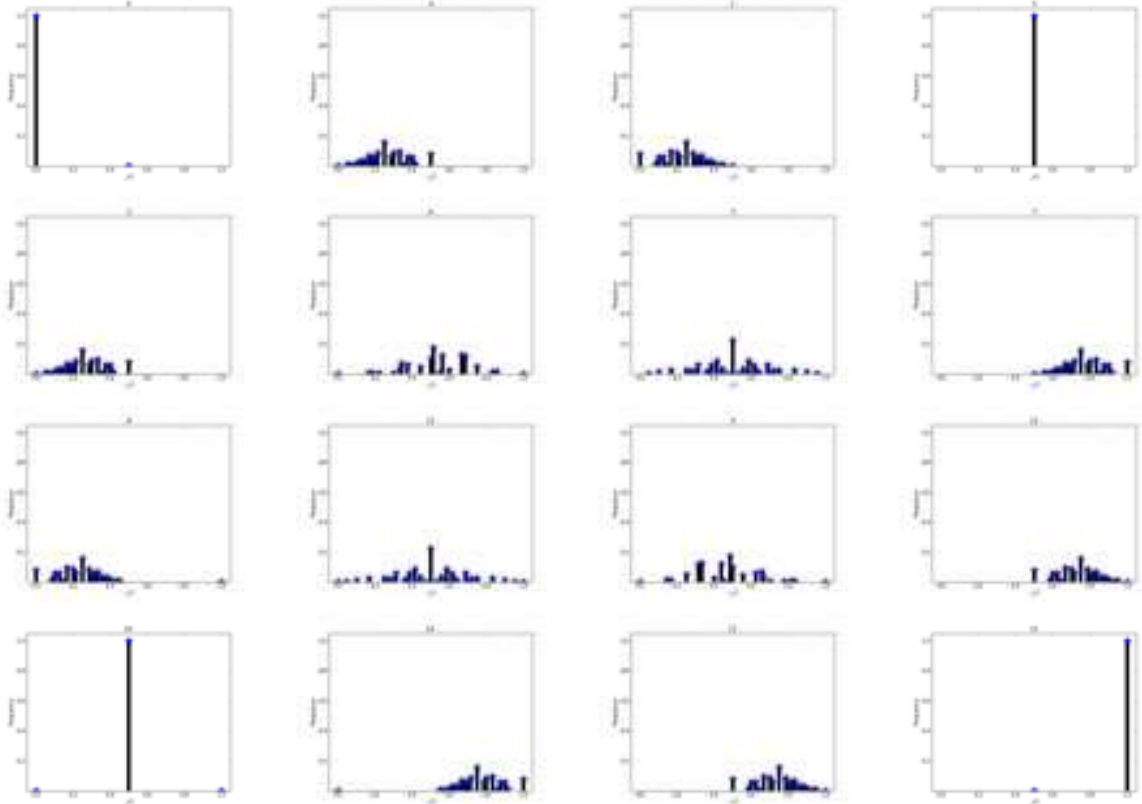

(C32) $\tilde{v}_{1} \mathrm{C}$ group

Figure 10. (C1-C32) IMM for CPS; (C1-C8) SL group; (C9-C16) W group; (C17-C24) F group; (C25-C32) C group. 


\section{Conclusion}

This chapter provides a brief investigation into Variant Phase Space (VPS) construction. Using an $n$ variable $0-1$ function and an $N$ bit vector, a VPS hierarchy can be progressively established via variant measures, multiple or conditional probability measurements, and selected pair of measurements to determine a Micro Ensemble (ME) and its eight interactive projections. Collecting all possible $2^{N}$ pairs of probability measurements, a Canonical Ensemble (CE) and its eight Interactive Maps (IMs) are generated following a bottom-up approach.

Applying a Maxwell demon mechanism, all possible $2^{2^{n}}$ functions can be calculated to create a result comprising a $\{\mathrm{CE}\}$ and eight sets of $\{\mathrm{IM}\}$. Using either a CE or an IM as an element, it is possible to use a variant logic configuration to organize each set of distributions to be a $2^{2^{n-1}} \times 2^{2^{n-1}}$ matrix as a CE Matrix (CEM) or IM Matrix (IMM), respectively. Following a top-down approach, a CEM or IMM can be decomposed into two polarized matrices with each matrix having periodic properties that meet the requirements of a Fourier-like transformation.

The main results are presented as ten propositions and four predictions to provide a foundation for further exploration of quantum interpretations, statistical mechanics, complex dynamic systems, and cellular automata.

The chapter does not explore global properties in detail, and further detailed investigations and expansions are necessary.

Anticipating that the principles put forward in this chapter will prove to be well founded, we look forward to exploring advanced scientific and technological applications in the near future.

\section{Acknowledgements}

Thanks to Professor Hui C. Shen of USTC for the selected works of de Broglie, and a historical review of statistical interpretation and modern development of statistical mechanics, to Colin W. Campbell for help with the English edition, to Jie Wan for MPS and CPS figures, to The School of Software Engineering, Yunnan University, The Key Laboratory of Software Engineering of Yunnan Province, and The Yunnan Advanced Overseas Scholar Project (W8110305) for financial support to the Information Security research projects (2010EI02, 2010KS06).

\section{Author details}

Jeffrey Zheng ${ }^{1}$, Christian Zheng $^{2}$ and Tosiyasu Kunii ${ }^{3}$

1 Yunnan University, Key Lab of Yunnan Software Engineering, P.R. China

2 University of Melbourne, Australia

3 University of Tokyo, Japan 


\section{References}

[1] Ash, R. B. \& Doléans-Dade, C. A. [2000]. Probability \& Measure Theory, Elsevier.

[2] Barrow, J. D., Davies, P. C. W. \& Charles L. Harper, J. E. [2004]. SCIENCE AND ULTIMATE REALITY: Quantum Theory, Cosmology and Complexity, Cambridge University Press.

[3] Belevitch V. [1962]. Summary of the history of circuit theory, Proceedings of the IRE, Vol 50, Iss $5,848-855$.

[4] Bender E.A. [2000]. An Introduction to Mathematical Modeling, Dover, New York.

[5] Birkhoff G.D. [1927]. Dynamic Systems, American Mathematical Society, New York.

[6] Blokhintsev D.I. [1964]. Quantum Mechanics, Dordrecht-Holland.

[7] Bohr, N. [1935]. Can quantum-mechanical description of physical reality be considered complete?, Physical Review 48. 696-702.

[8] Bohr, N. [1949]. Discussion with Einstein on Epistemological Problems in Atomic Physics, Evanston. 200-241.

[9] de Broglie, L.; Translated by Shen H.C. [2012]. Selected Works of de Broglie. in Chinese, Peijing University Press.

[10] Einstein, A., Podolsky, B. \& Rosen, N. [1935]. Can quantum-mechanical description of physical reality be considered complete?, Physical Review 47. 770-780.

[11] Feynman, R. [1965]. The Character of Physical Law, MIT Press.

[12] Feynman, R., Leighton, R. \& Sands, M. [1965,1989]. The Feynman Lectures on Physics, Vol. 3, Addison-Wesley, Reading, Mass.

[13] Gershenfeld N. [1998]. The Nature of Mathematical Modeling, Cambridge Uni. Press.

[14] Gibbs J.W. [1902]. Elementary Principles in Statistical Mechanics, Yale Uni. Press, New Haven.

[15] Goodwin G.C. and Payne R.L. [1977]. Dynamic System Identification:Experiment Design and Data Analysis, Academic Press.

[16] Healey, R., Hellman, G. \& Edited. [1998]. Quantum Measurement: Beyond Paradox, Uni. Minnesota Press.

[17] Hume J.N.P. [1974]. Physics in two volums, Vol. 2 Relativity, Electromagnetism and Quantum Physics, The Ronald Press Company, New York.

[18] Ivey D.G. [1974]. Physics in two volums, Vol. 1 Classical Mechanics and Introductory Statistical Mechanics, The Ronald Press Company, New York.

[19] Jammer, M. [1974]. The Philosophy of Quantum Mechanics, Wiley-Interscience Publication. 
[20] Khinchin A.J. [1949]. Mathematical Foundations of Statistical Mechanics, Dover, New York.

[21] Kurth R. [1960]. Axiomatics of Classical Statistical Mechanics, Pergamon Press, Oxford.

[22] Kuzemsky A.L. [2008]. Works by D.I. Blokhintsev and the Development of Quantum Physics, Physics of Partcles and Nuclei, Vol 39, No.2 137-172. DOI:10.1134/S1063779608020019

[23] Landau L.D. and Lifshitz E.M. [1996]. Statistical Physics, 3rd Edition Part 1, Butterworth-Heinemann, Oxford.

[24] Lee Tsung-Dao [2006]. Statistical Mechanics, in Chinese, Shanghai Science and Technology Press.

[25] Nelles O. [2001]. Nonlinear System Identification, Springer.

[26] Nolte D.D. [2010]. The tangled tale of phase space. Physics Today, April 2010. 33-39. http://www.physicstoday.org

[27] Penrose, R. [2004]. The Road to Reality, Vintage Books, London.

[28] Pintelon P. and Schoukens J. [2001]. System Identification: A frequency domain approach, IEEE Press, New York.

[29] Pring M.J. [2002]. Breaking the Black Box, McGraw-Hill.

[30] Reif F. [1967]. Statistical Physics, Berkley Physics Course - Vol. 5, McGraw-Hill.

[31] Shen Hui-Chuan [2011]. Statistical Mechanics, in Chinese, University of Science and Technology of China Press.

[32] von Neumann, J. [1932,1996]. Mathematical Foundations of Quantum Mechanics, Princeton Univ. Press.

[33] Wu, Ta-You [2010]. Thermodynamics, Gas-dynamics and Statistical Mechanics, in Chinese, Scientific Press, Beijing.

[34] Zeh, H. D. [1970]. On the interpretation of measurement in quantum theory, Foundation of Physics 1. 69-76.

[35] Zheng, J., Zheng, C. \& Kunii T. [2012]. From Local Interactive Measurements to Global Matrix Representations on Variant Construction ÂĺC A Particle Model of Quantum Interactions for Double Path Experiments, Advanced Topics in Measurements, edited by: Z. Haq 371-400.

URL: http://www.intechopen.com/books/advanced-topics-in-measurements

[36] Zheng, J., Zheng, C. \& Kunii T. [2012]. From Conditional Probability Measurements to Global Matrix Representations on Variant Construction ÂíC A Particle Model of Intrinsic Quantum Waves for Double Path Experiments, Advanced Topics in Measurements, edited by: Z. Haq 337-370.

URL: http://www.intechopen.com/books/advanced-topics-in-measurements 
[37] Zheng, J. [2012]. Multiple and Conditional Probabilities and Their Statistical Distributions for Variant Measures, Laser E Optoelectronics Progress 49(4): 042701.

URL: http://www.opticsjournal.net/abstract.htm?aid=OJ120119000021MjPlSo

[38] Zheng, J. [2011]. Synchronous properties in quantum interferences, Journal of Computations \& Modelling, International Scientific Press 1(1). 73-90.

URL: http://www.scienpress.com/upload/JCM/Vol\%201_1_6.pdf

[39] Zheng, J. \& Zheng, C. [2011]. Variant measures and visualized statistical distributions, Acta Photonica Sinica, Science Press 40(9). 1397-1404. URL: http://www.photon.ac.cn/CN /article/downloadArticleFile.do?attachType=PDFEid=15668

[40] Zheng, J., Zheng, C. \& Kunii, T. [2011]. A framework of variant-logic construction for cellular automata, Cellular Automata - Innovative Modelling for Science and Engineering edited Dr. A. Salcido, InTech Press. 325-352. URL: http://www.intechopen. com/articles/show/title/a-framework-of-variant-logic-construction-for-cellular-automata

[41] Zheng, J. [2011]. Conditional Probability Statistical Distributions in Variant Measurement Simulations, Acta Photonica Sinica, Science Press 40(11): 1662-1666.

URL: http://www.opticsjournal.net/viewFull.htm?aid=OJ1112120000332y5B8D

[42] Zheng, J. \& Zheng, C. [2011]. Variant simulation system using quaternion structures, Journal of Modern Optics, Taylor \& Francis Group 59(5): 484-492.

URL: http://www.tandfonline.com/doi/abs/10.1080/09500340.2011.636152

[43] Zheng, J. [2011]. Two Dimensional Symmetry Properties of GlobalCoding Family on Configuration Function Spacesof Variant Logic, Journal of Chengdu University of Information Technology Dec. 2011.

URL: http://www.cnki.net/kcms/detail/51.1625.TN.20111227.1043.001.html

[44] Zheng, J. \& Zheng, C. [2010]. A framework to express variant and invariant functional spaces for binary logic, Frontiers of Electrical and Electronic Engineering in China, Higher Education Press and Springer 5(2): 163-172.

URL: http://www.springerlink.com/content/91474403127n446u/ 